\title{
An Option Pricing Formula for the GARCH Diffusion Model
}

\author{
Claudia Ravanelli \\ Submitted for the degree of Ph.D. in Economics \\ University of Lugano, Switzerland \\ Prof. G. Barone-Adesi, University of Lugano, advisor \\ Prof. M. Chesney, University of Zurich \\ Prof. P. Vanini, University of Lugano
}

March 2004 
To my darling mom Silvana 


\section{Acknowledgments}

It is a great pleasure to thank Professor Giovanni Barone-Adesi, my thesis advisor, for invaluable suggestions and discussions and for his support during the last three years.

I am grateful to Professor Paolo Vanini for his useful comments and suggestions and for accepting to be a member of the thesis committee.

I really wish to thank Professor Marc Chesney for accepting to be co-examiner of this work.

Thanks go to my friends Francesca Bellini, Ettore Croci, Silvia Cappa, Talia Cicognani, Clizia Lonati and Silvia Zerbeloni for making everyday life enjoyable.

My gratitude to my mom Silvana and to my brother Renato for their continuous support during these years.

Specially thanks go to Loriano for his love and support and for always enriching my life. 


\section{Contents}

$\begin{array}{ll}\text { Acknowledgments } & \text { ii }\end{array}$

$\begin{array}{ll}\text { Introduction } & 1\end{array}$

1 Stochastic Volatility Models $\quad 6$

1.1 Motivations of Stochastic Volatilities . . . . . . . . . . . . . . . 6

1.2 The GARCH Diffusion Model . . . . . . . . . . . . . . . . . . . . . . . . . . 10

2 Option Pricing under the GARCH Diffusion Model $\quad 14$

2.1 The Option Pricing Formula . . . . . . . . . . . . . . . . . . . . . 14

2.2 Monte Carlo Simulations . . . . . . . . . . . . . . . . . . . . . . . . . . . 20

2.3 Effects of Stochastic Volatility on Option Prices . . . . . . . . . . . . . . . . . . . 24

2.4 Implied Volatility Surfaces . . . . . . . . . . . . . . . . . . . . . . . 25

3 Inference based on Nelson's Theory $\quad 40$

3.1 Simple Estimators for the GARCH Diffusion Model . . . . . . . . . . . . . . . . . . . . 41

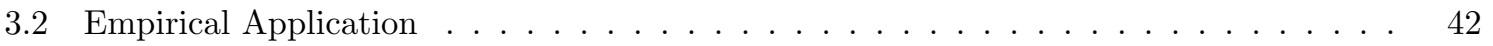

4 Conclusions $\quad 51$ 
A.1 Proof of Proposition $2.1 \ldots \ldots \ldots \ldots$

A.1.1 First conditional moment . . . . . . . . . . . . . . . . . . . . . . . . 54

A.1.2 Second conditional moment . . . . . . . . . . . . . . . . 55

A.2 Conditional Moments of $\bar{V}_{T}$ under Log-Normal Variance . . . . . . . . . . . . . . . . 58

A.3 The Market Price of Risk and the Novikov's Condition . . . . . . . . . . . . . . . . . . 61

A.4 Nelson's Moment Matching Procedure . . . . . . . . . . . . . . . . . . . . . . . . . . 63 


\section{List of Figures}

$2.1 c_{3}$ effect. Percent Bias $=100\left(\widetilde{C}^{g d}-C_{b s}\right)$ for different maturities and parameter values of $c_{3}$, when $S_{0}=100, r=d=0 ; d V=(0.09-4 V) d t+c_{3} V d W, V_{0}=0.0225 . \ldots . . . .$.

$2.2 c_{2}^{*}$ effect. Percent Bias $=100\left(\widetilde{C}^{g d}-C_{b s}\right)$ for different maturities and parameter values of $c_{2}^{*}$, when $S_{0}=100, r=d=0 ; d V=\left(0.9-c_{2}^{*} V\right) d t+1.2 V d W, V_{0}=0.0225 \ldots . . . . .$.

$2.3 c_{1}$ effect. Percent Bias $=100\left(\widetilde{C}^{g d}-C_{b s}\right)$ for different maturities and parameter values of $c_{1}$, when $S_{0}=100, r=d=0 ; d V=\left(c_{1}-4 V\right) d t+1.2 V d W, V_{0}=0.0225 \ldots \ldots . . .$.

$2.4 V_{0}$ effect. Percent Bias $=100\left(\widetilde{C}^{g d}-C_{b s}\right)$ for different maturities and initial variance $V_{0}$, when $S_{0}=100, r=d=0 ; d V=(0.09-4 V) d t+1.2 V d W \ldots \ldots \ldots \ldots$

2.5 Volatility smiles for maturities of $30,60,90$ and 120 days and the parameter choice $S_{0}=100$, $r=d=0 ; d V=(0.09-4 V) d t+1.2 V d W, V_{0}=0.0225$, as in Table 2.5. . . . . . . . .

2.6 Volatility Surface for maturities $T \in[30,120]$ days, strikes $K \in[90,110]$ and the parameter choice $S_{0}=100, r=d=0 ; d V=(0.09-4 V) d t+1.2 V d W, V_{0}=0.0225$, as in Table 2.5. $\quad 38$

2.7 Relative height of volatility smiles of Mark-Dollar call options (1985-1992) . . . . . . . . . 
3.1 Density estimates of the continuous time parameters $c_{1}=0.18, c_{2}=2$ and $c_{3}=1.2$ of the GARCH diffusion model (1.7)-(1.8) inferred by the parameter estimates of the discrete time GARCH-M model (3.1) at two-daily, daily and two-hourly sampling frequencies. Sample size 10 years. . . . . . . . . . . . . . . . . . . . . . .

3.2 Density estimates of the continuous time parameters $c_{1}=0.18, c_{2}=2$ and $c_{3}=1.2$ of the GARCH diffusion model (1.7)-(1.8) inferred by the parameter estimates of the discrete time GARCH-M model (3.1) at two-daily, daily and two-hourly sampling frequencies. Sample size

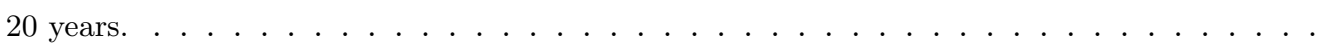

3.3 Density estimates of the continuous time parameters $c_{1}=0.18, c_{2}=2$ and $c_{3}=1.2$ of the GARCH diffusion model (1.7)-(1.8) inferred by the parameter estimates of the discrete time GARCH-M model (3.1) at two-daily, daily and two-hourly sampling frequencies. Sample size

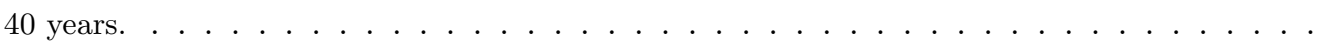

3.4 DM/US daily exchange rates from December 1988 to December 2003. $\sigma_{t}^{2}$ is defined in equations

3.5 DM/US daily exchange rates from January 1985 to October 1988. $\sigma_{t}^{2}$ is defined in equations

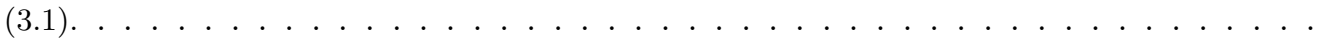




\section{List of Tables}

$2.1 P_{m c}$ Monte Carlo put prices computed by $N=10^{6}$ simulations; $\widetilde{P}^{g d(i)}$ GARCH diffusion put prices approximated by (2.10) truncated up to order $i$-th, for $i=2,3,4 ; e_{p}(i) \%=$ $100\left(\widetilde{P}^{g d(i)}-P_{m c}\right) / P_{m c} ; e_{m c}$ Monte Carlo standard error. Model parameters: $S_{0}=100$, $r=d=0 ; d V=(0.16-18 V) d t+1.8 V d W, V_{0}=0.16 / 18 \ldots \ldots \ldots \ldots$

$2.2 P_{m c}$ Monte Carlo put prices computed by $N=10^{6}$ simulations; $\widetilde{P}^{g d(i)}$ GARCH diffusion put prices approximated by (14) truncated up to order $i$-th, for $i=2,3,4 ; e_{p}(i) \%=$ $100\left(\widetilde{P}^{g d(i)}-P_{m c}\right) / P_{m c} ; e_{m c}$ Monte Carlo standard error. Model parameters: $S_{0}=100$, $r=d=0 ; d V=(0.53-29.23 V) d t+3.65 V d W, V_{0}=0.53 / 29.23 \ldots \ldots \ldots \ldots$

$2.3 P_{m c}$ Monte Carlo put prices computed by $N=10^{6}$ simulations; $\widetilde{P}^{g d(i)}$ GARCH diffusion put prices approximated by (14) truncated up to order $i$-th, for $i=2,3,4 ; e_{p}(i) \%=$ $100\left(\widetilde{P}^{g d(i)}-P_{m c}\right) / P_{m c} ; e_{m c}$ Monte Carlo standard error. Model parameters: $S_{0}=100$, $r=d=0 ; d V=(0.18-2 V) d t+0.8 V d W, V_{0}=0.18 / 2 \ldots \ldots \ldots \ldots$

$2.4 P_{m c}$ Monte Carlo put prices computed by $N=10^{6}$ simulations; $\widetilde{P}^{g d(i)}$ GARCH diffusion put prices approximated by (14) truncated up to order $i$-th, for $i=2,3,4 ; e_{p}(i) \%=$ $100\left(\widetilde{P}^{g d(i)}-P_{m c}\right) / P_{m c} ; e_{m c}$ Monte Carlo standard error. Model parameters: $S_{0}=100$, $r=d=0 ; d V=(0.18-2 V) d t+1.2 V d W, V_{0}=0.18 / 2 \ldots \ldots \ldots \ldots$ 
$2.5 P_{m c}$ Monte Carlo put prices computed by $N=10^{6}$ simulations; $\widetilde{P}^{g d(i)}$ GARCH diffusion put prices approximated by (14) truncated up to order $i$-th, for $i=2,3,4 ; e_{p}(i) \%=$ $100\left(\widetilde{P}^{g d(i)}-P_{m c}\right) / P_{m c} ; e_{m c}$ Monte Carlo standard error. Model parameters: $S_{0}=100$, $r=d=0 ; d V=(0.09-4 V) d t+1.2 V d W, V_{0}=0.09 / 4 \ldots \ldots \ldots . . . . . .$.

3.1 Bias RMSE First panel: sample size 10 years; second panel: sample size 20 years; third

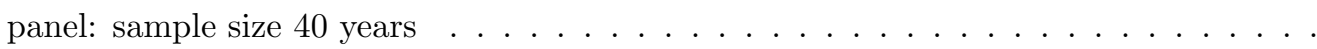

3.2 Summary statistics (mean, variance, kurtosis and inter quartile range) of DM/US exchange rates daily log-returns. . . . . . . . . . . . . . . . . .

3.3 GARCH(1,1)-M estimates for DM/US daily exchange rate from December 1988 to December 2003. $Z_{t}, \mu, \omega, \alpha$ and $\beta$ are defined in equations (3.1). ${ }^{a}$ Ljung-Box test follows $\chi^{2}(15)$ with the 0.05 critical value being $25 \ldots \ldots \ldots \ldots$

3.4 GARCH(1,1)-M estimates for DM/US daily exchange rates from January 1985 to October 1988. $Z_{t}, \mu, \omega, \alpha$ and $\beta$ are defined in equations (3.1). ${ }^{a}$ Ljung-Box test follows $\chi^{2}(15)$ with the 0.05 critical value being $25 \ldots \ldots \ldots \ldots . \ldots . \ldots$ 


\section{Introduction}

In this thesis, we study European option prices under a stochastic volatility model, where the asset price follows a geometric Brownian motion with instantaneous variance driven by a GARCH diffusion process.

Stochastic volatility models were introduced by Hull and White (1987), Scott (1987) and Wiggins (1987) to overcome some drawbacks of the Black and Scholes (1973) and Merton (1973) model. Since Mandelbrot (1963) and Fama (1965) it was kwon that speculative log-returns are uncorrelated, not independent with a leptokurtic distribution and conditional variance changing randomly over time. Moreover, empirical studies on implied volatility (cf., for instance, Lantane and Rendleman, 1976, Beckers, 1981, and Canina and Figlewski, 1993) show a significant discrepancy between market and Black and Scholes prices. Implied volatilities vary with strike price and time to maturity of the options contract whereas in the Black and Scholes model the volatility is assumed to be constant.

Stochastic volatility models allow to account for random behaviors of implied and historical variances and generate some log-returns features observed in empirical studies. Unfortunately, under stochastic volatilities closed or analytically tractable option pricing formulae are difficult to derive even for European options. The Hull and White (1987) and Heston (1993) models have an analytical approximation and a quasi analytical formula to price European options, respectively. For other stochastic volatility models numerical methods are used. 
In this thesis, we derive an analytical closed-form approximation for European option prices under the GARCH diffusion model, where the price is driven by a geometric process and the variance by an uncorrelated mean reverting geometric process. Our formula is related to the Hull and White (1987) option pricing series and involves the conditional moments of the integrated variance over the time to maturity. We show, using Monte Carlo simulations, that our approximation formula is accurate across several strike prices and times to maturity, it is easy to implement and allows to study the implied volatility and the volatility risk premia associated to the GARCH diffusion model.

The GARCH diffusion model is the 'mean reverting' extension of the Hull and White (1987) model where the variance follows a geometric Brownian motion and improves such a model under several aspects. The mean reverting drift gives stationary variance and log-returns processes (cf. GenonCatalot et al. (2000)) and allows to include the volatility risk premium in the variance process. On the contrary, for the Hull and White model the option pricing approximation is available only when the variance drift is equal to zero ${ }^{1}$. Furthermore, the mean reversion of the variance allows to approximate long maturity option prices, while the option pricing approximation for the Hull and White model holds only for short maturity options; cf. Hull and White (1987) and Gesser and Poncet (1997). This is a remarkable feature as in the last years long-term options are actively traded on option markets.

The Hull and White (1987) option pricing series holds only when the asset price and the variance processes are uncorrelated. This assumption implies symmetric volatility 'smiles', i.e. symmetric shapes of implied volatilities plotted versus strike prices; cf. Hull and White (1987) and Renault and Touzi (1996). As foreign currency option markets are characterized by symmetric volatility smiles (cf., for instance, Chesney and Scott 1989, Melino and Turbull 1990, Taylor and Xu 1994 and Bollerslev and Zhou 2002), the model considered in this thesis is appropriate to price currency options. Recent

\footnotetext{
${ }^{1}$ The volatility risk premium seems to be a significant component of the risk premia in many currency markets; cf. Guo (1998) and references therein.
} 
studies show that for some index option markets the non zero correlation between asset prices and variances can be neglected without increasing option pricing errors; cf. Chernov and Ghysels (2000) and Jones (2003) for empirical studies on Standard \& Poor's 500 and Standard \& Poor's 100, respectively.

The GARCH diffusion process driving the underlying asset price has several desirable properties. It is positive, mean reverting, with a stationary inverse Gamma distribution and satisfies the restriction that both historical and implied variances be positive. Hence, it fits the observation that empirical variances seem to be stationary and mean reverting; cf. Scott (1987), Taylor (1994), Jorion (1995) and Guo $(1996,1998)$. Moreover, the GARCH diffusion model allows for rich pattern behaviors of volatilities and asset prices. For instance, as observed in empirical studies, it produces large autocorrelation in the squared log-returns, arbitrary large kurtosis and finite unconditional moments of log-returns distributions up to some order. On the contrary, when the variance follows a square root process, as in the Heston (1993) model, the corresponding stationary Gamma distribution implies logreturn distributions with finite unconditional moments of any order and a 'not very large' kurtosis ${ }^{2}$ cf. Genon-Catalot, Jeantheau and Laredo (2000).

Furthermore, Nelson (1990) showed that a sequence of discrete time $\operatorname{GARCH}(1,1)$ in mean model (GARCH(1,1)-M; cf. Engle and Bollerslev 1986) converges in distribution to the GARCH diffusion model. Hence, the involved problem of making inference on continuous time parameters may be reduced to the inference on a GARCH(1,1)-M model; cf., for instance, Engle and Lee (1996) and Lewis (2000). This is an important advantage compared to other stochastic volatility models which do not share these properties. Using Monte Carlo simulations, we will investigate inference results based

\footnotetext{
${ }^{2}$ Empirical poor performances of the Heston model are reported by several authors, see for instance Andersen et al. (2002), Jones (2003) and reference therein. Jones (2003, p. 181) found that "conclude that the square root stochastic variance model of Heston [...] is incapable of generating realistic [equity index] returns behavior, and data are better represented by [other] stochastic variance model[s] in the CEV class", such as the GARCH diffusion model;
} 
on such estimation procedures.

The specific contributions of this thesis are the following. We derive analytically the first four exact conditional moments of the integrated variance implied by the GARCH diffusion process. This result has several important implications. First and foremost, these conditional moments allow us to obtain an analytical closed-form approximation for European option prices under the GARCH diffusion model. This approximation can be easily implemented in any standard software package. As we will show using Monte Carlo simulations, this approximation is very accurate across different strikes and maturities for a large set of reasonable parameters. Secondly, our analytical approximation allows to easily study volatility surfaces induced by GARCH diffusion models. Thirdly, the conditional moments of the integrated variance implied by the GARCH diffusion process generalize the conditional moments derived by Hull and White (1987) for log-normal variance processes. Finally, the conditional moments of the integrated variance can be used to estimate the continuous time parameters of the GARCH diffusion model using high frequency data ${ }^{3}$.

Outline. The thesis is organized as follows. Chapter 1 introduces stochastic volatility option pricing models and discusses in details the GARCH diffusion model and its properties. Chapter 2 presents the analytical approximation formula to price European options under the GARCH diffusion model. Using Monte Carlo simulations, we verify the accuracy of the approximation across different strike prices and times to maturity for different parameter choices. We investigate differences between option prices under the GARCH diffusion and the Black and Scholes model. Then, we qualitatively study implied volatility surfaces induced by the GARCH diffusion. Chapter 3 studies the accuracy of the

\footnotetext{
${ }^{3} \mathrm{By}$ matching the sample moments of the realized volatility with the conditional moments of the integrated variance one has a standard and easy to compute GMM-type estimator for the underlying model parameters; cf. Bollerslev and Zhou (2002).
} 
inference results on the GARCH diffusion model based on the Nelson's theory. Using such a procedure, we fit the GARCH diffusion model to daily log-returns of Deutsche Mark versus US dollar exchange rates. Chapter 4 gives some concluding remarks. 


\section{Chapter 1}

\section{Stochastic Volatility Models}

\subsection{Motivations of Stochastic Volatilities}

Black and Scholes (1973) and Merton (1973) derived a fundamental option pricing formula to price European options when the underlying asset price $S$ is driven by a geometric Brownian motion

$$
d S_{t}=\mu S_{t} d t+\sigma S_{t} d B_{t}, \quad S_{0}=S_{o}
$$

where $\mu \in \mathbf{R}, \sigma \in \mathbf{R}^{+}$and $B$ is a standard Brownian motion. Itô's lemma implies that the dynamic of $\ln S_{t}$ is given by

$$
d \ln S_{t}=\left(\mu-\sigma^{2} / 2\right) d t+\sigma d B_{t}
$$

Hence, the sequence of discrete time log-returns $Z_{i}$, sampled at time points $i \Delta, i=1, \ldots, \Delta>0$,

$$
Z_{i}:=\int_{(i-1) \Delta}^{i \Delta} d \ln S_{s}=\ln S_{i \Delta}-\ln S_{(i-1) \Delta}=\left(\mu-\sigma^{2} / 2\right) \Delta+\sigma^{2}\left(B_{i \Delta}-B_{(i-1) \Delta}\right)
$$

are independent and identically normally distributed with mean $\left(\mu-\sigma^{2} / 2\right) \Delta$ and variance $\sigma^{2} \Delta$. 
However, several empirical studies on asset prices show that speculative log-returns are uncorrelated, but not independent with leptokurtic distribution and conditional variance changing randomly over time. Moreover, the implied volatilities i.e. the volatility which gives the Black and Scholes price equals to the market price, are not constant across strike prices and time to maturities as assumed by the Black and Scholes model. Typically, for foreign currency options implied volatilities are too high for the at the money options and too low for out of the money and in the money options ("smile effect"). For stock and index options, Black and Scholes prices are too high for the in or the at the money options and too low for out of the money options ("smirk effect").

To overcome the drawbacks of the Black and Scholes model, several models have been proposed in the financial literature. Clearly, it is possible to generate the previous statistical properties for asset and option prices by allowing more involved drift and diffusion functions for $S$. However, such generalizations may occur at high costs, for instance intractable option pricing formulae or many parameters to estimate. A 'good model' for option pricing should retain as much as possible the simplicity of the Black and Scholes model and accounts for empirical the evidence on asset and option prices. Motivated by such empirical evidence, Hull and White (1987), Scott (1987) and Wiggins (1987) proposed stochastic volatility models as a parsimonious extension of the Black and Scholes model. These models retain the linearity of the drift and the diffusion components of the underlying asset introducing a second Itô process to drive the variance of $S$.

Stochastic volatility models, defined on some filtered probability space $\left(\Omega, \mathcal{F}, \mathcal{F}_{t}, \mathbb{P}\right)$, are given by the two dimensional stochastic differential equations,

$$
\begin{aligned}
& d S_{t}=\mu S_{t} d t+\sqrt{V_{t}} S_{t} d B_{t} \\
& d V_{t}=\beta\left(V_{t}, \theta\right) d t+\gamma\left(V_{t}, \theta\right) d W_{t}
\end{aligned}
$$

where $S_{t}$ is the underlying process, $V_{t}$ is the latent process for the variance of $d S_{t} / S_{t}$ depending on some 
parameter vector $\theta$, and $B_{t}, W_{t}$ are possibly correlated standard Brownian motions and $\left\{\mathcal{F}_{t}, t \geq 0\right\}$ is the filtration generated by $\left(B_{t}, W_{t}\right)$. Several authors, Wiggins (1987), Scott (1987), Chesney and Scott (1989), Hull and White (1988), Stein and Stein (1991) and Heston (1993), proposed stochastic volatility models where the variance of $d S_{t} / S_{t}$ follows a mean reverting process, while in the Hull and White (1987) model the variance follows a geometric Brownian motion. Stochastically changing volatilities over time allow to account for the random behaviors of implied and historical variances and generate some of the log-returns features observed in empirical studies. For instance, when the asset price and the variance processes are uncorrelated, discrete time log-returns $Z_{i}$ are uncorrelated, but not independent and with a leptokurtic distribution. Moreover, stochastic volatility accounts for smile and smirk effects observed on option markets. Under the assumption of no correlation between asset price and variance, Renault and Touzi (1996) (cf. also Hull and White (1987)) show that any stochastic volatility process induces symmetric volatility smiles usually observed in foreign option markets. When the asset price is correlated with the variance, stochastic volatility induces asymmetric smile, i.e. the smirk shape of implied volatility typically observed in stock and index markets; cf. Hull and White (1987), Heston (1990).

Stochastic volatility allows for more accurate and flexible option pricing models. Unfortunately, new difficulties arise both in the option pricing and in the parameter inference framework because of the latent variance process $V$. Option prices have to satisfy a bivariate partial differential equation in two state variables: the price and the variance of the underlying asset. Since the variance is not a traded asset, arbitrage arguments are not sufficient to uniquely determine option prices. Hence, the variance risk can not be hedged and the market price of variance risk explicitly enters into the partial differential equation. The associated risk premium is typically assumed to be zero or a constant proportion of variance. Analytical solutions of the bivariate partial differential equation are generally not available even for European options and numerical methods are used. Only the Hull and White (1987) model, 
where the variance follows a log-normal process

$$
d V_{t}=c_{2} V_{t} d t+c_{3} V_{t} d W_{t},
$$

and the Heston (1993) model, where the variance follows a squared root process

$$
d V_{t}=\left(c_{1}-c_{2} V_{t}\right) d t+c_{3} \sqrt{V} d W_{t} .
$$

have an analytical approximation formula and a quasi analytical formula to price European options, respectively. Hull and White show that, under a given risk adjusted measure, the price of a European call is the expected value of Black and Scholes price with squared volatility equals to the integrated variance over the time to maturity, $\bar{V}_{T}$. Based on this result, they derived an approximation option pricing formula in series form involving the conditional moments of $\bar{V}_{T}$.

Heston (1993) derives a quasi analytical option pricing formula to price European options in which correlation between asset price and variance processes is allowed. The derivation is based on Fourier inversion methods. Heston obtains an option pricing formula reminiscent of the Black and Scholes formulas involving risk neutral probabilities that are recovered from inverting the respective characteristic functions. This formula is quasi analytical as it involves integrals that have to be evaluated numerically.

For other stochastic volatility models, numerical methods are available but these procedures are computationally intensive and practically not feasible for instance are if large trading books have to be quickly and frequently evaluated many of these procedures. In this thesis we derive an analytical approximation formula to price European options when the asset price follows a GARCH diffusion model.

In the following section, we show the statistical properties GARCH diffusion model. 


\subsection{The GARCH Diffusion Model}

The GARCH diffusion model, first introduced by Wong (1964) and popularized ${ }^{1}$ by Nelson (1990), is defined as follows. Let $S=\left(S_{t}\right)_{t \geq 0}$ be the underlying currency price and $V=\left(V_{t}\right)_{t \geq 0}$ its latent instantaneous variance. $\left(S_{t}, V_{t}\right)_{t \geq 0}$ satisfies the two-dimensional GARCH diffusion model

$$
\begin{aligned}
& d S_{t}=\mu S_{t} d t+\sqrt{V_{t}} S_{t} d B_{t}, \\
& d V_{t}=\left(c_{1}-c_{2} V_{t}\right) d t+c_{3} V_{t} d W_{t},
\end{aligned}
$$

where $c_{1}, c_{2}$ and $c_{3}$ are positive constants, $\mu$ is the positive constant drift of $d S_{t} / S_{t}, B_{t}$ and $W_{t}$ are mutually independent one-dimensional Brownian motions on some filtered probability space $\left(\Omega, \mathcal{F}, \mathcal{F}_{t}, \mathbb{P}\right)$ with $\mathbb{P}$ is the objective measure. We set the initial time $t=0$ and $\left(S_{0}, V_{0}\right) \in \mathbb{R}^{+} \times \mathbb{R}^{+}$. The process $V$ is mean reverting, with $c_{1} / c_{2}$ the long-run mean value and $c_{2}$ the reversion rate (cf. also equation (1.10)). For $c_{2}$ small, the mean reversion is weak and $V_{t}$ tends to stay above (or below) the long-run mean value for long periods, i.e. it generates volatility cluster. The parameter $c_{3}$ determines the random behavior of the volatility: $c_{3}=0$ implies a deterministic volatility process, and $c_{3}>0$ a kurtosis of $\log$-return distributions larger than 3 . When $c_{1}=c_{2}=0$, the GARCH diffusion process reduces to the log-normal process without drift as in the Hull and White (1987) model.

Given $V_{0}>0, V_{t}$ is positive $\mathbb{P}$-almost surely, $\forall t \geq 0$, and the strong solution is

$$
V_{t}=V_{0} e^{-\left(c_{2}+\frac{1}{2} c_{3}^{2}\right) t+c_{3} W_{t}}+c_{1} \int_{0}^{t} e^{\left(c_{2}+\frac{1}{2} c_{3}^{2}\right)(s-t)+c_{3}\left(W_{t}-W_{s}\right)} d s
$$

The stationary distribution of $V$ is the Inverse Gamma distribution with parameters $r=1+2 c_{2} / c_{3}^{2}$ and $s=2 c_{1} / c_{3}^{2}$, i.e. $1 / V_{t} \leadsto \Gamma(r, s)$; cf. Nelson (1990).

\footnotetext{
${ }^{1}$ For more recent studies based on he GARCH diffusion model see, for instance, Lewis (2000), Melenberg and Werker (2001), Andersen, Bollerslev and Meddahi (2002) and references therein.
} 
When $2 c_{2}>c_{3}^{2}$, the process $V$ is strictly stationary, ergodic with conditional mean

$$
\mathbb{E}\left[V_{t} \mid V_{0}\right]=\frac{c_{1}}{c_{2}}+\left(V_{0}-\frac{c_{1}}{c_{2}}\right) e^{-c_{2} t}
$$

and variance

$$
\begin{aligned}
\operatorname{Var}\left[V_{t} \mid V_{0}\right]= & \frac{\left(c_{1} / c_{2}\right)^{2}}{2 c_{2} / c_{3}^{2}-1}+e^{-c_{2} t} \frac{2\left(c_{1} / c_{2}\right)\left(V_{0}-\left(c_{1} / c_{2}\right)\right)}{c_{2} / c_{3}^{2}-1}-e^{-2 c_{2} t}\left(V_{0}-\left(c_{1} / c_{2}\right)\right)^{2} \\
& +e^{\left(c_{3}^{2}-2 c_{2}\right) t}\left(V_{0}^{2}-\frac{2 V_{0}\left(c_{1} / c_{2}\right)}{1-c_{3}^{2} / c_{2}}+\frac{\left(c_{1} / c_{2}\right)^{2}}{\left(1-c_{3}^{2} / 2 c_{2}\right)\left(1-c_{3}^{2} / c_{2}\right)}\right)
\end{aligned}
$$

The unconditional expectation of (1.10) and (1.11) implies for the unconditional mean and variance of $\mathrm{V}$

$$
\mathbb{E}\left[V_{t}\right]=\frac{c_{1}}{c_{2}}, \quad \operatorname{Var}\left[V_{t}\right]=\frac{\left(c_{1} / c_{2}\right)^{2}}{2 c_{2} / c_{3}^{2}-1}
$$

For the Monte Carlo Simulation of Section 2.2, we use equations (1.12) to set some reasonable parameters for the variance process (1.8). Higher order unconditional moments of $V$ can be easily computed using the stationary Inverse Gamma distribution.

The GARCH diffusion model is an interesting and plausible specification of the variance process $\mathrm{V}$. It is positive, mean reverting, with a known stationary distribution. By Itô's lemma, the log-price dynamics implied by the GARCH diffusion model (1.7)-(1.8) is

$$
\begin{aligned}
d \ln S_{t} & =\left(\mu-\frac{V_{t}}{2}\right) d t+\sqrt{V_{t}} d B_{t} \\
d V_{t} & =\left(c_{1}-c_{2} V_{t}\right) d t+c_{3} V_{t} d W_{t} .
\end{aligned}
$$

The discrete time log-returns sampled at discrete time points $i \Delta, i=1, \ldots$, for a given time interval $\Delta>0$, are

$$
Z_{i}:=\int_{(i-1) \Delta}^{i \Delta} d \ln S_{s}=\ln S_{i \Delta}-\ln S_{(i-1) \Delta}
$$

The unconditional distribution of the process $Z_{i}$ is not known. But given the filtration $\sigma\left\{V_{s}, s \in[0, i]\right\}$, 
$Z_{i}$ is normally distributed, $Z_{i} \leadsto \mathcal{N}\left(M_{i}, \Sigma_{i}\right)$ where

$$
M_{i}:=\int_{(i-1) \Delta}^{i \Delta}\left(\alpha-\frac{V_{s}}{2}\right) d s \text { and } \Sigma_{i}:=\int_{(i-1) \Delta}^{i \Delta} V_{s} d s
$$

This model allows for rich volatilities and asset prices patterns and can explain some stylized facts observed in empirical studies. For convenience, we assume $M_{i}=0, \forall i$. Then,

$$
\begin{aligned}
& \mathbb{E}\left[Z_{i}\right]=0, \\
& \mathbb{E}\left[Z_{i}^{p} Z_{j}^{p}\right]=0, \quad \forall p \text { odd, } \\
& \operatorname{Cov}\left(Z_{i}, Z_{j}\right)=0, \\
& \mathbb{V a r} Z_{1}=\mathbb{E}\left[\Sigma_{1}\right]=\left(c_{1} / c_{2}\right) \Delta, \\
& \mathbb{V a r} Z_{1}^{2}=\mathbb{E}\left[Z_{1}^{4}\right]-\mathbb{E}\left[Z_{1}^{2}\right]^{2}=3 \mathbb{E}\left[\Sigma^{2}\right]-\left(c_{1} / c_{2}\right)^{2} \Delta^{2} \\
& \qquad=2\left(c_{1} / c_{2}\right)^{2} \Delta^{2}+\frac{6\left(c_{2} \Delta-1+e^{-c_{2} \Delta}\right)}{c_{2}^{2}} \frac{\left(c_{1} / c_{2}\right)^{2}}{\left(2 c_{2} / c_{3}^{2}\right)-1}, \\
& \mathbb{C o v}\left(Z_{1}^{2}, Z_{2}^{2}\right)=\frac{\left(1-e^{-c_{2} \Delta}\right)^{2}}{c_{2}^{2}} \frac{\left(c_{1} / c_{2}\right)^{2}}{\left(2 c_{2} / c_{3}^{2}\right)-1}
\end{aligned}
$$

and

$$
\operatorname{Corr}\left(Z_{1}^{2}, Z_{2}^{2}\right)=\frac{\left(1-e^{-c_{2} \Delta}\right)^{2} \frac{\left(c_{1} / c_{2}\right)^{2}}{\left(2 c_{2} / c_{3}^{2}\right)-1}}{2 c_{1}^{2} \Delta^{2}+6\left(c_{2} \Delta-1+e^{-c_{2} \Delta}\right) \frac{\left(c_{1} / c_{2}\right)^{2}}{\left(2 c_{2} / c_{3}^{2}\right)-1}}
$$

Equations (1.15) and (1.18) imply that $Z_{i}$ 's are uncorrelated but not independent. The excess-kurtosis $\mathbb{K}\left(Z_{1}\right)$ of the stationary distribution of the $Z_{i}$ process is given by

$$
\begin{aligned}
& \mathbb{K}\left(Z_{1}\right) \quad:=\frac{\mathbb{E} Z_{1}^{4}}{\left(\mathbb{E} Z_{1}^{2}\right)^{2}}-3 \\
& =\frac{6\left(c_{2} \Delta-1+e^{-c_{2} \Delta}\right)}{c_{2}^{2} \Delta^{2}} \frac{1}{\left(2 c_{2} / c_{3}^{2}\right)-1}>0 \\
& \operatorname{Corr}\left(Z_{1}^{2}, Z_{2}^{2}\right)<\frac{\frac{c_{3}^{2}}{2 c_{2}-c_{3}^{2}}}{2+3 \frac{c_{3}^{2}}{2 c_{2}-c_{3}^{2}}}=\frac{1}{\frac{2 c_{3}^{2}}{2 c_{2}-c_{3}^{2}}+3} .
\end{aligned}
$$


When $c_{3}^{2}$ tends to $2 c_{2}$, the kurtosis of log-return distributions diverges to infinity and the correlation between squared log-returns approaches $1 / 3$, i.e. the upper limit correlation of log-returns under mean reverting stochastic variance. Moreover, the Inverse Gamma stationary distribution of $V$ has finite moments up to order $r$ if and only if $r<1+2 c_{2} / c_{3}^{2}$, implying that log-returns distributions have finite unconditional moments up to order $2 r$. Some empirical studies show that (daily) log-returns distributions of several assets have not finite moments of all orders; cf., for instance, Dacorogna et al. (2001) and Cont (2001).

On the contrary, when the variance follows a square root process as in the Heston model (1993) the corresponding stationary Gamma distribution implies the log-return distributions of $Z_{i}$ with finite unconditional moments in any order. Moreover, in the Heston (1993) model the excess-kurtosis is at most 3 and the correlation between $Z_{1}^{2}$ and $Z_{2}^{2}$ at most $1 / 5$; cf. Genon-Catalot, Jeantheau and Laredo (2000). Therefore, the gamma distribution of $Z_{i}$ can be only moderately heavy-tailed compared to the empirical evidence.

An other important empirical observation is that periods of high volatilities and periods of more volatile volatilities tend to coincide; cf., for instance, Jones (2003). Hence, the volatility of volatility seems to be level dependent. Under the GARCH diffusion model, the volatility $\sigma_{t}:=\sqrt{V_{t}}$ follows the dynamics,

$$
d \sigma_{t}=\frac{1}{2}\left(\frac{c_{1}}{\sigma_{t}}-\left(c_{2}+\frac{c_{3}^{2}}{4}\right) \sigma_{t}\right) d t+\frac{c_{3}}{2} \sigma_{t} d W_{t}
$$

Hence, the volatility of volatility is level dependent. On the contrary, under the Heston (1993) model, the volatility $\sigma_{t}$ follows

$$
d \sigma_{t}=\frac{1}{2}\left(\frac{c_{1}-c_{3}^{2} / 4}{\sigma_{t}}-c_{2} \sigma_{t}\right) d t+\frac{c_{3}}{2} d W_{t}
$$

and the volatility of volatility is not level dependent. 


\section{Chapter 2}

\section{Option Pricing under the GARCH}

\section{Diffusion Model}

\subsection{The Option Pricing Formula}

In this section we derive an analytical approximation formula for European options when the underlying asset price satisfies equations (1.7)-(1.8). Since, the model (1.7)-(1.8) is appropriate to describe exchange rates dynamics, we specify our option pricing formula for currency options.

A currency option price $f(S, V, t)$ satisfies the following partial differential equation

$$
\frac{1}{2} V S^{2} \frac{\partial f^{2}}{\partial S^{2}}+\frac{1}{2} c_{3}^{2} V^{2} \frac{\partial f^{2}}{\partial V^{2}}+(r-d) S \frac{\partial f}{\partial S}+\left(\left(c_{1}-c_{2}\right) V-\lambda(S, V, t)\right) \frac{\partial f}{\partial V}-(r-d) f+\frac{\partial f}{\partial t}=0
$$

where $r$ and $d$ are the domestic and the foreign interest rates, respectively. The unspecified term $\lambda(S, V, t)$ represents the market price of risk associated to the variance $V$. As $V$ is not a traded asset, arbitrage arguments are not enough to determine uniquely the option price $f(S, V, t) . \lambda(S, V, t)$ has to be specified exogenously, for instance, based on consumption models; cf. Breeden (1979). As in other 
studies (cf., for instance, Chesney and Scott (1989), Heston (1993) and Jones (2003)), we specify the variance risk premium $\lambda(V, S, t)$ as a linear function ${ }^{1}$ of $V$,

$$
\lambda(S, V, t)=\bar{\lambda} V \quad \text { where } \quad \bar{\lambda}=\frac{c_{2}^{*}-c_{2}}{c_{3}} .
$$

The functional form of $\lambda$ in (2.1) implies that the risk-adjusted process is still a GARCH diffusion process,

$$
\begin{aligned}
& d S_{t}=\left(r_{d}-r_{f}\right) S_{t} d t+\sqrt{V_{t}} S_{t} d B_{t}^{*}, \\
& d V_{t}=\left(c_{1}-c_{2}^{*} V_{t}\right) d t+c_{3} V_{t} d W_{t}^{*},
\end{aligned}
$$

where $B^{*}$ and $W^{*}$ are mutually independent Brownian motions under the risk-adjusted measure $\mathbb{P}^{*}$. We show in Appendix A.3 that the Randon Nikodyn derivative $d \mathbb{P}^{*} /\left.d \mathbb{P}\right|_{\mathcal{F}_{t}}$ implied by the previous change of measure is well-defined.

Under the risk-adjusted dynamics in equations (2.2)-(2.3) the option pricing result in Hull and White (1987) holds: the price $C_{s v}$ for a European call with time to maturity $T$ and strike price $K$ is given by

$$
C_{s v}=\int_{0}^{\infty} C_{b s}\left(\bar{V}_{T}\right) f\left(\bar{V}_{T} \mid V_{0}\right) d \bar{V}_{T}
$$

where $C_{b s}$ is the Black and Scholes (1973) option price, $\bar{V}_{T}$ is the integrated variance over the time to maturity $T$,

$$
\bar{V}_{T}:=\frac{1}{T} \int_{0}^{T} V_{s} d s
$$

\footnotetext{
${ }^{1}$ Linearity of the variance risk premium can be supported under log-utility when exchange rate volatility and market risk have a common component of a particular form. The linear specification will not typically emerge under more general preferences (for instance, time separable power utility) and should be viewed for such preferences as an approximation to the true functional form. Cox Ingersoll and Ross (1985) use a similar approximation when modeling the risk premium in interest rates.
} 
and $f\left(\bar{V}_{T} \mid V_{0}\right)$ is the conditional density function of $\bar{V}_{T}$ given $V_{0}$. The density $f\left(\bar{V}_{T} \mid V_{0}\right)$ is not known and the option price $C_{s v}$ is not available in closed-form. The expectation in equation (2.4) can be computed by Monte Carlo simulation, but such a procedure is very time-consuming. Hull and White (1987) provide an analytical approximation for $C_{s v}$ in (2.4). They compute the Taylor expansion of $C_{b s}$ in equation (2.4) around the conditional mean of $\bar{V}_{T}$ obtaining a series option pricing formula that involves only the conditional moments of $\bar{V}_{T}$ and the sensitivities of the Black and Scholes price to the variance. Denoting by $M_{1}:=\mathbb{E}\left[\bar{V}_{T} \mid V_{0}\right]$ the conditional mean of $\bar{V}_{T}$ and by $M_{i c}:=\mathbb{E}\left[\left(\bar{V}_{T}-M_{1}\right)^{i} \mid V_{0}\right], \quad i \geq 2$, the $i$-th centered conditional moment of $\bar{V}_{T}$, the option pricing series is

$C_{s v}=C_{b s}\left(M_{1}\right)+\left.\frac{1}{2} M_{2 c} \frac{\partial^{2} C_{b s}}{\partial \bar{V}_{T}^{2}}\right|_{\bar{V}_{T}=M_{1}}+\left.\frac{1}{6} M_{3 c} \frac{\partial^{3} C_{b s}}{\partial \bar{V}_{T}^{3}}\right|_{\bar{V}_{T}=M_{1}}+\left.\frac{1}{24} M_{4 c} \frac{\partial^{4} C_{b s}}{\partial \bar{V}_{T}^{4}}\right|_{\bar{V}_{T}=M_{1}}+O\left(M_{5 c}\right)$

with the derivatives

$$
\begin{aligned}
\frac{\partial C_{b s}}{\partial \bar{V}_{T}}= & \frac{e^{-r_{f} T} S_{0} \sqrt{T} e^{-d_{1}^{2} / 2}}{\sqrt{8 \pi \bar{V}_{T}}}, \\
\frac{\partial^{2} C_{b s}}{\partial \bar{V}_{T}^{2}}= & \frac{\partial C_{b s}}{\partial \bar{V}_{T}}\left[\frac{1}{2} \frac{m^{2}}{\left(\bar{V}_{T} T\right)^{2}}-\frac{1}{2 \bar{V}_{T} T}-\frac{1}{8}\right] T, \\
\frac{\partial^{3} C_{b s}}{\partial \bar{V}_{T}^{3}}= & \frac{\partial C_{b s}}{\partial \bar{V}_{T}}\left[\frac{m^{4}}{4\left(\bar{V}_{T} T\right)^{4}}-\frac{m^{2}\left(12+\bar{V}_{T} T\right)}{8\left(\bar{V}_{T} T\right)^{3}}+\frac{48+8 \bar{V}_{T} T+\left(\bar{V}_{T} T\right)^{2}}{64\left(\bar{V}_{T} T\right)^{2}}\right] T^{2}, \\
\frac{\partial^{4} C_{b s}}{\partial \bar{V}_{T}^{4}}= & \frac{\partial C_{b s}}{\partial \bar{V}_{T}}\left[\frac{1}{8} \frac{m^{6}}{\left(\bar{V}_{T} T\right)^{6}}-\frac{3}{32} \frac{m^{4}\left(20+\bar{V}_{T} T\right)}{\left(\bar{V}_{T} T\right)^{5}}+\frac{3}{128} \frac{m^{2}\left(240+24 \bar{V}_{T} T+\left(\bar{V}_{T} T\right)^{2}\right)}{\left(\bar{V}_{T} T\right)^{4}}\right. \\
& \left.\quad-\frac{\left(960+144 \bar{V}_{T} T+12\left(\bar{V}_{T} T\right)^{2}+\left(\bar{V}_{T} T\right)^{3}\right)}{512\left(\bar{V}_{T} T\right)^{3}}\right] T^{3},
\end{aligned}
$$

and $m:=\log \left(S_{0} / K\right)+\left(r_{d}-r_{f}\right) T$. So far, the conditional moments of the integrated variance have been calculated analytically only for few specifications of the variance process,

1. for the mean reverting Ornstein-Uhlenbeck process ${ }^{2}$ Cox and Miller (1972, Sec. 5.8) derived the first two conditional moments of $\bar{V}_{T}$;

\footnotetext{
${ }^{2}$ Since the Ornstein-Uhlenbeck process is normally distributed, it can not ensure positive variance.
} 
2. for the geometric Brownian motion with drift Hull and White (1987) derived the first two conditional moments of $\bar{V}_{T}$ and the first three conditional moments of $\bar{V}_{T}$ for the variance process with zero drift;

3. for the squared root process Bollerslev and Zhou (2002) derived the first two conditional moments. Lewis (2000a) derived the first four conditional moments of the integrated variance for the general class of affine processes (including the squared root process).

Given the analytical conditional moments of $\bar{V}_{T}$ it is very easy to price European options by the series approximation in equation (2.6). Garcia et al. (2001) use this formula to price European options under the Heston model as an alternative to the Heston option pricing formula; cf. also Ball and Roma (1994). Indeed, implementing integral solutions for option prices, such as the Heston formula, can be very delicate due to divergence of the integrand in some regions of the parameter space.

We derive the second, the third and the fourth conditional moments of $\bar{V}_{T}$ when the variance $V$ is driven by the GARCH diffusion process (1.8). The first conditional moment was already known in the finance literature. For convenience, in the following proposition we state only the first two conditional moments. The third and the fourth conditional moments and the corresponding derivations will be made available to the interested reader upon request.

Higher order moments are essential to capture the 'smile' effect of implied volatilities; cf., for instance, Bodurtha and Courtadon (1987) for PHLX foreign currency options and Lewis (2000). We denote these conditional moments by $M_{1}^{g d}, M_{2 c}^{g d}, M_{3 c}^{g d}$ and $M_{4 c}^{g d}$. Here we state $M_{1}^{g d}, M_{2 c}^{g d}$. The calculations are given in Appendix A.1.

Proposition 2.1 Let $V=\left(V_{t}\right)_{t \geq 0}$ to satisfy the stochastic differential equation (1.8). Given $\left(V_{0}, c_{1}\right) \in$ $\mathbb{R}^{+} \times \mathbb{R}^{+}$and $c_{2}>c_{3}^{2}$, the first and the second conditional moments of the integrated variance $\bar{V}_{T}$ are

$$
M_{1}^{g d}:=\mathbb{E}\left[\bar{V}_{T} \mid V_{0}\right]=\frac{c_{1}}{c_{2}}+\left(V_{0}-\frac{c_{1}}{c_{2}}\right) \frac{1-e^{-c_{2} T}}{c_{2} T},
$$




$$
\begin{aligned}
M_{2 c}^{g d} & :=\mathbb{E}\left[\left(\bar{V}_{T}-M_{1}^{g d}\right)^{2} \mid V_{0}\right]=-\frac{e^{-2 T c_{2}}\left(c_{2} V_{0}-c_{1}\right)^{2}}{T^{2} c_{2}^{4}} \\
& +\frac{2 e^{\left(c_{3}^{2}-2 c_{2}\right) T}\left(2 c_{1}^{2}+2 c_{1}\left(c_{3}^{2}-2 c_{2}\right) V_{0}+\left(2 c_{2}^{2}-3 c_{2} c_{3}^{2}+c_{3}^{4}\right) V_{0}^{2}\right)}{T^{2}\left(c_{2}-c_{3}^{2}\right)^{2}\left(2 c_{2}-c_{3}^{2}\right)^{2}} \\
& -\frac{c_{3}^{2}\left(c_{1}^{2}\left(4 c_{2}\left(3-T c_{2}\right)+\left(2 T c_{2}-5\right) c_{3}^{2}\right)+2 c_{1} c_{2}\left(-2 c_{2}+c_{3}^{2}\right) V_{0}+c_{2}^{2}\left(c_{3}^{2}-2 c_{2}\right) V_{0}^{2}\right)}{T^{2} c_{2}^{4}\left(c_{3}^{2}-2 c_{2}\right)^{2}} \\
& +\frac{2 e^{-T c_{2}} c_{3}^{2}\left(2 c_{1}^{2}\left(T c_{2}^{2}-\left(1+T c_{2}\right) c_{3}^{2}\right)+2 c_{1} c_{2}^{2}\left(1-T c_{2}+T c_{3}^{2}\right) V_{0}+c_{2}^{2}\left(c_{3}^{2}-c_{2}\right) V_{0}^{2}\right)}{T^{2} c_{2}^{4}\left(c_{2}-c_{3}^{2}\right)^{2}} .
\end{aligned}
$$

These moments are obtained using properties of Brownian motion such as independence and stationarity of non-overlapping increments and the linearity of $d V_{t}$ in $V_{t}$. As already observed, when $c_{1}=0$ the GARCH diffusion process reduces to the log-normal process with drift and then $M_{1}^{g d}, M_{2 c}^{g d}$ reduce to the conditional mean and variance of $\bar{V}_{T}$ in Hull and White (1987), p. 287.

Given the first four conditional moments of $\bar{V}_{T}$, under the GARCH diffusion model the call price is

$$
\widetilde{C}^{g d}=C_{b s}\left(M_{1}^{g d}\right)+\left.\frac{M_{2 c}^{g d}}{2} \frac{\partial^{2} C_{b s}}{\partial \bar{V}_{T}^{2}}\right|_{\bar{V}_{T}=M_{1}^{g d}}+\left.\frac{M_{3 c}^{g d}}{6} \frac{\partial^{3} C_{b s}}{\partial \bar{V}_{T}^{3}}\right|_{\bar{V}_{T}=M_{1}^{g d}}+\left.\frac{M_{4 c}^{g d}}{24} \frac{\partial^{4} C_{b s}}{\partial \bar{V}_{T}^{4}}\right|_{\bar{V}_{T}=M_{1}^{g d}} .
$$

Although $M_{1}^{g d}, M_{2 c}^{g d}, M_{3 c}^{g d}$ and $M_{4 c}^{g d}$ are lengthy expressions, the closed-form approximation formula (2.10) can be easily implemented in any standard software package providing option prices by just plugging in model parameters without any computational efforts.

As we will show in the next section, our approximation formula (2.10) is very accurate for a large set of reasonable parameters. Intuitively, when the time to maturity $T$ is 'short', $\bar{V}_{T}$ is not too far from $M_{1}^{g d}=\mathbb{E}\left[\bar{V}_{T} \mid V_{0}\right]$, then we expect approximation (2.10) to converge quickly. When the time to maturity $T$ increases and the condition $2 c_{2}>3 c_{3}^{2}$ holds, for the stochastic strong law of large numbers ${ }^{3}$,

\footnotetext{
${ }^{3}$ The strong large numbers law is as follows. If $\pi$ is the limiting distribution of a stationary stochastic process $\mathrm{X}$ and $f$ is a real-valued function such that $\int|f(x)| \pi(x) d x<\infty$, then $\lim _{T \rightarrow+\infty} \frac{1}{T} \int_{0}^{T} f\left(X_{s}\right) d s=\int f(x) \pi(x) d x$ a.s.; cf., for instance, Bhattacharya and Waymire (1990), Theorem 12.2, p. 432. In our case, $f(X)=X$ and the stationary
} 
$\bar{V}_{T}$ tends to $c_{1} / c_{2}$, the mean value of the stationary distribution $V$, and $M_{2 c}, M_{3 c}$ and $M_{4 c}$ go to zero. Therefore, we expect the approximation formula (2.10) to work well also for 'long' maturities. On the contrary, in the Hull and White (1987) model, where the variance $V_{t}$ follows a log-normal process without drift, $M_{2 c}$ and $M_{3 c}$ tend to infinity when $T$ (see Appendix A.2) increases and the series (2.6) fails to give the right price; cf. Hull and White (1987) and Gesser and Poncet (1997). The effect of moving to a mean reverting process from a log-normal process is to avoid that the variance explodes or goes to zero when $T$ increases.

The conditions $2 c_{2}>3 c_{3}^{2}$ ensures that the stationary distribution of $V_{t}$ has finite moments (at least) up to order four. Hence, when $2 c_{2}$ approaches $3 c_{3}^{2}$, the formula $(2.10)$ becomes less accurate for long maturities, as for instance in Table (2.4), where the condition is violated. In this case, the variance process is 'too volatile', that is the volatility of volatility parameter $c_{3}$ is 'too large' and/or the mean reversion rate is too weak ( $c_{2}$ is 'too small'). However, this condition seems to be generally satisfied in options markets; cf. Section 2.2 .

Lewis (2000) derived a closed-form approximation for European option prices for a large class of stochastic volatility models including the GARCH diffusion model (2.2)-(2.3). Lewis's approximation formula is based on a second order Taylor expansion of some complex integrals around $c_{3}=0$; cf. Lewis (2000, pp. 77-84). Taking a Taylor expansion of the moments in our formula (2.10) around $c_{3}=0$ and truncating it at the second order only, would generate Lewis's formula. Therefore, for the GARCH diffusion model, our approximation is more accurate.

In the following section, we study by Monte Carlo simulations the accuracy of the approximation distribution of $V$ is the Inverse Gamma, $Y=1 / V \leadsto \Gamma(r, s)$ with density $\pi_{Y}(x)=s^{r} x^{r-1} e^{-s x} / \Gamma(r)$, where $r=$ $1+2 c_{2} / c_{3}^{2}$ and $s=2 c_{1} / c_{3}^{2}$. Then,

$\lim _{T \rightarrow+\infty} \bar{V}_{T}=\int v \pi_{V}(v) d v=\int \frac{1}{y} \pi_{Y}(y) d y=\int_{0}^{+\infty} \frac{1}{y} \frac{s^{r} y^{r-1} e^{-s y}}{\Gamma(r)} d y=\frac{c_{1}}{c_{2}} \int_{0}^{+\infty} \frac{s^{2 c_{2} / c_{3}^{2}} x^{\left(2 c_{2} / c_{3}^{2}\right)-1} e^{-s x}}{\Gamma\left(2 c_{2} / c_{3}^{2}\right)} d x=\frac{c_{1}}{c_{2}} a . s$. where we used $\Gamma\left(2 c_{2} / c_{3}^{2}+1\right)=2 c_{2} / c_{3}^{2} \Gamma\left(2 c_{2} / c_{3}^{2}\right)$. 
formula (2.10).

\subsection{Monte Carlo Simulations}

In order to verify the accuracy of approximation (2.10) we compute European option prices by Monte Carlo simulations. The advantage of using Monte Carlo estimates is that the standard error of estimates is known. Precisely, we compute put option prices ${ }^{4}$ according to equation (2.4) using the conditional Monte Carlo method; cf. Boyle, Broadie and Glasserman (1997).

Specifically, we divide the time interval $[0, T]$ into $s$ equal subintervals and we draw $s$ independent standard normal variables $\left(v_{i}\right)_{i=1, \ldots, s}$. We simulate the random variable $V_{t}$ in $(2.3)$ over the discrete time $i T / s$, for $i=1, \ldots, s$, using the Milstein scheme (cf. Kloeden and Platen (1999))

$$
V_{i}=c_{1} \Delta t+V_{i-1}\left(1-c_{2}^{*} \Delta t+c_{3} \sqrt{\Delta t} v_{i}\right)+\frac{1}{2} c_{3}^{2} V_{i-1}^{2}\left(\left(\sqrt{\Delta t} v_{i}\right)^{2}-\Delta t\right),
$$

where $\Delta t:=T / s$. Then, we compute the Black and Scholes put option price $P_{b s}^{(n)}$ with squared volatility $s^{-1} \sum_{i=1}^{s} V_{i}$. Finally, iterating this procedure $N$ times we obtain the Monte Carlo estimate for the put option price

$$
P_{m c}:=N^{-1} \sum_{n=1}^{N} P_{b s}^{(n)}
$$

with the corresponding Monte Carlo standard error

$$
e_{m c}:=\frac{\sqrt{N^{-1} \sum_{n=1}^{N}\left(P_{b s}^{(n)}-P_{m c}\right)^{2}}}{\sqrt{N}} .
$$

When $N$ goes to infinity, $P_{m c}$ converges in probability to the put option price implied by (2.4). Notice that we do not need to simulate the price process $S$.

\footnotetext{
${ }^{4}$ Monte Carlo standard errors are generally smaller for put option prices than for call option prices as in the first case payoffs are bounded. Using the put-call parity call option prices are readily computed.
} 
To simulate the variance process (2.3) we use parameter values inferred from empirical estimates of model (2.2)-(2.3). Typically, for currency and index daily log-returns the unconditional mean of $V$, $c_{1} / c_{2}^{*}$, ranges from 0.01 to 0.1 per year. The "half life ${ }^{5}$ varies from few days to about a half year; cf. Chesney and Scott (1989), Taylor and Xu (1994), Xu and Taylor (1994), Guo (1996, 1998) and Fouque, Papanicolaou and Sircar (2000). This implies that $c_{2}^{*}$ ranges from 1 to 40. Moreover, empirical estimates of discrete GARCH(1,1)-M model on currency and index daily log-returns imply values of $c_{3}$ ranging from about 1 to 4; cf., for instance, Hull and White (1987a, 1988) and Guo (1996,1998). For stock log-returns, estimates of $c_{3}$ are generally smaller. For the Monte Carlo simulations, we consider times to maturity for European put options ranging from 30 to 504 days. We wrote a Matlab code to long-run $N=10^{6}$ simulations. The computation time varies between about 14 hours for $T=30$ days to 15 hours for $T=504$ days on a PC Pentium IV $1 \mathrm{GHz}$, running Windows XP.

In Table 2.1 we simulate the risk-adjusted variance process (2.3) using parameter values that we infer (cf. Nelson (1990)) from the GARCH(1,1) estimates in Guo (1996) for the dollar/yen exchange rates $^{6}$, i.e. $c_{1}=0.16, c_{2}^{*}=18$ and $c_{3}=1.8$. The variance process is quickly mean reverting (the half life is about 10 trading days) and rather volatile, the two-standard deviation range for $V$ is from 0.003 to 0.014 ; see equations (1.12). Table 2.1 shows the Monte Carlo put price $P_{m c}$; the put price $\widetilde{P}^{g d(i)}$ given by the approximation formula (2.10) truncated up to order $i$-th, for $i=2,3$, 4 ; the corresponding pricing error $e_{p}(i) \%$ defined as $e_{p}(i) \%:=100\left(\widetilde{P}^{g d(i)}-P_{m c}\right) / P_{m c}$ and the Monte Carlo standard error $e_{m c}$. The pricing errors $e_{p}$ allow to evaluate the contributions of the different terms to option prices. The average pricing errors for $e_{p}(2) \%, e_{p}(3) \%$ and $e_{p}(4) \%$ are $-0.094,0.049$ and -0.067 ,

\footnotetext{
${ }^{5}$ The 'half life' is the time necessary after a shock to halve the deviation of $V_{t}$ from its long-run mean value, given that there are no more shocks. For this model the half life is equal to $\ln (2) / c_{2}^{*}$ years. We compute the half life using 252 trading days

${ }^{6}$ As in Guo (1996) we assume the volatility risk premium $\lambda(S, V, t)=0$.
} 
respectively. Although the variance process is rather volatile, the high mean reversion rate $c_{2}^{*}$ implies that the integrated variance process $\bar{V}_{T}$ tends to stay around $\mathbb{E}\left[\bar{V}_{T} \mid V_{0}\right]$ and that the approximation (2.10) works well. Indeed, almost all errors are practically negligible across all strikes and maturities, as they are within bid-ask spreads observed on currency option markets ${ }^{7}$.

In Table 2.2 we simulate the variance process (2.3) using the risk-neutral parameters reported by Melenberg and Werker (2001)for the Dutch EOE index. The variance risk premium was inferred using European call options on the Dutch index. The estimated correlation between price and volatility was negligible. The risk-neutral coefficients are $c_{1}=0.53, c_{2}^{*}=29.23$ and $c_{3}=3.65$. The long-run mean value of the variance is 0.018 and the two-standard deviation range for $V$ is $0-0.038$. Table 2.2 is organized as Table 2.1. The average pricing errors for $e_{p}(2) \%, e_{p}(3) \%$ and $e_{p}(4) \%$ are $0.129,0.010$ and -0.174 , respectively. In this case pricing errors $e_{p}(3) \%$ are almost always lower than $1 \%$ (except one case). Unfortunately, for some parameter choice, the fourth term in (2.10) can be highly unstable across times to maturity and strike prices, due to the high variability of the fourth derivative of the Black and Scholes price. Hence, even though $M_{3}^{g d} \gg M_{4}^{g d}, e_{p}(3)$ tend to be smaller than $e_{p}(4)$ pricing errors.

In Table 2.3 and Table 2.4 we use parameter values that give a reasonable variance process as discussed in Hull and White (1988). In Tables 2.3 we set $c_{1}=0.18, c_{2}^{*}=2$ and $c_{3}=0.8$. The parameter value $c_{2}^{*}$ is quite small and implies a 'slow' mean reverting variance process (2.3) with half life of about 87 trading days. The unconditional mean and standard deviation of $V$ are 0.090 and 0.039 , respectively, and the two-standard deviation range for $V$ is $0.011-0.169$. As the volatility of $V_{t}$ is not too large, the process $\bar{V}_{T}$ tends to stay around $\mathbb{E}\left[\bar{V}_{T} \mid V_{0}\right]$ and hence the series approximation (2.10) is very accurate. The average pricing errors for $e_{p}(2) \%, e_{p}(3) \%$ and $e_{p}(4) \%$ are $-0.035,0.044$ and

\footnotetext{
${ }^{7}$ Typically, bid-ask spreads on currency options are larger than $2 \%$ of option prices for out of the money options and about $1 \%$ for more liquid at the money options.
} 
-0.018 , respectively.

In Table 2.4 we set $c_{1}$ and $c_{2}^{*}$ as in Table 2.3 and $c_{3}=1.2$. This implies that the standard deviation of $\mathrm{V}$ is 0.068 and the two-standard deviation range for $V$ is $0-0.225$. The average pricing errors for $e_{p}(2) \%, e_{p}(3) \%$ and $e_{p}(4) \%$ are $-0.246,0.401$ and -1.412 , respectively. The errors $e_{p}(3)$ are still very small, but slightly larger than in Table 2.3 as the variance process is more volatile than in the previous case. The pricing errors $e_{p}(4) \%$ are very large, especially for long maturities, because the fourth unconditional moment of $V_{t}$ is not finite as the condition $2 c_{2}>3 c_{3}^{2}$ does not hold.

Finally, in Table 2.5 we set $c_{1}=0.09, c_{2}^{*}=4$ and $c_{3}=1.2$ as in Lewis (2000). The unconditional mean of $V$ is 0.022 , the 'half life' is about 44 trading days and the two-standard deviation range for $V$ is $0.001-0.043$. Also in this case pricing errors are generally quite small and the averages for $e_{p}(2) \%$, $e_{p}(3) \%$ and $e_{p}(4) \%$ are $0.024,0.020$ and 0.053 , respectively.

The simulation results reported in Tables 2.1-2.5 show that, for some short maturities and deep out of the money options, $\widetilde{P}^{g d(2)}$ prices are not enough accurate, as pricing errors $e_{p}(2)$ are larger than bid-ask tolerance. Using the option price approximation $\widetilde{P}^{g d(3)}$ reduces the previous largest pricing errors and generally gives errors $e_{p}(3)$ within bid-ask spreads. In some cases (cf. Tables 2.1-2.2), due to the instability of the fourth term in approximation formula (2.10), the option price approximation $\widetilde{P}^{g d(4)}$ gives large pricing errors. Therefore, it seems to be convenient to truncate after three terms the option pricing formula (2.10) for a simpler and, in some cases, more precise approximation.

Although $e_{p}$ pricing errors in Tables $2.1-2.5$ are generally very small, such errors seems to be display some patterns especially for long maturities. A possible explanation is as follows. The Black and Scholes put price, which is the first term in the approximation formula given by (2.10), tend to be larger than the Monte Carlo put prices. The second term in the put option price $\widetilde{P}^{g d(2)}$ is almost always negative reducing the price bias of the Black and Scholes formula and partially explaining the negative pricing errors $e_{p}(2)$ in Tables $2.3-2.5$. The third term in $\widetilde{P}^{g d(3)}$ is almost always positive 
explaining the positive pricing errors $e_{p}(3)$ observed in the tables. The contribution of the fourth term in $\widetilde{P}^{g d(4)}$ has not a clear sign.

We simulate the variance process (2.3) also for other reasonable parameter choices (not reported here) and we find similar results. The approximation formula $\widetilde{P}^{g d(3)}$ induces pricing errors smaller than $1 \%$ for at the money options and smaller than $2 \%$ for out of the money options. Then, this approximation formula gives accurate prices within the tolerance expected because of market frictions.

\subsection{Effects of Stochastic Volatility on Option Prices}

In this section, we investigate the effects of the stochastic volatility driven by the GARCH diffusion process (2.2)-(2.3) on European option prices. We study the so-called 'price bias', that is the difference between option prices under stochastic volatility and option prices under deterministic volatility for different parameter choices of $c_{1}, c_{2}^{*}$ and $c_{3}$, across several strikes and maturities.

In the deterministic volatility setting $c_{3}=0$, Merton (1973) showed that option prices are simply given by the Black and Scholes formula with the modified volatility

$$
\sigma_{d e t}^{2}:=\frac{1}{T} \int_{0}^{T}\left(c_{1}-c_{2}^{*} V_{s}\right) d s=\frac{c_{1}}{c_{2}^{*}}+\left(V_{0}-\frac{c_{1}}{c_{2}^{*}}\right) \frac{1-e^{-c_{2}^{*} T}}{c_{2}^{*} T}
$$

which is indeed the first conditional moment of $\bar{V}_{T}, M_{1}^{g d}$. In our experiments, we set $c_{1}=0.09$, $c_{2}^{*}=4$ and $c_{3}=1.2$ as in Lewis (2000); cf. also Tables 2.5-??. Similar results (not reported here) are obtained for other parameter values.

Figure 2.1 shows call option price biases for different values of the parameter $c_{3}$, which controls the volatility of volatility. As expected, the main effect of moving from deterministic to stochastic volatilities is to decrease near-the-money option prices and to increase out-of-the-money and in-themoney option prices; cf., for instance, Hull and White (1987) and Heston (1993). When $c_{3}$ increases the volatility and the kurtosis of the log-return distributions increases raising in the money and out of 
the money option prices and lowering at the money option prices. This effect shrinks for deep in the money and deep out of the money options, as option prices tend to be insensitive to the volatility.

Figure 2.2 shows price biases for different values of $c_{2}^{*}$. As $c_{3} \neq 0$, the shapes of price biases are similar to Figure 2.1. When $c_{2}^{*}$ increases, the variance process $V$ becomes less volatile for two reasons: the long-run mean value of $V$ reduces and the reversion rate increases. This effect tend to reduce the price biases, across all strikes and maturities.

Figure 2.3 shows the effect of $c_{1}$ on price biases. When $c_{1}$ increases the long-run mean value of $V$ increases and price biases tend to become more negative for in the money and out of the money option prices. For at the money option prices the evidence is mixed.

Finally, Figure 2.4 shows price biases for different values of the initial variance $V_{0}$. The larger $V_{0}$, the more volatile is the variance process. The effect on the price biases of increasing $V_{0}$ is quite similar to the effect of increasing $c_{3}$.

When the time to maturity increases, the negative price bias for at the money options becomes more pronounced and holds for a wider range of strike prices. Only for 'very long' maturities (about five years), the price bias is roughly zero for all strike prices. Then, the GARCH diffusion and the deterministic volatility model tend to coincide, as the integrated variance $\bar{V}_{T}$ and $M_{1}^{g d}$ are very close to the long-run mean value $c_{1} / c_{2}^{*}$.

\subsection{Implied Volatility Surfaces}

In this section we study the implied volatility induced by the GARCH diffusion model (2.2)-(2.3), i.e. the volatility $\sigma_{i m p}^{2}$ which gives the Black and Scholes option price equals to the GARCH diffusion

option price, $C_{b s}\left(\sigma_{i m p}^{2}\right)=\widetilde{C}^{g d}$. Typically, to solve such implicit equation the Newton-Raphson method 
is used $^{8}$.

Renault and Touzi (1996) show that, for any stochastic volatility process, the assumption of no correlation between price and variance induces symmetric 'volatility smiles', i.e. symmetric shape of implied volatilities with respect to $m=\ln \left(S_{0} / K\right)+(r-d) T$ as a function of the strike prices; cf. also Hull and White (1987). The functional dependence of implied volatilities on time to maturity, i.e. the 'term structure pattern', depends on the specific variance process. In the following we study qualitatively the volatility smile and the term structure pattern induced by the GARCH diffusion model. As in Table 2.5 we set $c_{1}=0.09, c_{2}^{*}=4$ and $c_{3}=1.2$ and we compute the GARCH diffusion option prices (2.10) and the implied volatilities for different strikes and maturities. Figure 2.5 shows volatility smiles for time to maturities equal to 30,60, 90 and 120 days. Figure 2.6 shows the volatility surface for time to maturity between 30 and 120 days and strike prices between 90 and 110 . As the implied volatility is symmetric with respect to $m$, volatility smiles are quite symmetric with respect to the forward price. Moreover, the convexity of the volatility surface increases when the time to maturity decreases. These features of implied volatility surface were observed for all parameter choices (positive parameters). When the time to maturity increases the volatility surface flattens because the random variable $\bar{V}_{T}$ converges to the the long-run mean value $c_{1} / c_{2}^{*}$ by the stochastic strong law of large number and $\sigma_{i m p}^{2} \rightarrow c_{1} / c_{2}^{*}$ for all strike prices. These results are in qualitative agreement with the empirical evidence on volatility surfaces observed in currency option markets, where volatility smiles are quite symmetric with respect to the forward price, very pronounced at short maturities and almost flat at long maturities; cf., for instance, Chesney and Scott (1989), Melino and Turbull (1990), Taylor

\footnotetext{
${ }^{8}$ See for instance the Matlab function blsimpvdiv or the Mathematica function BlackScholesCallImpVol. An approximation for the implied volatility is $\sigma_{i m p}^{2} \approx M_{1}^{g d}+\left(\widetilde{C}^{g d}-C_{b s}\left(M_{1}^{g d}\right)\right) / \partial C_{b s} / \partial \bar{V}_{T} \bar{V}_{T}=M_{1}^{g d}$, that is a one-step Newton-Raphson algorithm starting at $M_{1}^{g d}$. As $\sigma_{i m p}^{2} \rightarrow M_{1}^{g d}$ for $T \rightarrow \infty, M_{1}^{g d}$ is a reasonable starting point for the algorithm and indeed, one iteration provides rather accurate results.
} 
and Xu (1994), and Bollerslev and Zhou (2002). For instance, Taylor and Xu (1994) estimated the relative height of volatility smiles for Deutsche mark versus US dollar option prices from 1985-1994. The results are reported in Figure 2.7. Clearly, the volatility smiles are quite symmetric and more convex for short maturities ${ }^{9}$.

${ }^{9}$ Taylor and $\mathrm{Xu}$ found similar volatility smiles for other foreign exchange call options on Pound-Dollar, Yen-Dollar, Swiss-Dollar exchange rates. 


\begin{tabular}{|c|c|c|c|c|c|c|c|c|c|}
\hline$T$ & $K$ & $P_{m c}$ & $\widetilde{P}^{g d(2)}$ & $\widetilde{P}^{g d(3)}$ & $\widetilde{P}^{g d(4)}$ & $e_{p}(2) \%$ & $e_{p}(3) \%$ & $e_{p}(4) \%$ & $e_{m c} \times 10^{4}$ \\
\hline \multirow[t]{5}{*}{30} & 90 & 0.0008 & 0.0008 & 0.0008 & 0.0008 & -4.5641 & 1.3768 & 0.6479 & 0.0129 \\
\hline & 95 & 0.0800 & 0.0803 & 0.0800 & 0.0801 & 0.2754 & -0.1116 & 0.0948 & 0.3610 \\
\hline & 100 & 1.2921 & 1.2918 & 1.2924 & 1.2919 & -0.0177 & 0.0269 & -0.0084 & 1.1997 \\
\hline & 105 & 5.0999 & 5.1002 & 5.0998 & 5.1000 & 0.0046 & -0.0022 & -0.0022 & 0.4231 \\
\hline & 110 & 10.0023 & 10.0023 & 10.0024 & 10.0023 & -0.0003 & 0.0003 & -0.0004 & 0.0294 \\
\hline \multirow[t]{5}{*}{60} & 90 & 0.0184 & 0.0184 & 0.0184 & 0.0184 & 0.3769 & 0.2996 & 0.1640 & 0.1330 \\
\hline & 95 & 0.2993 & 0.2995 & 0.2992 & 0.2993 & 0.0859 & -0.0230 & 0.0033 & 0.8289 \\
\hline & 100 & 1.8284 & 1.8283 & 1.8290 & 1.8288 & 0.0076 & 0.0275 & 0.0212 & 1.5411 \\
\hline & 105 & 5.3497 & 5.3499 & 5.3496 & 5.3497 & 0.0047 & -0.0011 & 0.0005 & 0.9216 \\
\hline & 110 & 10.0363 & 10.0365 & 10.0364 & 10.0363 & 0.0015 & 0.0004 & 0.0002 & 0.2225 \\
\hline \multirow[t]{5}{*}{90} & 90 & 0.0664 & 0.0665 & 0.0664 & 0.0664 & 0.2415 & -0.0006 & -0.0512 & 0.3067 \\
\hline & 95 & 0.5401 & 0.5403 & 0.5401 & 0.5392 & 0.0263 & 0.0004 & -0.1771 & 1.0964 \\
\hline & 100 & 2.2411 & 2.2409 & 2.2414 & 2.2432 & -0.0062 & 0.0164 & 0.0957 & 1.6780 \\
\hline & 105 & 5.6155 & 5.6156 & 5.6155 & 5.6146 & 0.0023 & 0.0004 & -0.0156 & 1.1971 \\
\hline & 110 & 10.1131 & 10.1133 & 10.1131 & 10.1127 & 0.0022 & -0.0003 & -0.0046 & 0.4537 \\
\hline \multirow[t]{5}{*}{120} & 90 & 0.1393 & 0.1395 & 0.1393 & 0.1380 & 0.1276 & -0.0119 & -0.9736 & 0.4727 \\
\hline & 95 & 0.7760 & 0.7761 & 0.7760 & 0.7745 & 0.0137 & 0.0096 & -0.1902 & 1.2603 \\
\hline & 100 & 2.5890 & 2.5889 & 2.5893 & 2.5937 & -0.0026 & 0.0124 & 0.1821 & 1.7489 \\
\hline & 105 & 5.8721 & 5.8722 & 5.8722 & 5.8709 & 0.0017 & 0.0016 & -0.0205 & 1.3634 \\
\hline & 110 & 10.2187 & 10.2189 & 10.2187 & 10.2163 & 0.0021 & -0.0003 & -0.0233 & 0.6541 \\
\hline \multirow[t]{5}{*}{180} & 90 & 0.3294 & 0.3296 & 0.3294 & 0.3239 & 0.0601 & 0.0165 & -1.6659 & 0.7371 \\
\hline & 95 & 1.2161 & 1.2163 & 1.2163 & 1.2158 & 0.0138 & 0.0174 & -0.0274 & 1.4470 \\
\hline & 100 & 3.1725 & 3.1726 & 3.1729 & 3.1819 & 0.0035 & 0.0112 & 0.2969 & 1.8187 \\
\hline & 105 & 6.3462 & 6.3464 & 6.3465 & 6.3466 & 0.0027 & 0.0037 & 0.0052 & 1.5509 \\
\hline & 110 & 10.4733 & 10.4735 & 10.4734 & 10.4667 & 0.0021 & 0.0007 & -0.0632 & 0.9500 \\
\hline \multirow[t]{5}{*}{252} & 90 & 0.5928 & 0.5930 & 0.5929 & 0.5845 & 0.0243 & 0.0109 & -1.3950 & 0.9571 \\
\hline & 95 & 1.6919 & 1.6921 & 1.6921 & 1.6942 & 0.0071 & 0.0106 & 0.1333 & 1.5684 \\
\hline & 100 & 3.7550 & 3.7551 & 3.7553 & 3.7678 & 0.0026 & 0.0068 & 0.3387 & 1.8616 \\
\hline & 105 & 6.8550 & 6.8551 & 6.8552 & 6.8582 & 0.0018 & 0.0028 & 0.0464 & 1.6719 \\
\hline & 110 & 10.8062 & 10.8063 & 10.8063 & 10.7977 & 0.0015 & 0.0008 & -0.0784 & 1.1822 \\
\hline \multirow[t]{5}{*}{504} & 90 & 1.5438 & 1.5440 & 1.5440 & 1.5387 & 0.0111 & 0.0108 & -0.3345 & 1.3327 \\
\hline & 95 & 3.0577 & 3.0579 & 3.0579 & 3.0660 & 0.0065 & 0.0078 & 0.2713 & 1.7337 \\
\hline & 100 & 5.3116 & 5.3118 & 5.3119 & 5.3273 & 0.0040 & 0.0051 & 0.2951 & 1.9142 \\
\hline & 105 & 8.3052 & 8.3054 & 8.3054 & 8.3145 & 0.0025 & 0.0031 & 0.1126 & 1.8354 \\
\hline & 110 & 11.9443 & 11.9445 & 11.9445 & 11.9410 & 0.0016 & 0.0017 & -0.0276 & 1.5585 \\
\hline
\end{tabular}

Table 2.1: $P_{m c}$ Monte Carlo put prices computed by $N=10^{6}$ simulations; $\widetilde{P}^{g d(i)}$ GARCH diffusion put prices approximated by (2.10) truncated up to order $i$-th, for $i=2,3,4 ; e_{p}(i) \%=100\left(\widetilde{P}^{g d(i)}-\right.$ $\left.P_{m c}\right) / P_{m c} ; e_{m c}$ Monte Carlo standard error. Model parameters: $S_{0}=100, r=d=0 ; d V=$ $(0.16-18 \mathrm{~V}) d t+1.8 \mathrm{VdW}, V_{0}=0.16 / 18$. 


\begin{tabular}{|c|c|c|c|c|c|c|c|c|c|}
\hline$T$ & $K$ & $P_{m c}$ & $\widetilde{P}^{g d(2)}$ & $\widetilde{P}^{g d(3)}$ & $\widetilde{P}^{g d(4)}$ & $e_{p}(2) \%$ & $e_{p}(3) \%$ & $e_{p}(4) \%$ & $e_{m c} \times 10^{4}$ \\
\hline \multirow[t]{5}{*}{30} & 90 & 0.0236 & 0.0242 & 0.0240 & 0.0219 & 2.5477 & 1.5083 & -7.1357 & 0.3503 \\
\hline & 95 & 0.3093 & 0.3107 & 0.3071 & 0.3137 & 0.4467 & -0.7134 & 1.3975 & 1.5033 \\
\hline & 100 & 1.8344 & 1.8317 & 1.8389 & 1.8294 & -0.1445 & 0.2473 & -0.2728 & 2.6253 \\
\hline & 105 & 5.3602 & 5.3614 & 5.3580 & 5.3647 & 0.0235 & -0.0398 & 0.0843 & 1.6594 \\
\hline & 110 & 10.0436 & 10.0449 & 10.0435 & 10.0421 & 0.0123 & -0.0016 & -0.0151 & 0.5242 \\
\hline \multirow[t]{5}{*}{60} & 90 & 0.1508 & 0.1523 & 0.1495 & 0.1508 & 1.0367 & -0.8793 & 0.0362 & 0.9954 \\
\hline & 95 & 0.7899 & 0.7902 & 0.7898 & 0.7912 & 0.0313 & -0.0177 & 0.1651 & 2.3314 \\
\hline & 100 & 2.6014 & 2.5995 & 2.6052 & 2.6010 & -0.0721 & 0.1464 & -0.0173 & 3.1471 \\
\hline & 105 & 5.8867 & 5.8868 & 5.8868 & 5.8880 & 0.0018 & 0.0017 & 0.0221 & 2.5150 \\
\hline & 110 & 10.2325 & 10.2343 & 10.2307 & 10.2331 & 0.0177 & -0.0176 & 0.0054 & 1.3279 \\
\hline \multirow[t]{5}{*}{90} & 90 & 0.3449 & 0.3461 & 0.3438 & 0.3414 & 0.3371 & -0.3171 & -1.0108 & 1.4788 \\
\hline & 95 & 1.2348 & 1.2347 & 1.2355 & 1.2353 & -0.0043 & 0.0563 & 0.0419 & 2.7146 \\
\hline & 100 & 3.1915 & 3.1904 & 3.1942 & 3.1981 & -0.0354 & 0.0859 & 0.2068 & 3.3572 \\
\hline & 105 & 6.3659 & 6.3657 & 6.3668 & 6.3669 & -0.0022 & 0.0139 & 0.0154 & 2.9048 \\
\hline & 110 & 10.4914 & 10.4926 & 10.4903 & 10.4874 & 0.0113 & -0.0108 & -0.0380 & 1.8716 \\
\hline \multirow[t]{5}{*}{120} & 90 & 0.5668 & 0.5675 & 0.5661 & 0.5596 & 0.1359 & -0.1128 & -1.2677 & 1.8267 \\
\hline & 95 & 1.6394 & 1.6393 & 1.6403 & 1.6417 & -0.0071 & 0.0526 & 0.1408 & 2.9359 \\
\hline & 100 & 3.6891 & 3.6883 & 3.6911 & 3.7009 & -0.0197 & 0.0555 & 0.3199 & 3.4717 \\
\hline & 105 & 6.7987 & 6.7985 & 6.7997 & 6.8018 & -0.0026 & 0.0147 & 0.0465 & 3.1284 \\
\hline & 110 & 10.7723 & 10.7730 & 10.7717 & 10.7649 & 0.0068 & -0.0049 & -0.0682 & 2.2435 \\
\hline \multirow[t]{5}{*}{180} & 90 & 1.0310 & 1.0316 & 1.0311 & 1.0225 & 0.0546 & 0.0083 & -0.8305 & 2.2774 \\
\hline & 95 & 2.3550 & 2.3552 & 2.3561 & 2.3621 & 0.0082 & 0.0457 & 0.3029 & 3.1795 \\
\hline & 100 & 4.5229 & 4.5229 & 4.5245 & 4.5405 & -0.0004 & 0.0361 & 0.3886 & 3.5916 \\
\hline & 105 & 7.5601 & 7.5603 & 7.5613 & 7.5685 & 0.0024 & 0.0156 & 0.1106 & 3.3733 \\
\hline & 110 & 11.3372 & 11.3378 & 11.3375 & 11.3300 & 0.0049 & 0.0024 & -0.0635 & 2.7073 \\
\hline \multirow[t]{5}{*}{252} & 90 & 1.5777 & 1.5781 & 1.5780 & 1.5718 & 0.0262 & 0.0217 & -0.3725 & 2.6024 \\
\hline & 95 & 3.0980 & 3.0983 & 3.0990 & 3.1089 & 0.0095 & 0.0314 & 0.3520 & 3.3406 \\
\hline & 100 & 5.3549 & 5.3551 & 5.3561 & 5.3748 & 0.0044 & 0.0239 & 0.3731 & 3.6742 \\
\hline & 105 & 8.3478 & 8.3481 & 8.3488 & 8.3600 & 0.0036 & 0.0126 & 0.1466 & 3.5354 \\
\hline & 110 & 11.9831 & 11.9835 & 11.9836 & 11.9796 & 0.0036 & 0.0042 & -0.0288 & 3.0333 \\
\hline \multirow[t]{5}{*}{504} & 90 & 3.2715 & 3.2723 & 3.2724 & 3.2751 & 0.0230 & 0.0271 & 0.1099 & 3.0858 \\
\hline & 95 & 5.1528 & 5.1536 & 5.1540 & 5.1681 & 0.0167 & 0.0228 & 0.2963 & 3.5523 \\
\hline & 100 & 7.5754 & 7.5763 & 7.5767 & 7.5957 & 0.0120 & 0.0172 & 0.2683 & 3.7759 \\
\hline & 105 & 10.5187 & 10.5196 & 10.5199 & 10.5352 & 0.0086 & 0.0118 & 0.1567 & 3.7472 \\
\hline & 110 & 13.9310 & 13.9318 & 13.9320 & 13.9375 & 0.0061 & 0.0075 & 0.0468 & 3.5050 \\
\hline
\end{tabular}

Table 2.2: $P_{m c}$ Monte Carlo put prices computed by $N=10^{6}$ simulations; $\widetilde{P}^{g d(i)}$ GARCH diffusion put prices approximated by (14) truncated up to order $i$-th, for $i=2,3,4 ; e_{p}(i) \%=100\left(\widetilde{P}^{g d(i)}-P_{m c}\right) / P_{m c}$; $e_{m c}$ Monte Carlo standard error. Model parameters: $S_{0}=100, r=d=0 ; d V=(0.53-29.23 V) d t+$ $3.65 V d W, V_{0}=0.53 / 29.23$. 


\begin{tabular}{|c|c|c|c|c|c|c|c|c|c|}
\hline$T$ & $K$ & $P_{m c}$ & $\widetilde{P}^{g d(2)}$ & $\widetilde{P}^{g d(3)}$ & $\widetilde{P}^{g d(4)}$ & $e_{p}(2) \%$ & $e_{p}(3) \%$ & $e_{p}(4) \%$ & $e_{m c} \times 10^{4}$ \\
\hline \multirow[t]{5}{*}{30} & 90 & 0.7901 & 0.7902 & 0.7900 & 0.7876 & 0.0141 & -0.0099 & -0.3165 & 1.6735 \\
\hline & 95 & 2.0011 & 2.0008 & 2.0010 & 2.0021 & -0.0169 & -0.0062 & 0.0476 & 2.5395 \\
\hline & 100 & 4.1169 & 4.1164 & 4.1169 & 4.1207 & -0.0137 & -0.0022 & 0.0917 & 2.9438 \\
\hline & 105 & 7.1841 & 7.1837 & 7.1840 & 7.1853 & -0.0052 & -0.0018 & 0.0171 & 2.7010 \\
\hline & 110 & 11.0473 & 11.0473 & 11.0472 & 11.0449 & 0.0003 & -0.0010 & -0.0218 & 2.0293 \\
\hline \multirow[t]{5}{*}{60} & 90 & 1.9061 & 1.9061 & 1.9061 & 1.9064 & -0.0027 & 0.0017 & 0.0140 & 4.0187 \\
\hline & 95 & 3.5125 & 3.5118 & 3.5133 & 3.5127 & -0.0196 & 0.0222 & 0.0038 & 5.0139 \\
\hline & 100 & 5.8083 & 5.8074 & 5.8095 & 5.8084 & -0.0163 & 0.0199 & 0.0012 & 5.4663 \\
\hline & 105 & 8.7862 & 8.7855 & 8.7871 & 8.7864 & -0.0085 & 0.0097 & 0.0015 & 5.3017 \\
\hline & 110 & 12.3644 & 12.3642 & 12.3646 & 12.3646 & -0.0016 & 0.0015 & 0.0022 & 4.6523 \\
\hline \multirow[t]{5}{*}{90} & 90 & 2.8985 & 2.8977 & 2.8990 & 2.8988 & 0.0291 & 0.0155 & 0.0084 & 6.0747 \\
\hline & 95 & 4.7109 & 4.7092 & 4.7128 & 4.7105 & 0.0378 & 0.0390 & -0.0087 & 7.0986 \\
\hline & 100 & 7.1015 & 7.0994 & 7.1040 & 7.1008 & 0.0304 & 0.0346 & -0.0099 & 7.5819 \\
\hline & 105 & 10.0522 & 10.0503 & 10.0542 & 10.0518 & 0.0190 & 0.0198 & -0.0046 & 7.4915 \\
\hline & 110 & 13.5044 & 13.5033 & 13.5052 & 13.5046 & 0.0085 & 0.0059 & 0.0010 & 6.9242 \\
\hline \multirow[t]{5}{*}{120} & 90 & 3.7858 & 3.7841 & 3.7873 & 3.7860 & -0.0433 & 0.0410 & 0.0056 & 7.8285 \\
\hline & 95 & 5.7337 & 5.7309 & 5.7372 & 5.7329 & -0.0491 & 0.0606 & -0.0143 & 8.8537 \\
\hline & 100 & 8.1892 & 8.1859 & 8.1935 & 8.1880 & -0.0401 & 0.0523 & -0.0154 & 9.3602 \\
\hline & 105 & 11.1311 & 11.1281 & 11.1348 & 11.1302 & -0.0269 & 0.0334 & -0.0081 & 9.3346 \\
\hline & 110 & 14.5125 & 14.5104 & 14.5147 & 14.5125 & -0.0144 & 0.0148 & 0.0000 & 8.8543 \\
\hline \multirow[t]{5}{*}{180} & 90 & 5.3378 & 5.3346 & 5.3424 & 5.3375 & -0.0601 & 0.0868 & -0.0052 & 10.6045 \\
\hline & 95 & 7.4645 & 7.4598 & 7.4717 & 7.4625 & -0.0628 & 0.0963 & -0.0274 & 11.6077 \\
\hline & 100 & 10.0110 & 10.0057 & 10.0192 & 10.0082 & -0.0528 & 0.0824 & -0.0275 & 12.1468 \\
\hline & 105 & 12.9552 & 12.9502 & 12.9628 & 12.9530 & -0.0384 & 0.0590 & -0.0171 & 12.2263 \\
\hline & 110 & 16.2621 & 16.2582 & 16.2678 & 16.2614 & -0.0239 & 0.0351 & -0.0044 & 11.9015 \\
\hline \multirow[t]{5}{*}{252} & 90 & 6.9387 & 6.9328 & 6.9458 & 6.9361 & -0.0852 & 0.1024 & -0.0382 & 13.0138 \\
\hline & 95 & 9.2060 & 9.1984 & 9.2158 & 9.2009 & -0.0822 & 0.1065 & -0.0547 & 13.9836 \\
\hline & 100 & 11.8297 & 11.8214 & 11.8406 & 11.8237 & -0.0699 & 0.0924 & -0.0506 & 14.5487 \\
\hline & 105 & 14.7891 & 14.7811 & 14.7995 & 14.7837 & -0.0542 & 0.0702 & -0.0363 & 14.7208 \\
\hline & 110 & 18.0562 & 18.0493 & 18.0646 & 18.0527 & -0.0383 & 0.0469 & -0.0193 & 14.5415 \\
\hline \multirow[t]{5}{*}{504} & 90 & 11.3404 & 11.3323 & 11.3521 & 11.3366 & -0.0715 & 0.1031 & -0.0329 & 17.3351 \\
\hline & 95 & 13.8865 & 13.8771 & 13.9001 & 13.8812 & -0.0674 & 0.0982 & -0.0380 & 18.2109 \\
\hline & 100 & 16.6835 & 16.6736 & 16.6980 & 16.6776 & -0.0595 & 0.0871 & -0.0354 & 18.8137 \\
\hline & 105 & 19.7148 & 19.7049 & 19.7292 & 19.7092 & -0.0501 & 0.0730 & -0.0284 & 19.1580 \\
\hline & 110 & 22.9623 & 22.9531 & 22.9757 & 22.9579 & -0.0402 & 0.0581 & -0.0195 & 19.2664 \\
\hline
\end{tabular}

Table 2.3: $P_{m c}$ Monte Carlo put prices computed by $N=10^{6}$ simulations; $\widetilde{P}^{g d(i)}$ GARCH diffusion put prices approximated by (14) truncated up to order $i$-th, for $i=2,3,4 ; e_{p}(i) \%=100\left(\widetilde{P}^{g d(i)}-P_{m c}\right) / P_{m c}$; $e_{m c}$ Monte Carlo standard error. Model parameters: $S_{0}=100, r=d=0 ; d V=(0.18-2 V) d t+$ $0.8 V d W, V_{0}=0.18 / 2$. 


\begin{tabular}{|c|c|c|c|c|c|c|c|c|c|}
\hline$T$ & $K$ & $P_{m c}$ & $\widetilde{P}^{g d(2)}$ & $\widetilde{P}^{g d(3)}$ & $\widetilde{P}^{g d(4)}$ & $e_{p}(2) \%$ & $e_{p}(3) \%$ & $e_{p}(4) \%$ & $e_{m c} \times 10^{4}$ \\
\hline \multirow[t]{5}{*}{30} & 90 & 0.7903 & 0.7905 & 0.7894 & 0.6572 & 0.0265 & -0.1032 & -16.8318 & 2.5217 \\
\hline & 95 & 1.9927 & 1.9913 & 1.9924 & 2.0512 & -0.0715 & -0.0132 & 2.9359 & 3.8020 \\
\hline & 100 & 4.1040 & 4.1018 & 4.1043 & 4.3154 & -0.0542 & 0.0078 & 5.1510 & 4.4035 \\
\hline & 105 & 7.1749 & 7.1733 & 7.1746 & 7.2487 & -0.0218 & -0.0032 & 1.0284 & 4.0433 \\
\hline & 110 & 11.0459 & 11.0458 & 11.0450 & 10.9197 & -0.0008 & -0.0080 & -1.1422 & 3.0517 \\
\hline \multirow[t]{5}{*}{60} & 90 & 1.8942 & 1.8930 & 1.8934 & 1.9000 & -0.0653 & -0.0395 & 0.3078 & 6.0288 \\
\hline & 95 & 3.4865 & 3.4821 & 3.4906 & 3.4725 & -0.1242 & 0.1182 & -0.4017 & 7.4909 \\
\hline & 100 & 5.7761 & 5.7705 & 5.7826 & 5.7520 & -0.0970 & 0.1131 & -0.4162 & 8.1602 \\
\hline & 105 & 8.7583 & 8.7537 & 8.7629 & 8.7427 & -0.0532 & 0.0518 & -0.1780 & 7.9202 \\
\hline & 110 & 12.3481 & 12.3461 & 12.3483 & 12.3508 & -0.0167 & 0.0011 & 0.0212 & 6.9710 \\
\hline \multirow[t]{5}{*}{90} & 90 & 2.8713 & 2.8658 & 2.8737 & 2.8718 & -0.1896 & 0.0855 & 0.0192 & 9.0975 \\
\hline & 95 & 4.6673 & 4.6570 & 4.6791 & 4.6584 & -0.2203 & 0.2544 & -0.1909 & 10.5933 \\
\hline & 100 & 7.0509 & 7.0387 & 7.0669 & 7.0377 & -0.1735 & 0.2268 & -0.1869 & 11.3051 \\
\hline & 105 & 10.0057 & 9.9948 & 10.0186 & 9.9960 & -0.1096 & 0.1283 & -0.0973 & 11.1786 \\
\hline & 110 & 13.4706 & 13.4635 & 13.4754 & 13.4692 & -0.0530 & 0.0353 & -0.0104 & 10.3598 \\
\hline \multirow[t]{5}{*}{120} & 90 & 3.7432 & 3.7322 & 3.7529 & 3.7371 & -0.2931 & 0.2592 & -0.1610 & 11.7084 \\
\hline & 95 & 5.6738 & 5.6565 & 5.6972 & 5.6468 & -0.3051 & 0.4126 & -0.4754 & 13.1994 \\
\hline & 100 & 8.1220 & 8.1021 & 8.1511 & 8.0861 & -0.2447 & 0.3588 & -0.4416 & 13.9428 \\
\hline & 105 & 11.0676 & 11.0492 & 11.0927 & 11.0385 & -0.1662 & 0.2267 & -0.2631 & 13.9152 \\
\hline & 110 & 14.4615 & 14.4479 & 14.4753 & 14.4502 & -0.0940 & 0.0955 & -0.0782 & 13.2316 \\
\hline \multirow[t]{5}{*}{180} & 90 & 5.2682 & 5.2445 & 5.3010 & 5.2279 & -0.4486 & 0.6238 & -0.7644 & 15.8534 \\
\hline & 95 & 7.3776 & 7.3456 & 7.4311 & 7.2935 & -0.4345 & 0.7247 & -1.1400 & 17.3052 \\
\hline & 100 & 9.9165 & 9.8810 & 9.9784 & 9.8146 & -0.3583 & 0.6242 & -1.0280 & 18.0949 \\
\hline & 105 & 12.8633 & 12.8294 & 12.9202 & 12.7734 & -0.2640 & 0.4422 & -0.6989 & 18.2261 \\
\hline & 110 & 16.1813 & 16.1533 & 16.2224 & 16.1268 & -0.1733 & 0.2536 & -0.3372 & 17.7801 \\
\hline \multirow[t]{5}{*}{252} & 90 & 6.8458 & 6.8054 & 6.9104 & 6.7150 & -0.5902 & 0.9427 & -1.9103 & 19.4864 \\
\hline & 95 & 9.0969 & 9.0469 & 9.1869 & 8.8898 & -0.5504 & 0.9895 & -2.2772 & 20.8891 \\
\hline & 100 & 11.7133 & 11.6590 & 11.8138 & 11.4752 & -0.4630 & 0.8583 & -2.0329 & 21.7182 \\
\hline & 105 & 14.6740 & 14.6211 & 14.7694 & 14.4538 & -0.3606 & 0.6502 & -1.5008 & 21.9888 \\
\hline & 110 & 17.9501 & 17.9033 & 18.0272 & 17.7879 & -0.2607 & 0.4297 & -0.9034 & 21.7612 \\
\hline \multirow[t]{5}{*}{504} & 90 & 11.2078 & 11.1406 & 11.3528 & 10.5356 & -0.5990 & 1.2940 & -5.4470 & 28.4149 \\
\hline & 95 & 13.7415 & 13.6659 & 13.9123 & 12.9098 & -0.5502 & 1.2428 & -6.0527 & 27.5161 \\
\hline & 100 & 16.5319 & 16.4522 & 16.7143 & 15.6314 & -0.4823 & 1.1035 & -5.9976 & 26.2295 \\
\hline & 105 & 19.5621 & 19.4824 & 19.7423 & 18.6831 & -0.4074 & 0.9214 & -4.4932 & 28.9460 \\
\hline & 110 & 22.8135 & 22.7376 & 22.9793 & 22.0352 & -0.3327 & 0.7267 & -3.4116 & 29.1419 \\
\hline
\end{tabular}

Table 2.4: $P_{m c}$ Monte Carlo put prices computed by $N=10^{6}$ simulations; $\widetilde{P}^{g d(i)}$ GARCH diffusion put prices approximated by (14) truncated up to order $i$-th, for $i=2,3,4 ; e_{p}(i) \%=100\left(\widetilde{P}^{g d(i)}-P_{m c}\right) / P_{m c}$; $e_{m c}$ Monte Carlo standard error. Model parameters: $S_{0}=100, r=d=0 ; d V=(0.18-2 V) d t+$ $1.2 V d W, V_{0}=0.18 / 2$. 


\begin{tabular}{|c|c|c|c|c|c|c|c|c|c|}
\hline$T$ & $K$ & $P_{m c}$ & $\widetilde{P}^{g d(2)}$ & $\widetilde{P}^{g d(3)}$ & $\widetilde{P}^{g d(4)}$ & $e_{p}(2) \%$ & $e_{p}(3) \%$ & $e_{p}(4) \%$ & $e_{m c} \times 10^{4}$ \\
\hline \multirow[t]{5}{*}{30} & 90 & 0.0416 & 0.0419 & 0.0418 & 0.0417 & 0.8998 & 0.4710 & 0.2776 & 0.2796 \\
\hline & 95 & 0.4270 & 0.4273 & 0.4269 & 0.4273 & 0.0629 & -0.0227 & 0.0759 & 1.2305 \\
\hline & 100 & 2.0541 & 2.0536 & 2.0546 & 2.0539 & -0.0230 & 0.0235 & -0.0094 & 2.0376 \\
\hline & 105 & 5.4911 & 5.4913 & 5.4910 & 5.4914 & 0.0043 & -0.0016 & 0.0059 & 1.3532 \\
\hline & 110 & 10.0742 & 10.0747 & 10.0744 & 10.0744 & 0.0051 & 0.0016 & 0.0019 & 0.4322 \\
\hline \multirow[t]{5}{*}{60} & 90 & 0.2403 & 0.2415 & 0.2395 & 0.2409 & 0.5118 & -0.3186 & 0.2691 & 1.2698 \\
\hline & 95 & 1.0088 & 1.0085 & 1.0088 & 1.0093 & -0.0271 & -0.0013 & 0.0489 & 2.7060 \\
\hline & 100 & 2.8976 & 2.8961 & 2.8995 & 2.8968 & -0.0534 & 0.0661 & -0.0294 & 3.5178 \\
\hline & 105 & 6.1229 & 6.1225 & 6.1230 & 6.1233 & -0.0064 & 0.0019 & 0.0071 & 2.9091 \\
\hline & 110 & 10.3537 & 10.3550 & 10.3527 & 10.3546 & 0.0118 & -0.0097 & 0.0088 & 1.6629 \\
\hline \multirow[t]{5}{*}{90} & 90 & 0.5059 & 0.5073 & 0.5040 & 0.5076 & 0.2868 & -0.3736 & 0.3476 & 2.2925 \\
\hline & 95 & 1.5236 & 1.5223 & 1.5243 & 1.5238 & -0.0811 & 0.0482 & 0.0119 & 3.8280 \\
\hline & 100 & 3.5450 & 3.5421 & 3.5484 & 3.5429 & -0.0808 & 0.0953 & -0.0581 & 4.5905 \\
\hline & 105 & 6.6746 & 6.6732 & 6.6756 & 6.6747 & -0.0215 & 0.0146 & 0.0004 & 4.0842 \\
\hline & 110 & 10.6943 & 10.6955 & 10.6924 & 10.6963 & 0.0110 & -0.0180 & 0.0186 & 2.8388 \\
\hline \multirow[t]{5}{*}{120} & 90 & 0.7895 & 0.7907 & 0.7873 & 0.7923 & 0.1612 & -0.2753 & 0.3633 & 3.1594 \\
\hline & 95 & 1.9843 & 1.9823 & 1.9862 & 1.9839 & -0.1032 & 0.0935 & -0.0194 & 4.6713 \\
\hline & 100 & 4.0917 & 4.0879 & 4.0965 & 4.0884 & -0.0926 & 0.1164 & -0.0804 & 5.3827 \\
\hline & 105 & 7.1657 & 7.1634 & 7.1679 & 7.1651 & -0.0321 & 0.0305 & -0.0089 & 4.9653 \\
\hline & 110 & 11.0435 & 11.0443 & 11.0416 & 11.0464 & 0.0072 & -0.0170 & 0.0262 & 3.8040 \\
\hline \multirow[t]{5}{*}{180} & 90 & 1.3561 & 1.3566 & 1.3550 & 1.3596 & 0.0413 & -0.0806 & 0.2637 & 4.4457 \\
\hline & 95 & 2.7925 & 2.7896 & 2.7964 & 2.7910 & -0.1048 & 0.1395 & -0.0549 & 5.8221 \\
\hline & 100 & 5.0114 & 5.0069 & 5.0181 & 5.0068 & -0.0903 & 0.1319 & -0.0932 & 6.4495 \\
\hline & 105 & 8.0238 & 8.0206 & 8.0281 & 8.0219 & -0.0401 & 0.0539 & -0.0234 & 6.1656 \\
\hline & 110 & 11.7203 & 11.7202 & 11.7200 & 11.7235 & -0.0009 & -0.0026 & 0.0269 & 5.2043 \\
\hline \multirow[t]{5}{*}{252} & 90 & 2.0035 & 2.0028 & 2.0036 & 2.0056 & -0.0340 & 0.0071 & 0.1075 & 5.4574 \\
\hline & 95 & 3.6288 & 3.6251 & 3.6333 & 3.6266 & -0.1024 & 0.1240 & -0.0631 & 6.6719 \\
\hline & 100 & 5.9334 & 5.9284 & 5.9400 & 5.9288 & -0.0845 & 0.1108 & -0.0770 & 7.2297 \\
\hline & 105 & 8.9091 & 8.9051 & 8.9140 & 8.9065 & -0.0451 & 0.0551 & -0.0293 & 7.0508 \\
\hline & 110 & 12.4759 & 12.4745 & 12.4770 & 12.4775 & -0.0114 & 0.0084 & 0.0123 & 6.2867 \\
\hline \multirow[t]{5}{*}{504} & 90 & 3.9613 & 3.9603 & 3.9637 & 3.9655 & -0.0267 & 0.0605 & 0.1053 & 7.0744 \\
\hline & 95 & 5.9352 & 5.9329 & 5.9394 & 5.9446 & -0.0390 & 0.0710 & 0.1581 & 7.9420 \\
\hline & 100 & 8.4032 & 8.4004 & 8.4082 & 8.4148 & -0.0335 & 0.0592 & 0.1378 & 8.3767 \\
\hline & 105 & 11.3436 & 11.3412 & 11.3481 & 11.3537 & -0.0218 & 0.0396 & 0.0886 & 8.3715 \\
\hline & 110 & 14.7118 & 14.7103 & 14.7148 & 14.7175 & -0.0099 & 0.0208 & -0.0393 & 7.9875 \\
\hline
\end{tabular}

Table 2.5: $P_{m c}$ Monte Carlo put prices computed by $N=10^{6}$ simulations; $\widetilde{P}^{g d(i)}$ GARCH diffusion put prices approximated by (14) truncated up to order $i$-th, for $i=2,3,4 ; e_{p}(i) \%=100\left(\widetilde{P}^{g d(i)}-P_{m c}\right) / P_{m c}$; $e_{m c}$ Monte Carlo standard error. Model parameters: $S_{0}=100, r=d=0 ; d V=(0.09-4 V) d t+$ $1.2 V d W, V_{0}=0.09 / 4$. 

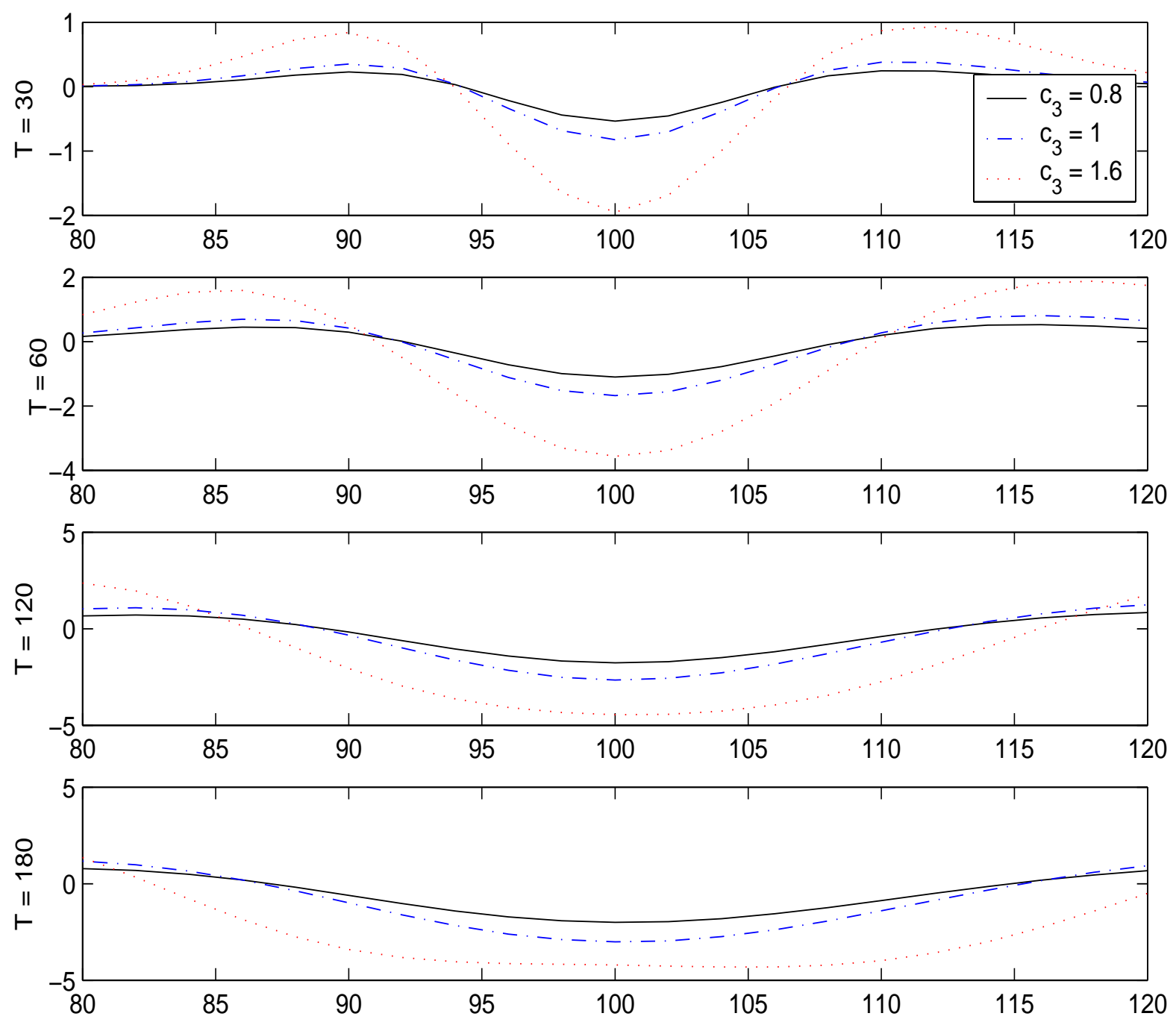

Figure 2.1: $c_{3}$ effect. Percent Bias $=100\left(\widetilde{C}^{g d}-C_{b s}\right)$ for different maturities and parameter values of $c_{3}$, when $S_{0}=100, r=d=0 ; d V=(0.09-4 V) d t+c_{3} V d W, V_{0}=0.0225$. 

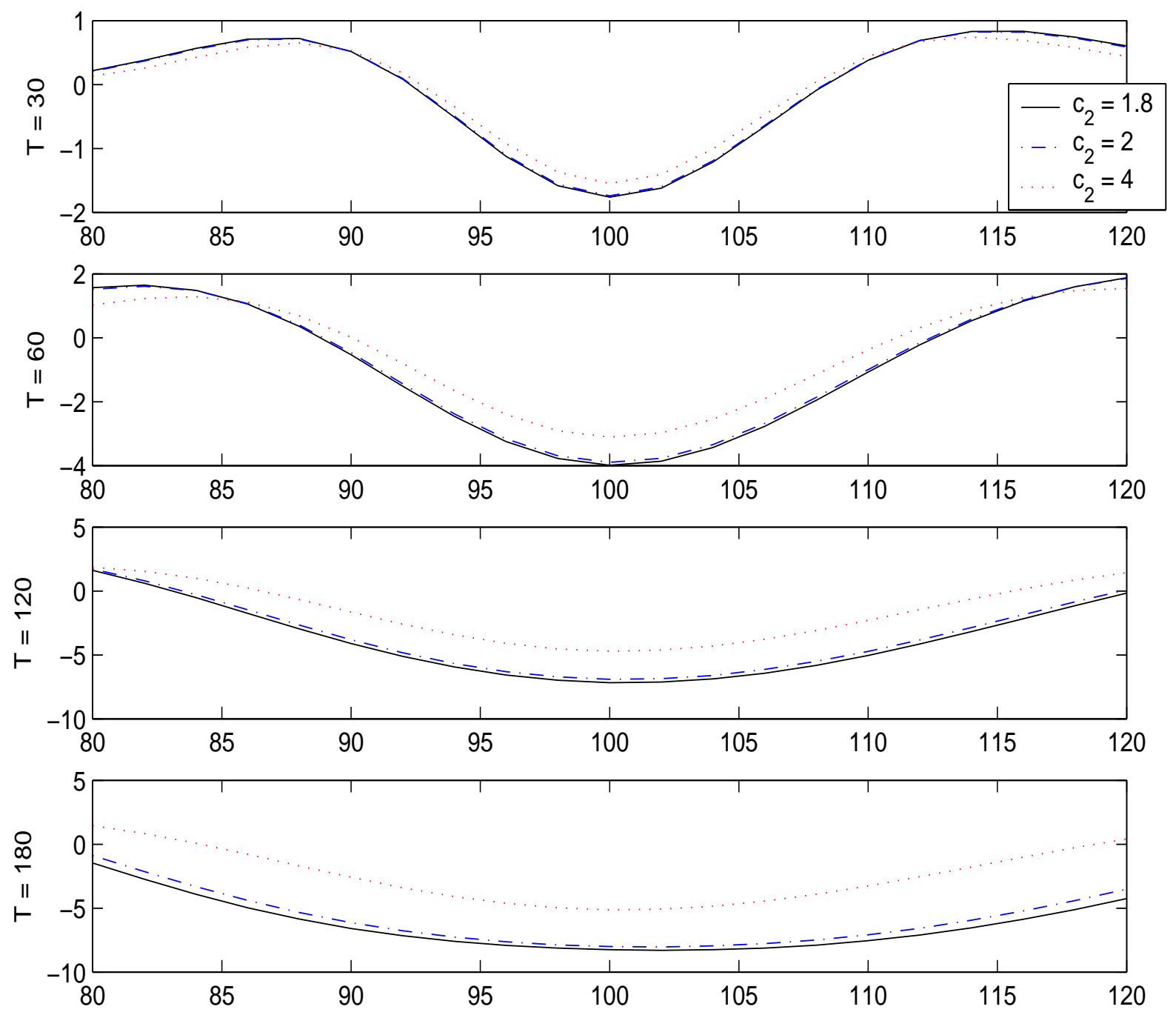

Figure 2.2: $c_{2}^{*}$ effect. Percent Bias $=100\left(\widetilde{C}^{g d}-C_{b s}\right)$ for different maturities and parameter values of $c_{2}^{*}$, when $S_{0}=100, r=d=0 ; d V=\left(0.9-c_{2}^{*} V\right) d t+1.2 V d W, V_{0}=0.0225$. 

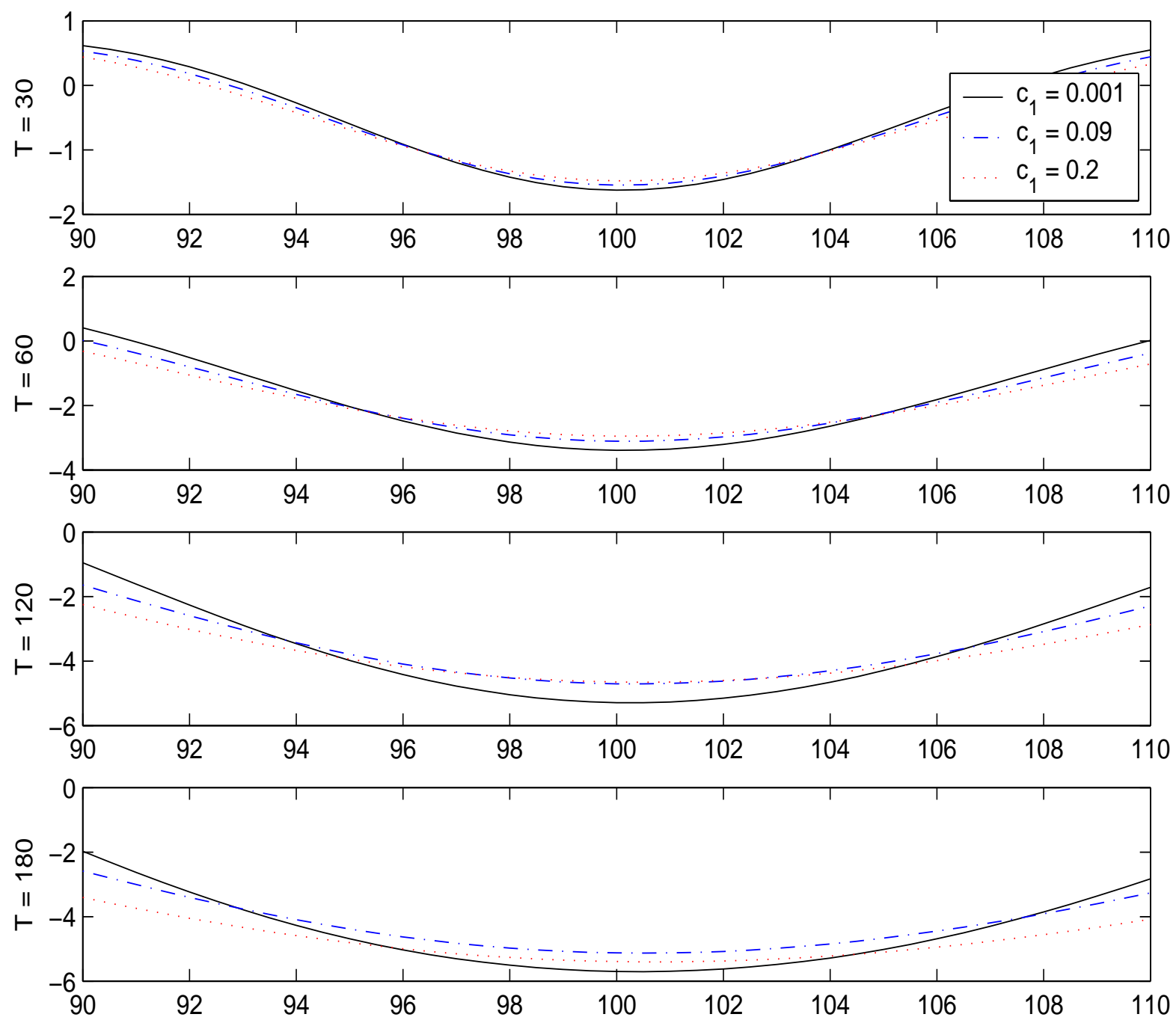

Figure 2.3: $c_{1}$ effect. Percent Bias $=100\left(\widetilde{C}^{g d}-C_{b s}\right)$ for different maturities and parameter values of $c_{1}$, when $S_{0}=100, r=d=0 ; d V=\left(c_{1}-4 V\right) d t+1.2 V d W, V_{0}=0.0225$. 

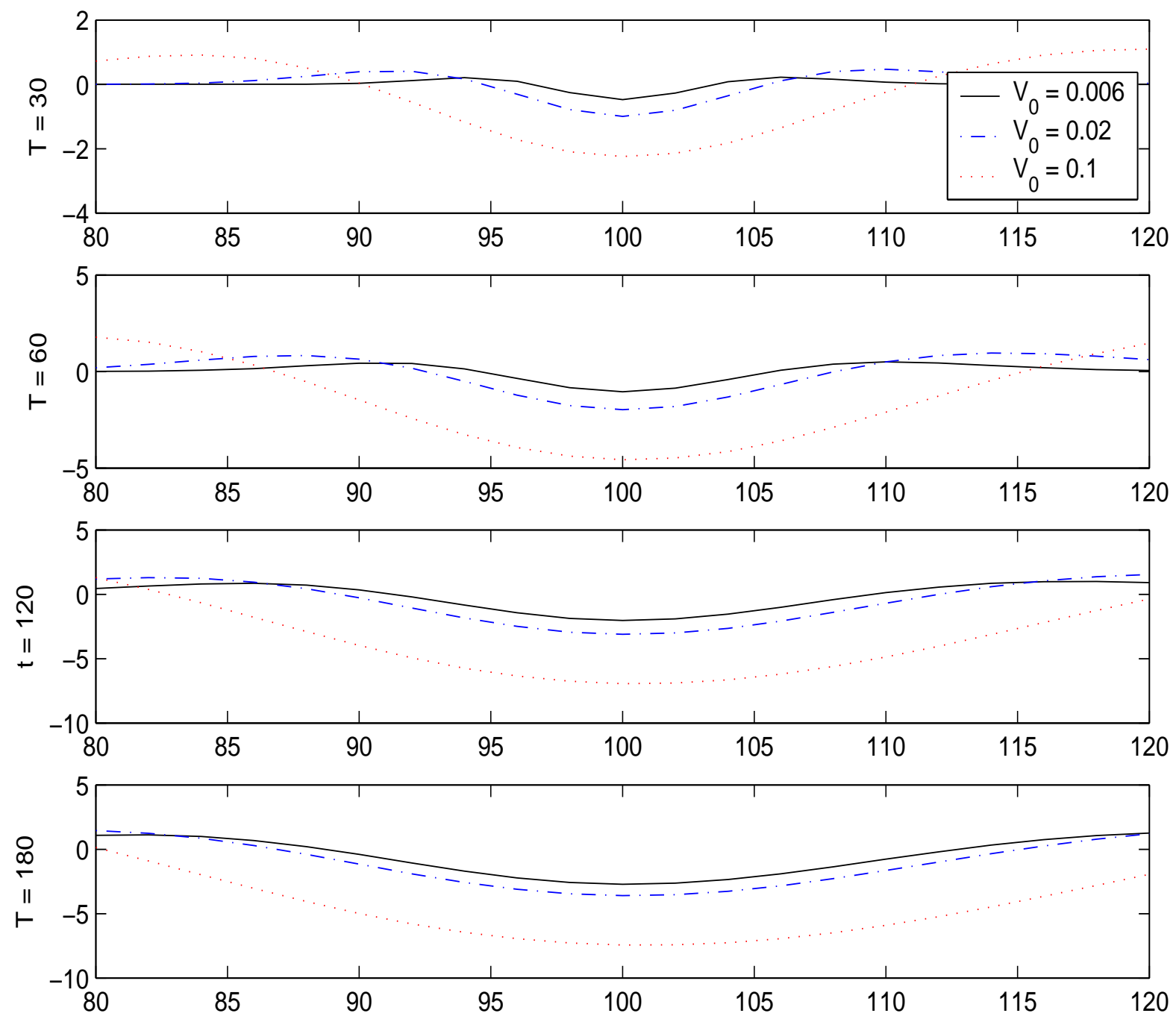

Figure 2.4: $V_{0}$ effect. Percent Bias $=100\left(\widetilde{C}^{g d}-C_{b s}\right)$ for different maturities and initial variance $V_{0}$, when $S_{0}=100, r=d=0 ; d V=(0.09-4 V) d t+1.2 V d W$. 

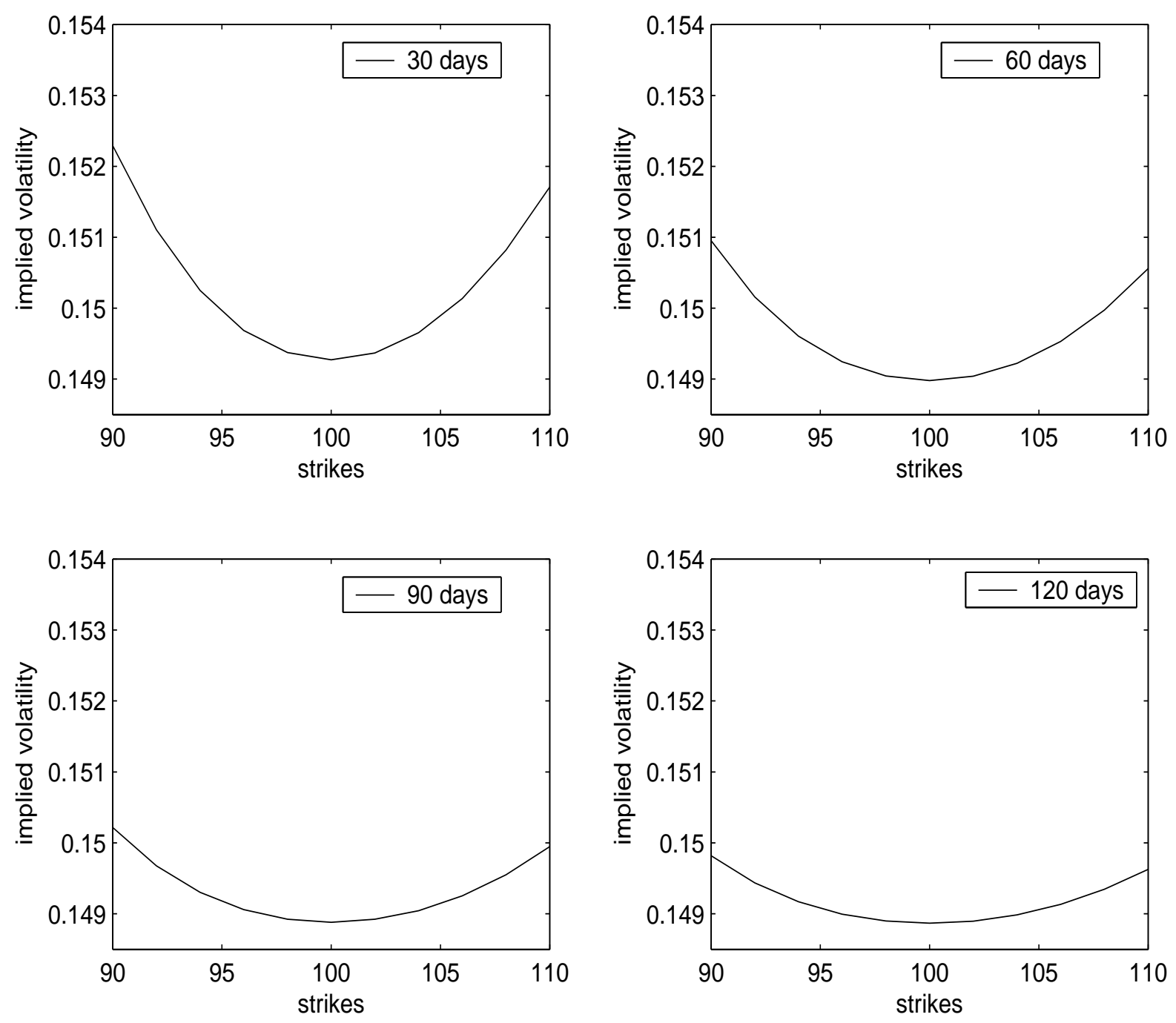

Figure 2.5: Volatility smiles for maturities of 30,60, 90 and 120 days and the parameter choice $S_{0}=100$, $r=d=0 ; d V=(0.09-4 V) d t+1.2 V d W, V_{0}=0.0225$, as in Table 2.5 . 


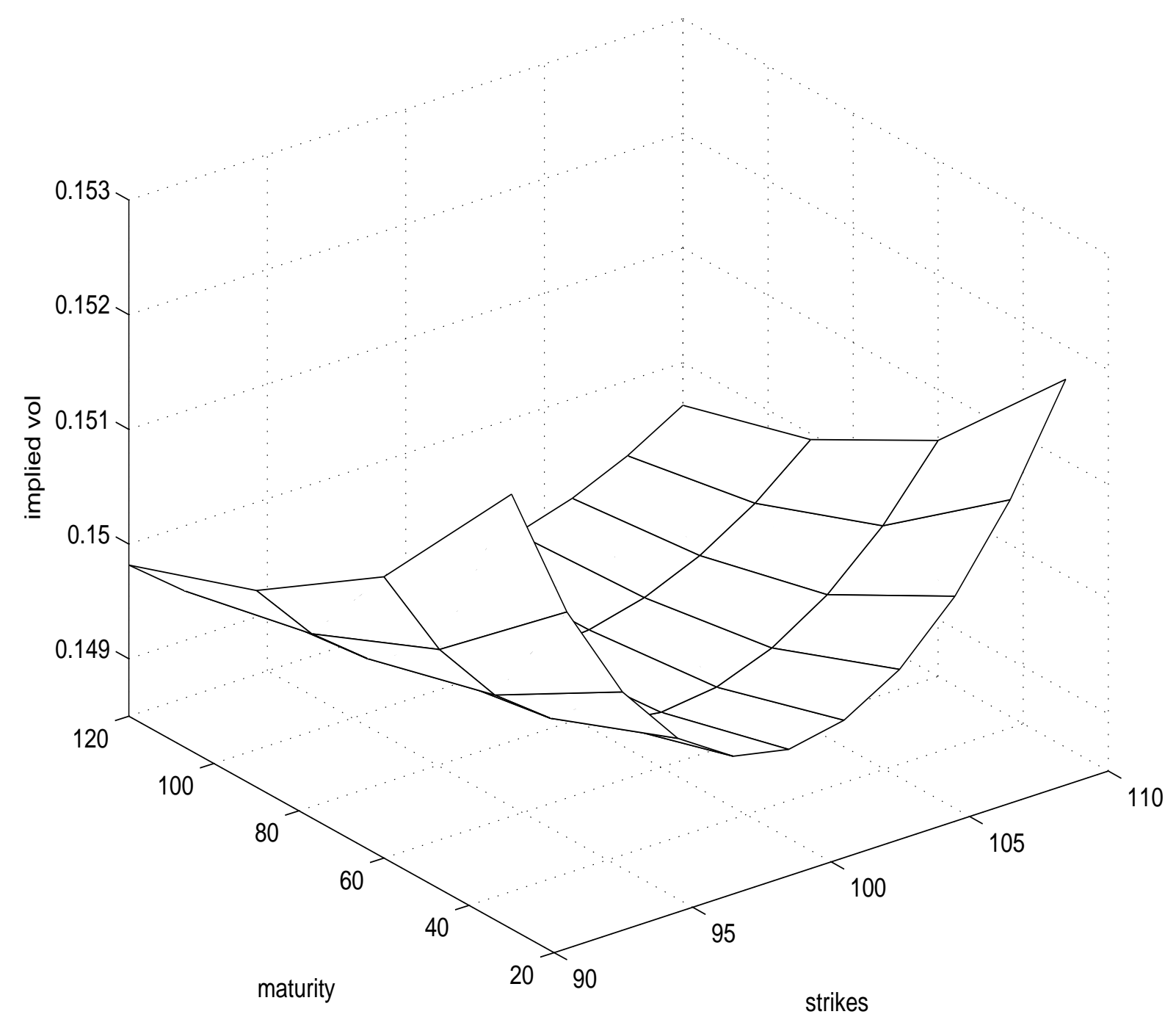

Figure 2.6: Volatility Surface for maturities $T \in[30,120]$ days, strikes $K \in[90,110]$ and the parameter choice $S_{0}=100, r=d=0 ; d V=(0.09-4 V) d t+1.2 V d W, V_{0}=0.0225$, as in Table 2.5. 


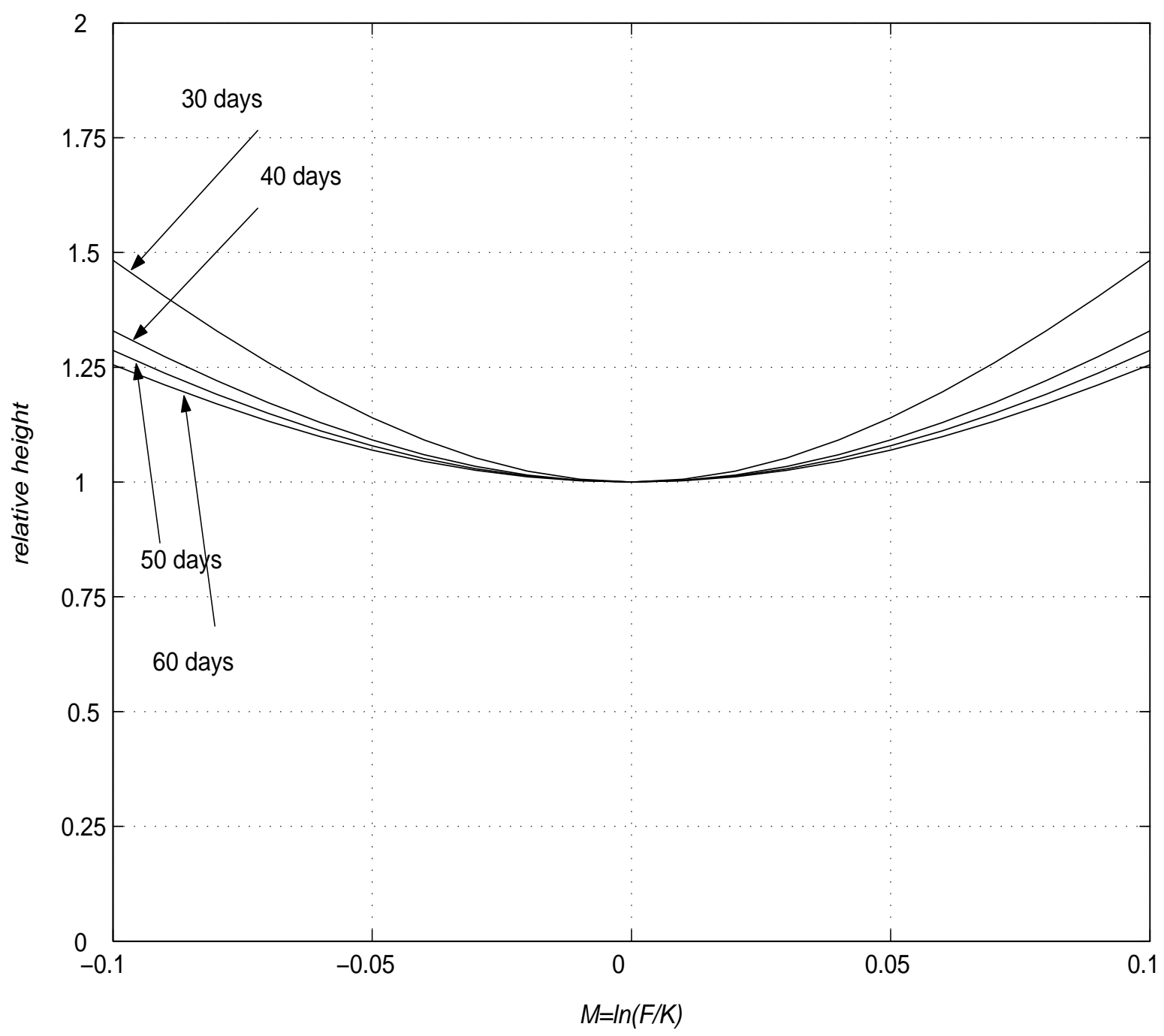

Figure 2.7: Relative height of volatility smiles of Mark-Dollar call options (1985-1992) 


\section{Chapter 3}

\section{Inference based on Nelson's Theory}

Inference on continuous time parameters of stochastic volatility models is an important issue in financial econometrics. The intractable likelihood function and the unobservable variance process prevent simple and efficient estimation procedures for such models. Several estimation methods have been proposed $^{1}$, such as the simulation based method of moments of Duffie and Singleton (1993) or the Bayesian Markov chain Monte Carlo methods of Jones (2003). Such procedures are rather difficult to implement and computationally demanding.

Nelson (1990) proposed a simple estimator for the GARCH diffusion model. Under some moment conditions, Nelson shows that the discrete time $\operatorname{GARCH}(1,1)-\mathrm{M}$ model (cf. Engle and Bollerslev (1986)) converges in distribution to the GARCH diffusion model (1.7)-(1.8). Hence, the inference on continuous time parameters reduces to inference on discrete time $\operatorname{GARCH}(1,1)-\mathrm{M}$ parameters. As GARCH(1,1)-M model can be easily estimated, this procedure has been advocated by many authors (see, for instance, Engle and Lee (1996) and Lewis (2000)) to infer the continuous time parameters. Moreover, in an empirical study, Engle and Lee (1996) show that the estimates of the GARCH diffusion model based on the Nelson's theory and on the indirect inference method are quite close. However,

\footnotetext{
${ }^{1}$ A survey on stochastic volatility models including the estimation problem is given, for instance, by Jiang (1998).
} 
to our knowledge, the accuracy of the continuous time parameter estimates obtained by the discrete time parameter estimates has not been adequately verified.

In section 3.1 we investigate by Monte Carlo simulations the Nelson's theory and the convergence, under proper conditions, of the stochastic difference equations (3.1) to the stochastic differential equations (1.7)-(1.8) when the time interval between two observations goes to zero.

\subsection{Simple Estimators for the GARCH Diffusion Model}

The discrete time GARCH(1,1)-M model is

$$
\begin{aligned}
Y_{k h} & =Y_{(k-1) h}+\left[\mu-\frac{\sigma_{k h}^{2}}{2}\right] h+\sigma_{k h} \sqrt{h} Z_{k h} \\
\sigma_{(k+1) h}^{2} & =w^{(h)}+\beta^{(h)} \sigma_{k h}^{2}+\alpha^{(h)} \sigma_{k h}^{2} Z_{k h}^{2},
\end{aligned}
$$

where $Y_{k h}:=\log \left(S_{k h}\right), k \in \mathbb{N}, Z_{k h} \sim$ i.i.d. $\mathcal{N}(0,1)$ and $\sigma_{k h}^{2}$ is the conditional variance of $Y_{k h}$ $Y_{(k-1) h}$ given the $\sigma$-algebra $M_{k h}$ generated by $\left\{Y_{0}, \ldots, Y_{(k-1) h}\right\}$ and $\left\{\sigma_{0}^{2}, \ldots, \sigma_{k h}^{2}\right\}$. Under some moment conditions, Nelson showed that, the sequence of continuous time version of (3.1) converges in distribution to the continuous time process (1.7)-(1.8) if $h$ tends to zero. We show in Appendix A.4 that up to $o(h)$, the procedure yields the following relation between continuous and discrete time parameters

$$
c_{1}=w^{(h)} / h, \quad c_{2}=1-\beta^{(h)}-\alpha^{(h)}, \quad c_{3}=\alpha^{(h)} \sqrt{2 / h}
$$

cf. Nelson (1990), pp. 15-18. Then, Maximum Likelihood estimates of the model (3.1) allow to infer the values for $c_{1}, c_{2}$ and $c_{3}$. Moreover, the theory predicts that when $h$ goes to zero the inference results should be more accurate. Using an Euler scheme ${ }^{2}$, we simulate 1,000 sample path realizations of the

\footnotetext{
${ }^{2}$ The Euler scheme for equations (1.13) used here is $\log \left(S_{i}\right)=\log \left(S_{i-1}\right)+\left[\mu-0.5 V_{i-1}\right] \Delta t+\sqrt{V_{i-1} \Delta t} \epsilon_{i}$ and $V_{i}=c_{1} \Delta t+V_{i-1}\left[1-c_{2} \Delta t+c_{3} \sqrt{\Delta t} v_{i}\right]$, where $\epsilon_{i}$ and $v_{i}$ denote independent standard normal random variables and $\Delta t=1 /(360 \times 24)$. The sample path approximation to the diffusion can be made arbitrarily close by increasing the number of equally spaced increments per unit time interval. In this setting, the Milstein scheme is not advisable as
} 
GARCH diffusion process $\left(\log S_{t}, V_{t}\right)_{t \in\left[0, T_{s m p}\right]}$, for the parameter choice $c_{1}=0.18, c_{2}=2, c_{3}=1.2$ (as in Table 2.4) and $T_{s m p}=10,20,40$ years. For each sample path, we estimate the model (3.1) using two-daily $(h=2 / 360)$, daily $(h=1 / 360)$ and two-hourly $(h=1 / 3,000)$ log-returns and we infer the continuous time parameters by formulae (3.2). Estimation results are given in Table 3.1; cf. also Figures 3.1, 3.2 and 3.3.

As expected, the higher the sampling frequency, the more accurate the inference results are in terms of biases and root mean squared errors. Obviously, results based on $T_{s m p}=40$ years are more accurate, but such a sample size is unrealistic in empirical studies. However, this simple estimation procedure still provides accurate inference results using $T_{s m p}=20$ years; cf. Figure 3.2. Monte Carlo simulations (not reported here) based on other continuous time parameters lead to similar results. Hence, such a procedure may be used for inference on the GARCH diffusion model parameters. More involved estimation procedures should be required to outperform the above results.

Finally, when $T_{s m p}=40$ years, for two-daily estimates the sample size is large enough to overcome finite sample problems and regard the estimates as asymptotic ${ }^{3}$.

\subsection{Empirical Application}

Using the estimator based on the Nelson's theory, we fit the GARCH diffusion model to daily logreturns of Deutsche mark versus US dollar exchange rates. The data were downloaded from datastream. We consider two sample periods.

The first period ranges from December 1988 to December 2003 for a total of 3,912 observations. Summary statistics of log-returns are presented in the first panel of Table 3.2. The first panel in

multiple stochastic integrals can not be easily expressed in terms of increments of Brownian Motions; see for instance Kloeden and Platen (1999).

${ }^{3}$ We repeat the Monte Carlo study using $T_{s m p}=60$ years and we get similar results. 
Table 3.3 presents the Maximum Likelihood estimates of the GARCH(1,1)-M model with p-values in brackets. The second panel shows the Ljung-Box test statistics for $Z_{t}^{2}$ and $\left|Z_{t}\right|$. Under the null hypothesis of no correlations up to lag $q$, such statistics are chi-square distributed with $q$ degrees of freedom. We set $q=15$ and found no evidence against the null hypothesis. Hence, based on such statistics, the model is tenable. Figure 3.4 shows the daily log-returns (first panel) and the corresponding conditional variances estimated by the $\operatorname{GARCH}(1,1)-\mathrm{M}$ model (second panel). The continuous time parameters of the GARCH diffusion model, obtained by formulae (3.2), are $c_{1}=0.030$, $c_{2}=2.586$ and $c_{3}=0.899$ on annual basis. Hence, the variance process is slow mean reverting (the half life is about 68 trading days) and the volatility is not too large. The unconditional mean of the variance is 0.0116 and the kurtosis 3.56 .

The second sample period ranges from January 1985 to October 1988, for a total of 1,000 observations. Summary statistics of log-returns are given in the second panel of Table 3.2. This data set was used by Engle and Lee (1996) to estimate the GARCH diffusion model (1.7)-(1.8), applying the indirect inference method; cf. Gourieroux et al. (1993). Hence, we can compare estimates for the GARCH diffusion model based on the indirect inference and the Nelson's theory. The Maximum Likelihood estimates of the GARCH(1,1)-M are reported in Table 3.4. Figure 3.5 shows the daily log-returns (first panel) and the conditional variances estimated by the $\operatorname{GARCH}(1,1)-\mathrm{M}$ model (second panel). The continuous time parameter estimates of the GARCH diffusion model are $c_{2}=7, c_{1} / c_{2}=0.023$ and $c_{3}=3.1$ (on anual basis). The corresponding estimates in Engle and Lee (1996) are $c_{2}=4$, $c_{1} / c_{2}=0.020$ and $c_{3}=2.6$. Notwithstanding the small sample data, the accordance for the parameter $c_{3}$ and the long-run mean value $c_{1} / c_{2}$ is quite good. As predicted by Monte Carlo simulations in Section 3.1, using the Nelson's theory the parameter $c_{2}$ is overestimated. 


\begin{tabular}{|c|c|c|c|c|c|c|}
\hline \multirow{2}{*}{} & \multicolumn{2}{|c|}{$c_{1}=0.18$} & \multicolumn{2}{c|}{$c_{2}=2$} & \multicolumn{2}{c|}{$c_{3}=1.2$} \\
\cline { 2 - 7 } & bias & rsme & bias & rsme & bias & rsme \\
\hline 2 days & 0.0779 & 0.1525 & 1.0167 & 2.0361 & -0.1332 & 0.2711 \\
\hline 1 days & 0.0626 & 0.1247 & 0.8340 & 1.7041 & -0.0995 & 0.2043 \\
\hline 2 hours & 0.0396 & 0.0776 & 0.5571 & 1.1631 & -0.0264 & 0.0826 \\
\hline \hline 2 days & 0.0442 & 0.0900 & 0.5814 & 1.2041 & -0.1379 & 0.2185 \\
\hline 1 days & 0.0351 & 0.0721 & 0.4720 & 1.0035 & -0.1003 & 0.1618 \\
\hline 2 hours & 0.0213 & 0.0505 & 0.3086 & 0.7758 & -0.025 & 0.0606 \\
\hline \hline 2 days & 0.0283 & 0.0550 & 0.3531 & 0.7409 & -0.1296 & 0.1718 \\
\hline 1 days & 0.0232 & 0.0465 & 0.2939 & 0.6377 & -0.1003 & 0.1279 \\
\hline 2 hours & 0.0111 & 0.0302 & 0.1480 & 0.4692 & -0.0257 & 0.0470 \\
\hline
\end{tabular}

Table 3.1: Bias RMSE First panel: sample size 10 years; second panel: sample size 20 years; third panel: sample size 40 years

\begin{tabular}{|c|c|c|c|c|}
\hline & mean & variance & kurtosis & iqr \\
\hline Dec.88-Dec.03 & -0.000275 & 0.011 & 4.7 & 0.119 \\
\hline \hline Jan.85-Oct.88 & -0.006590 & 0.016 & 7.4 & 0.143 \\
\hline
\end{tabular}

Table 3.2: Summary statistics (mean, variance, kurtosis and inter quartile range) of DM/US exchange rates daily log-returns.

\begin{tabular}{|c|c|c|c|c|}
\hline & $\mu$ & $\omega$ & $\alpha$ & $\beta$ \\
\hline Estimate & $2.5 \mathrm{e}-006$ & $4.7 \mathrm{e}-007$ & 0.030649 & 0.95909 \\
p-value & 0.8355 & 0.0000 & 0.0000 & 0.0000 \\
\hline \hline Ljung-box test $^{a}$ & \multicolumn{2}{|c|}{$Z_{t}^{2}$} & \multicolumn{2}{|c|}{$\left|Z_{t}\right|$} \\
\hline$\chi^{2}$ & \multicolumn{2}{|c|}{13.189} & \multicolumn{2}{|c|}{16.561} \\
p-value & \multicolumn{2}{|c|}{0.58772} & \multicolumn{2}{c|}{0.34577} \\
\hline
\end{tabular}

Table 3.3: GARCH(1,1)-M estimates for DM/US daily exchange rate from December 1988 to December 2003. $Z_{t}, \mu, \omega, \alpha$ and $\beta$ are defined in equations (3.1). ${ }^{a}$ Ljung-Box test follows $\chi^{2}(15)$ with the 0.05 critical value being 25 . 


\begin{tabular}{|c|c|c|c|c|}
\hline & $\mu$ & $\omega$ & $\alpha$ & $\beta$ \\
\hline Estimate & $-3.6 \mathrm{e}+04$ & $2.5 \mathrm{e}-006$ & 0.13913 & 0.83293 \\
p-value & 0.0811 & 0.0003 & 0.000 & 0.000 \\
\hline \hline Ljung-box test $^{a}$ & \multicolumn{2}{|c|}{$Z_{t}^{2}$} & \multicolumn{2}{|c|}{$\left|Z_{t}\right|$} \\
\hline$\chi^{2}$ & \multicolumn{2}{|c|}{14.977} & \multicolumn{2}{|c|}{11.978} \\
p-value & \multicolumn{2}{|c|}{0.45308} & 0.68066 \\
\hline
\end{tabular}

Table 3.4: GARCH(1,1)-M estimates for DM/US daily exchange rates from January 1985 to October 1988. $Z_{t}, \mu, \omega, \alpha$ and $\beta$ are defined in equations (3.1). ${ }^{a}$ Ljung-Box test follows $\chi^{2}(15)$ with the 0.05 critical value being 25 . 

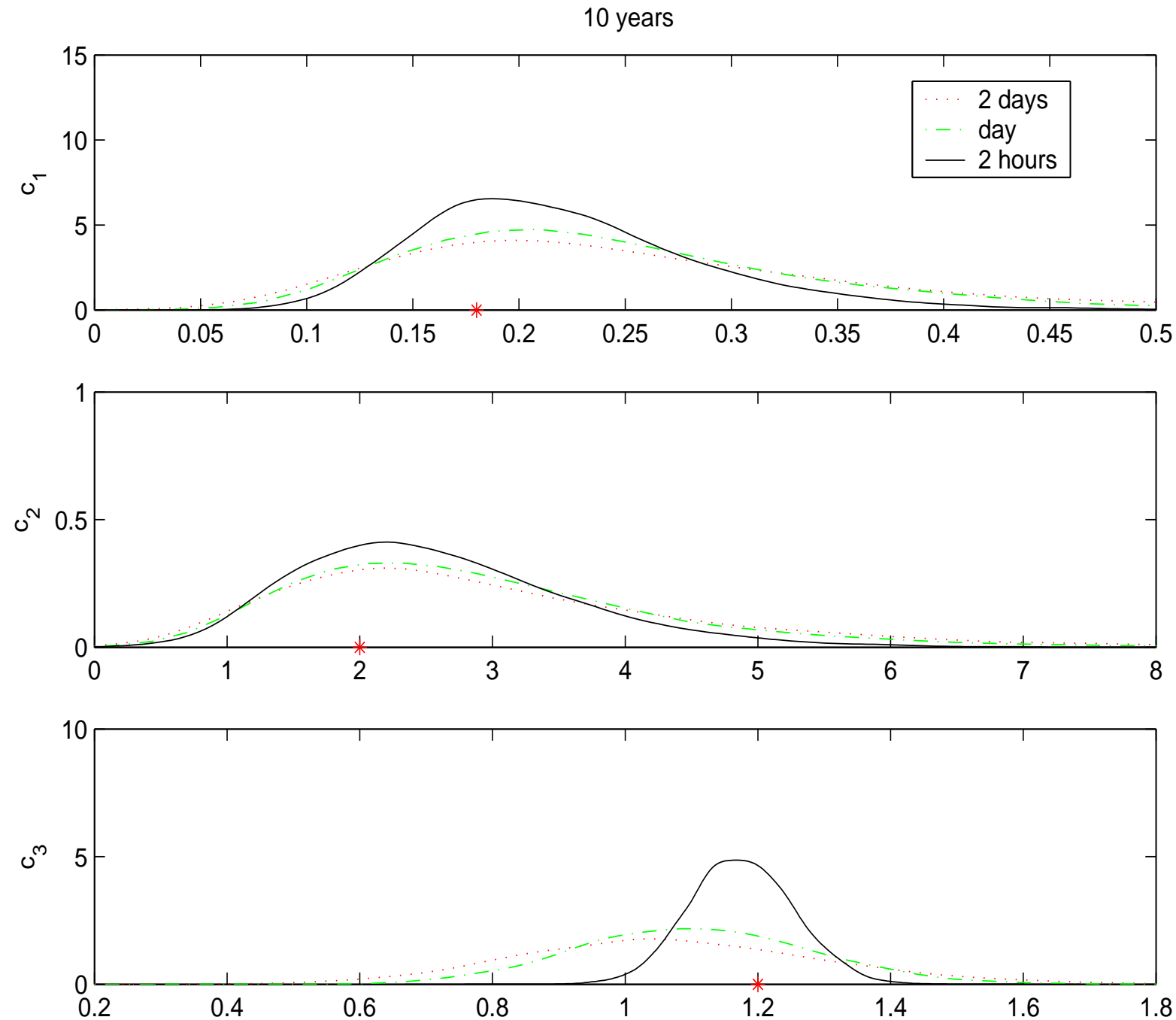

Figure 3.1: Density estimates of the continuous time parameters $c_{1}=0.18, c_{2}=2$ and $c_{3}=1.2$ of the GARCH diffusion model (1.7)-(1.8) inferred by the parameter estimates of the discrete time GARCH-M model (3.1) at two-daily, daily and two-hourly sampling frequencies. Sample size 10 years. 

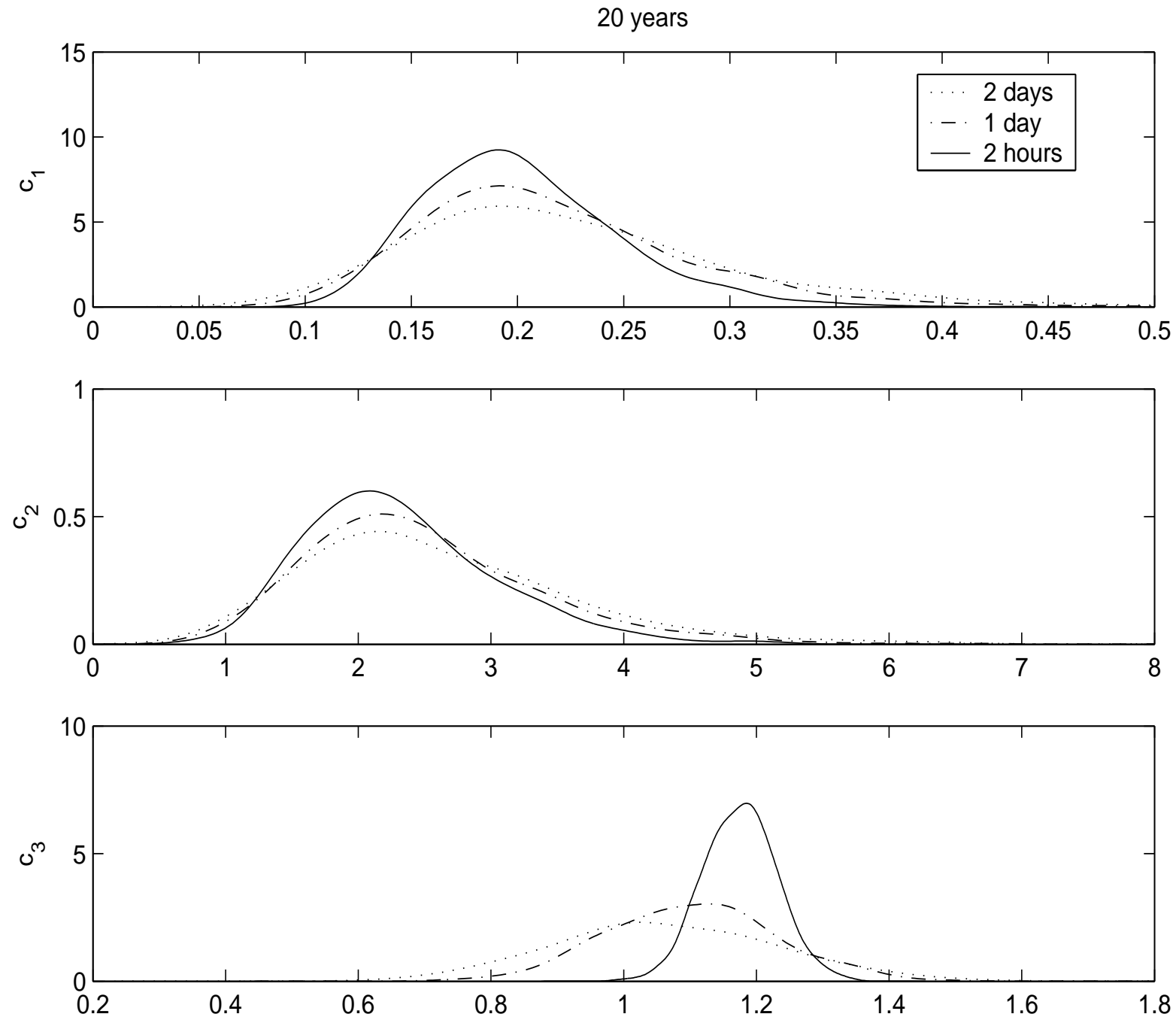

Figure 3.2: Density estimates of the continuous time parameters $c_{1}=0.18, c_{2}=2$ and $c_{3}=1.2$ of the GARCH diffusion model (1.7)-(1.8) inferred by the parameter estimates of the discrete time GARCH-M model (3.1) at two-daily, daily and two-hourly sampling frequencies. Sample size 20 years. 

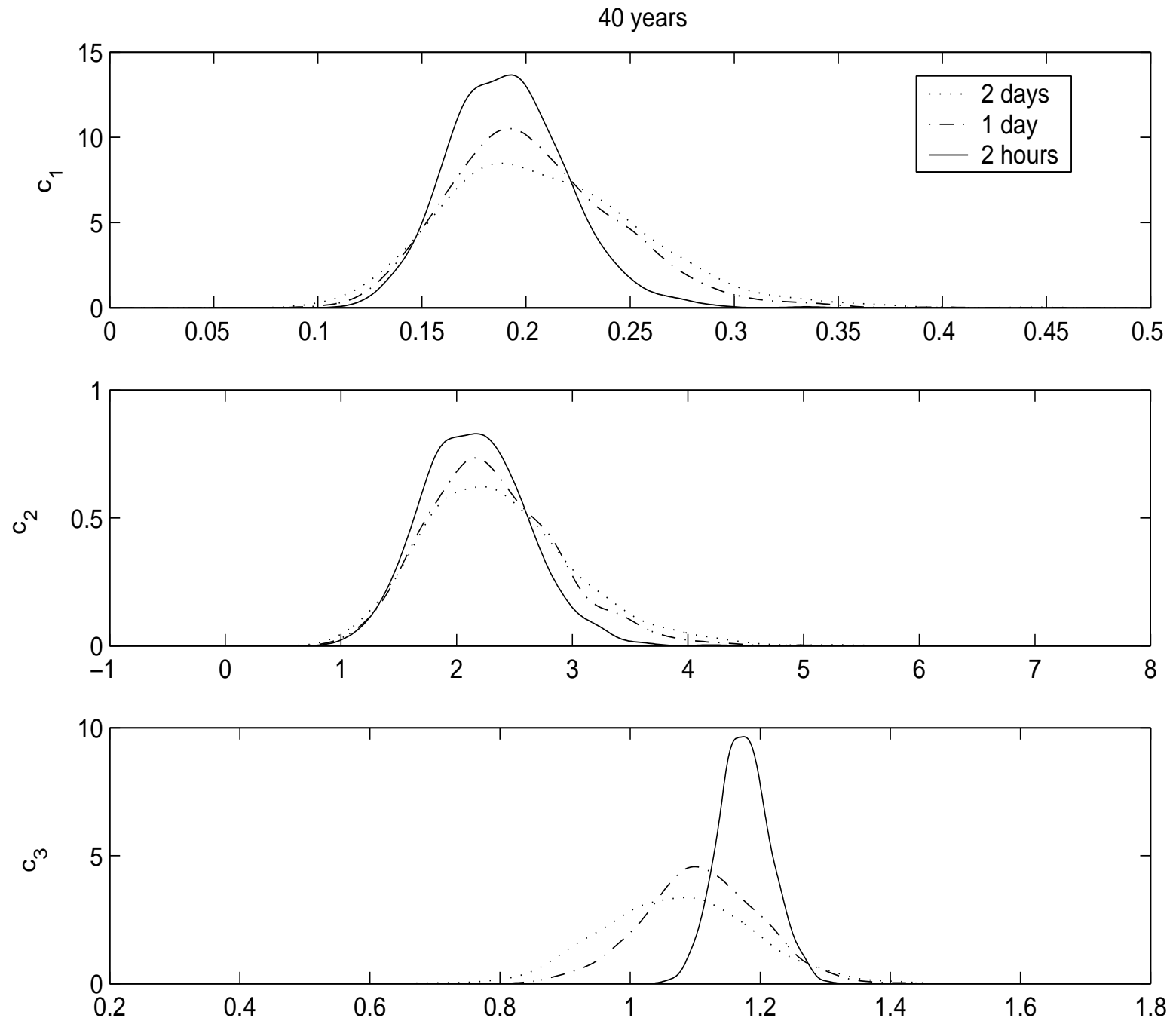

Figure 3.3: Density estimates of the continuous time parameters $c_{1}=0.18, c_{2}=2$ and $c_{3}=1.2$ of the GARCH diffusion model (1.7)-(1.8) inferred by the parameter estimates of the discrete time GARCH-M model (3.1) at two-daily, daily and two-hourly sampling frequencies. Sample size 40 years. 

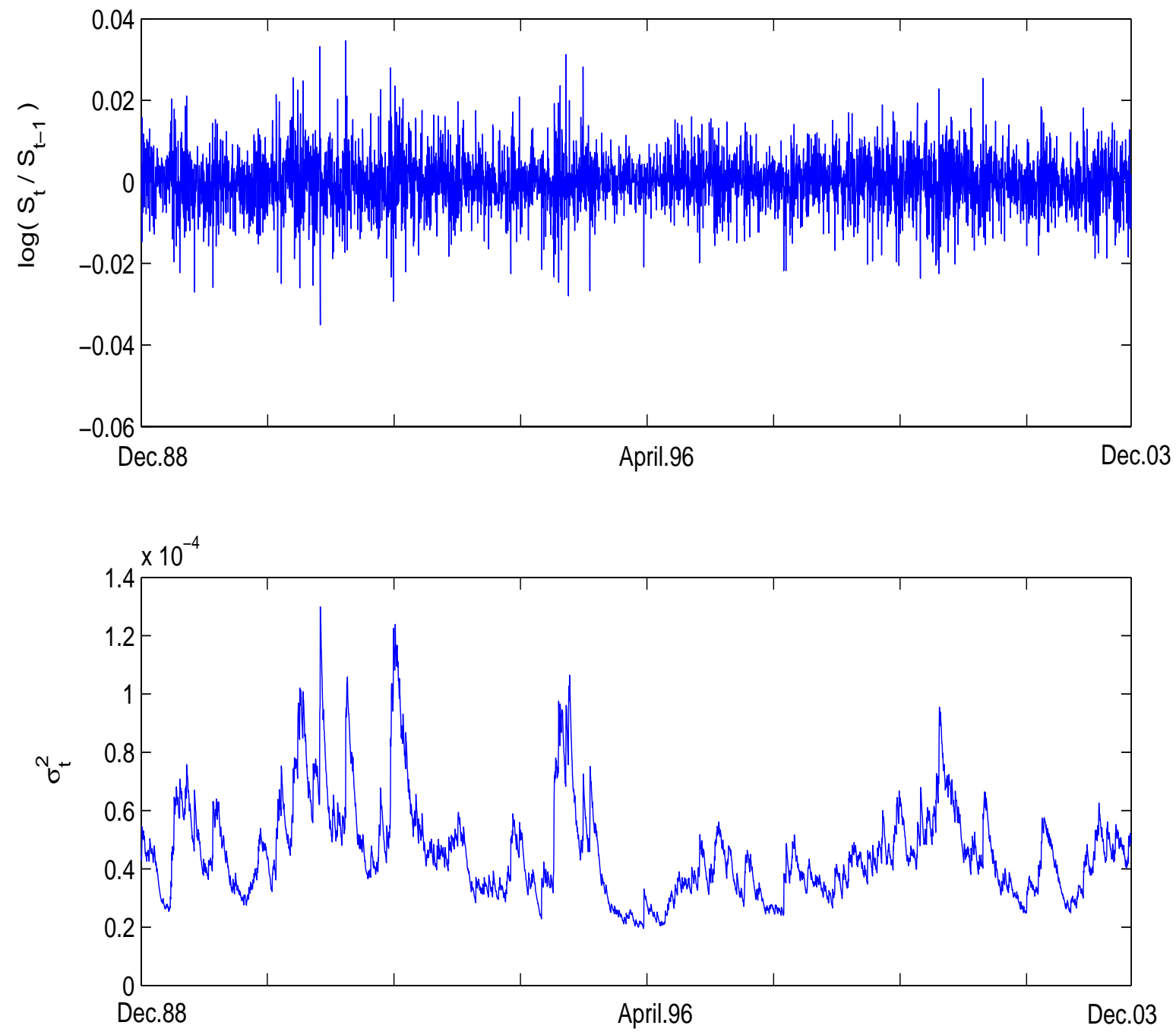

Figure 3.4: DM/US daily exchange rates from December 1988 to December 2003. $\sigma_{t}^{2}$ is defined in equations (3.1). 

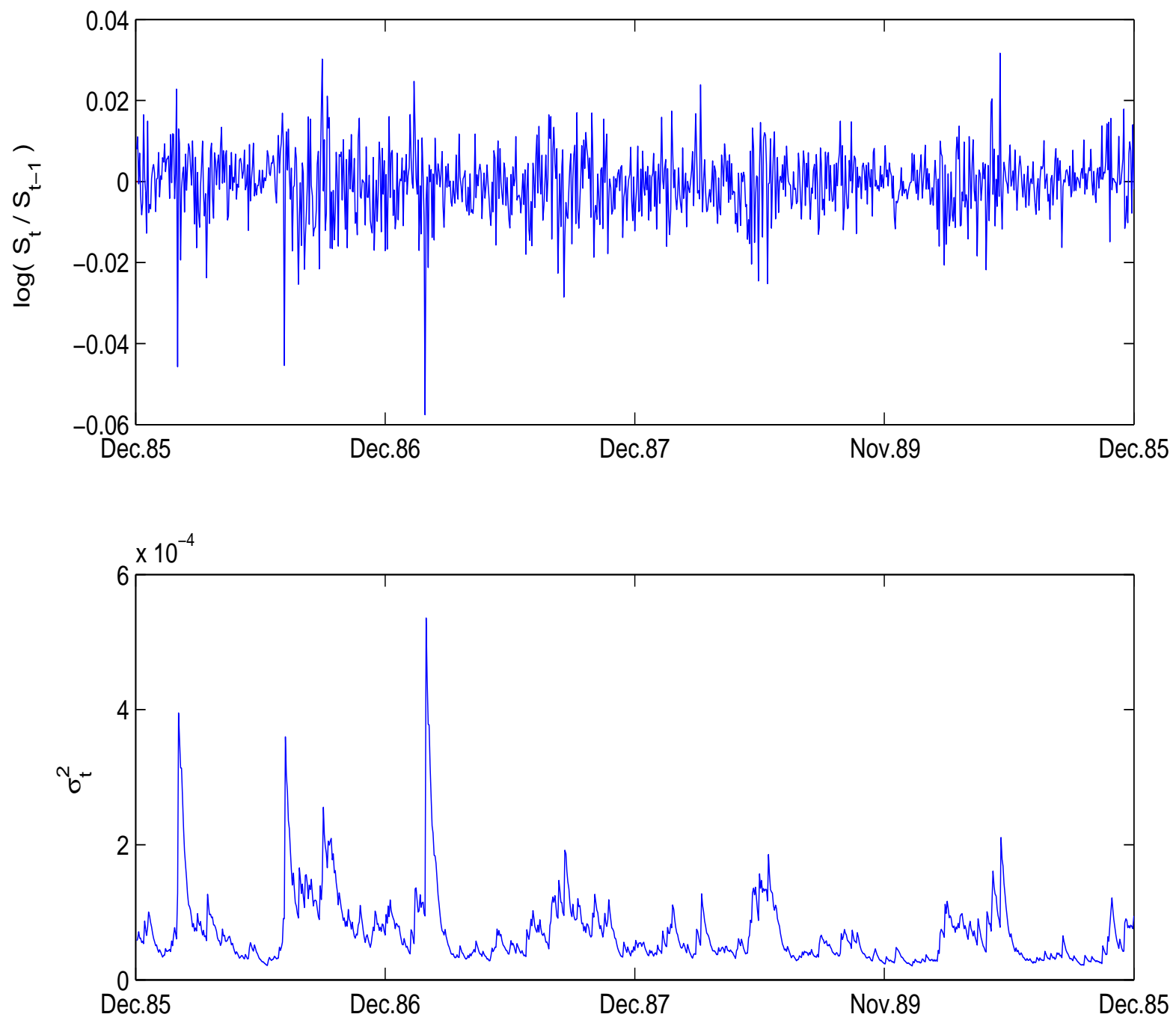

Figure 3.5: DM/US daily exchange rates from January 1985 to October 1988. $\sigma_{t}^{2}$ is defined in equations (3.1). 


\section{Chapter 4}

\section{Conclusions}

In this thesis we derived an analytical closed-form approximation for European option prices when the underlying currency price and its latent variance satisfy a GARCH diffusion model. This model is the continuous-time limit of a standard $\operatorname{GARCH}(1,1)$ in mean model. It generates realistic statistical properties for the underlying currency price. Based on Monte Carlo simulations, we provide evidence for the accuracy of our formula. Simulation studies are made for various sets of parameters, which were derived by earlier empirical studies. Moreover, the properties of implied volatilities induced by the GARCH diffusion process are investigated. The results indicate that the properties of implied volatilities are in agreement with the empirical evidence from currency options literature.

The more specific contributions of this thesis are the following. We derive analytically the first four conditional moments of the integrated variance implied by the GARCH diffusion process. This result has several implications. Firstly and foremost, such conditional moments allow to obtain an analytical closed-form approximation for European option prices under the GARCH diffusion model.

The approximation can be easily implemented in any standard software package. Hence, just plugging in the model parameters, it provides option prices without any computational efforts. Secondly, our analytical approximation allows to easily study volatility surfaces induced by GARCH diffusion models. 
Thirdly, the conditional moments of the integrated variance generalize the conditional moments derived by Hull and White (1987) for log-normal variance processes. Finally, such conditional moments can be used to estimate the continuous time parameters of the GARCH diffusion model using high frequency data; cf. Bollerslev and Zhou (2002).

Nelson's theory suggests an power estimation procedure for the GARCH diffusion model parameters. Using simulated and real data, we find that inference results based on such a procedure are rather accurate. 


\section{Appendix A}

\section{Proof of Propositions and other}

\section{Mathematical Issues}

\section{A.1 Proof of Proposition 2.1}

In the following, we derive the first two conditional moments of the integrated variance $\bar{V}_{T}$ for the GARCH diffusion process,

$$
\bar{V}_{T}=\frac{V_{0}}{T} \int_{0}^{T} d t e^{-\left(c_{2}+\frac{1}{2} c_{3}^{2}\right) t} e^{c_{3} W_{t}}+\frac{c_{1}}{T} \int_{0}^{T} d t \int_{0}^{t} d s e^{\left(c_{2}+\frac{1}{2} c_{3}^{2}\right)(s-t)} e^{c_{3}\left(W_{t}-W_{s}\right)} .
$$

To prove Proposition 2.1 we recall that, if $w$ is a normal random variable $w \sim \mathcal{N}(0, t)$

$$
\mathbb{E}\left[e^{\lambda w}\right]=e^{\frac{\lambda^{2} t}{2}} .
$$

We also need the following lemma

Lemma A.1.1

$\forall x>y>0$,

$$
F(x, y)=e^{-\left(c_{2}+\frac{1}{2} c_{3}^{2}\right)(x+y)} \mathbb{E}\left[e^{c_{3}\left(W_{x}+W_{y}\right)}\right]=e^{-c_{2} x} e^{\left(c_{3}^{2}-c_{2}\right) y} .
$$

$\forall x>y>\alpha>0$,

$$
G(x, y, \alpha)=e^{-\left(c_{2}+\frac{1}{2} c_{3}^{2}\right)(x+y-\alpha)} \mathbb{E}\left[e^{c_{3}\left(W_{x}+W_{y}-W_{\alpha}\right)}\right]=e^{-c_{2} x} e^{\left(c_{3}^{2}-c_{2}\right) y} e^{\left(c_{2}-c_{3}^{2}\right) \alpha} .
$$


$\forall x>\alpha>y>0$,

$$
H(x, y, \alpha)=e^{-\left(c_{2}+\frac{1}{2} c_{3}^{2}\right)(x+y-\alpha)} \mathbb{E}\left[e^{c_{3}\left(W_{x}+W_{y}-W_{\alpha}\right)}\right]=e^{-c_{2}(x+y-\alpha)} .
$$

$\forall x>y>\alpha>\beta>0$,

$$
L(x, y, \alpha, \beta)=e^{-\left(c_{2}+\frac{1}{2} c_{3}^{2}\right)(x+y-\alpha-\beta)} \mathbb{E}\left[e^{c_{3}\left(W_{x}+W_{y}-W_{\alpha}-W_{\beta}\right)}\right]=e^{-c_{2} x} e^{\left(c_{3}^{2}-c_{2}\right) y} e^{\left(c_{2}-c_{3}^{2}\right) \alpha} e^{c_{2} \beta} .
$$

$\forall x>\alpha>y>\beta>0$,

$$
M(x, y, \alpha, \beta)=e^{-\left(c_{2}+\frac{1}{2} c_{3}^{2}\right)(x+y-\alpha-\beta)} \mathbb{E}\left[e^{c_{3}\left(W_{x}+W_{y}-W_{\alpha}-W_{\beta}\right)}\right]=e^{-c_{2}(x+y-\alpha-\beta)} .
$$

Proof. To prove (A.3) we write. $W_{x}+W_{y}=\left(W_{x}-W_{y}\right)+2 W_{y}$. As $\left(W_{x}-W_{y}\right)$ and $W_{y}$ are non-overlapping increments of the Brownian motion $W,\left(W_{x}-W_{y}\right) \sim \mathcal{N}(0, x-y)$ and $2 W_{y} \sim \mathcal{N}(0,4 y)$ one has

$$
\mathbb{E}\left[e^{c_{3}\left(W_{x}+W_{y}\right)}\right]=\mathbb{E}\left[e^{c_{3}\left(W_{x}-W_{y}\right)+2 W_{y}}\right]=\mathbb{E}\left[e^{c_{3}\left(W_{x}-W_{y}\right)}\right] \mathbb{E}\left[e^{c_{3} 2 W_{y}}\right],
$$

then formula (A.3) follows directly from (A.2).

To prove (A.4), use $W_{x}+W_{y}-W_{\alpha}=\left(W_{x}-W_{y}\right)+2\left(W_{y}-W_{\alpha}\right)+W_{\alpha}$.

To prove (A.5), use $W_{x}+W_{y}-W_{\alpha}=\left(W_{x}-W_{\alpha}\right)+W_{y}$.

To prove (A.6), use $W_{x}+W_{y}-W_{\alpha}-W_{\beta}=\left(W_{x}-W_{y}\right)+2\left(W_{y}-W_{\alpha}\right)+W_{\alpha}-W_{\beta}$.

To prove (A.7), use $W_{x}+W_{y}-W_{\alpha}-W_{\beta}=\left(W_{x}-W_{\alpha}\right)+\left(W_{y}-W_{\beta}\right)$.

\section{A.1.1 First conditional moment}

The first conditional moment of $\bar{V}_{T}$ is given by

$$
\begin{aligned}
M_{1}^{g d} & :=\mathbb{E}\left[\bar{V}_{T} \mid V_{0}\right] \\
& =\frac{V_{0}}{T} \int_{0}^{T} d t e^{-\left(c_{2}+\frac{1}{2} c_{3}^{2}\right) t} \mathbb{E}\left[e^{c_{3} W_{t}}\right]+\frac{c_{1}}{T} \int_{0}^{T} d t \int_{0}^{t} d s e^{\left(c_{2}+\frac{1}{2} c_{3}^{2}\right)(s-t)} \mathbb{E}\left[e^{c_{3}\left(W_{t}-W_{s}\right)}\right] .
\end{aligned}
$$


As $W_{t} \sim \mathcal{N}(0, t)$ and $W_{t}-W_{s} \sim \mathcal{N}(0, t-s)$, using (A.2) we get the first conditional moment (2.8) in Proposition 2.1,

$$
\begin{aligned}
M_{1}^{g d} & :=\frac{V_{0}}{T} \int_{0}^{T} d t e^{-\left(c_{2}+\frac{1}{2} c_{3}^{2}\right) t} e^{\frac{1}{2} c_{3}^{2} t}+\frac{c_{1}}{T} \int_{0}^{T} d t \int_{0}^{t} d s e^{\left(c_{2}+\frac{1}{2} c_{3}^{2}\right)(s-t)} e^{\frac{1}{2} c_{3}^{2}(t-s)} \\
& =V_{0} \int_{0}^{T} e^{-c_{2} t} d t+c_{1} \int_{0}^{T} d t \int_{0}^{t} d s e^{c_{2}(s-t)}=\frac{V_{0}}{c_{2}}\left(\frac{1-e^{-c_{2} T}}{T}\right)+\frac{c_{1}}{T} \int_{0}^{T} \frac{1-e^{-c_{2} t}}{c_{2}} d t \\
& =\frac{c_{1}}{c_{2}}+\left(V_{0}-\frac{c_{1}}{c_{2}}\right) \frac{1-e^{-c_{2} T}}{c_{2} T} .
\end{aligned}
$$

\section{A.1.2 Second conditional moment}

The second conditional moment of $\bar{V}_{T}$ is given by

$$
\begin{aligned}
& \mathbb{E}\left[\bar{V}_{T}^{2} \mid V_{0}\right]=\mathbb{E}\left[\frac{1}{T^{2}} \int_{0}^{T} d r_{2} \int_{0}^{T} d r_{1}\left(V_{r_{1}} V_{r_{2}}\right)\right]=\frac{1}{T^{2}} \int_{0}^{T} d r_{2} \int_{0}^{T} d r_{1} \mathbb{E}\left[V_{r_{1}} V_{r_{2}}\right] \\
& =\frac{2 !}{T^{2}} \int_{0}^{T} d r_{2} \int_{0}^{r_{2}} d r_{1} \mathbb{E}\left[V_{r_{1}} V_{r_{2}}\right] \\
& =\frac{2 !}{T^{2}} \int_{0}^{T} d r_{2} \int_{0}^{r_{2}} d r_{1}(\mathbb{E}[A]+\mathbb{E}[B]+\mathbb{E}[C]+\mathbb{E}[D]),
\end{aligned}
$$

where

$$
\begin{aligned}
& A:=V_{0}^{2} e^{-\left(c_{2}+\frac{1}{2} c_{3}^{2}\right)\left(r_{1}+r_{2}\right)+c_{3}\left(W_{r_{1}}+W_{r_{2}}\right)}, \\
& B:=c_{1} V_{0} e^{-\left(c_{2}+\frac{1}{2} c_{3}^{2}\right) r_{1}+c_{3} W_{r_{1}}} \int_{0}^{r_{2}} d s_{2} e^{\left(c_{2}+\frac{1}{2} c_{3}^{2}\right)\left(s_{2}-r_{2}\right)+c_{3}\left(W_{r_{2}}-W_{s_{2}}\right)}, \\
& C:=c_{1} V_{0} e^{-\left(c_{2}+\frac{1}{2} c_{3}^{2}\right) r_{2}+c_{3} W_{r_{2}}} \int_{0}^{r_{1}} d s_{1} e^{\left(c_{2}+\frac{1}{2} c_{3}^{2}\right)\left(s_{1}-r_{1}\right)+c_{3}\left(W_{r_{1}}-W_{s_{1}}\right)}, \\
& D:=c_{1}^{2} \int_{0}^{r_{1}} d s_{2} \int_{0}^{r_{2}} d s_{1} e^{\left(c_{2}+\frac{1}{2} c_{3}^{2}\right)\left(s_{1}-r_{1}+s_{2}-r_{2}\right)} e^{c_{3}\left(W_{r_{1}}-W_{s_{1}}+W_{r_{2}}-W_{s_{2}}\right)} .
\end{aligned}
$$

We compute each addend in (A.8).

- Calculation of

$$
\begin{aligned}
& \frac{2}{T^{2}} \int_{0}^{T} d r_{2} \int_{0}^{r_{2}} d r_{1} \mathbb{E}[A]= \\
& \quad \frac{2}{T^{2}} \int_{0}^{T} d r_{2} \int_{0}^{r_{2}} d r_{1} V_{0}^{2} e^{-\left(c_{2}+\frac{1}{2} c_{3}^{2}\right)\left(r_{1}+r_{2}\right)} \mathbb{E}\left[e^{c_{3}\left(W_{r_{1}}+W_{r_{2}}\right)}\right] .
\end{aligned}
$$


As $r_{2}>r_{1}>0$, we use formula (A.3) with $x=r_{2}$ and $y=r_{1}$

$$
\frac{2}{T^{2}} \int_{0}^{T} d r_{2} \int_{0}^{r_{2}} d r_{1} \mathbb{E}[A]=\frac{2 V_{0}^{2}}{T^{2}} \int_{0}^{T} d r_{2} \int_{0}^{r_{2}} d r_{1} F\left(r_{2}, r_{1}\right)
$$

and iterating integrations

$$
\frac{2}{T^{2}} \int_{0}^{T} d r_{2} \int_{0}^{r_{2}} d r_{1} \mathbb{E}[A]=\frac{2 V_{0}^{2}}{T^{2}}\left[\frac{e^{-\left(2 c_{2}-c_{3}^{2}\right) T}}{\left(c_{3}^{2}-2 c_{2}\right)\left(c_{3}^{2}-c_{2}\right)}+\frac{e^{-c_{2} T}}{c_{2}\left(c_{3}^{2}-c_{2}\right)}-\frac{1}{c_{2}\left(c_{3}^{2}-2 c_{2}\right)}\right]
$$

- Calculation of

$$
\begin{aligned}
& \frac{2}{T^{2}} \int_{0}^{T} d r_{2} \int_{0}^{r_{2}} d r_{1} \mathbb{E}[B]= \\
& \quad \frac{2 c_{1} V_{0}}{T^{2}} \int_{0}^{T} d r_{2} \int_{0}^{r_{2}} d r_{1} \int_{0}^{r_{1}} d s_{1} e^{-\left(c_{2}+\frac{1}{2} c_{3}^{2}\right)\left(r_{2}+r_{1}-s_{1}\right)} \mathbb{E}\left[e^{c_{3}\left(W_{r_{2}}+W_{r_{1}}-W_{s_{1}}\right)}\right]
\end{aligned}
$$

As $r_{2}>r_{1}>s_{1}>0$, we use formula (A.4) with $x=r_{2}, y=r_{1}$ and $\alpha=s_{1}$ to get

$$
\begin{aligned}
& \frac{2}{T^{2}} \int_{0}^{T} d r_{2} \int_{0}^{r_{2}} d r_{1} \mathbb{E}[B]=\frac{2 c_{1} V_{0}}{T^{2}} \int_{0}^{T} d r_{2} \int_{0}^{r_{2}} d r_{1} \int_{0}^{r_{1}} d s_{1} G\left(r_{2}, r_{1}, s_{1}\right) \\
& \quad=\frac{c_{1} V_{0}}{T^{2} c_{2}^{4}\left(c_{2}-c_{3}^{2}\right)^{2}\left(-2 c_{2}+c_{3}^{2}\right)} \times \\
& \quad\left[-c_{2} e^{-T c_{2}}\left(-2 c_{2}+c_{3}^{2}\right)\left(c_{2}^{2}\left(-2+T c_{2}\right)+2 c_{2} c_{3}^{2}-\left(2+T c_{2}\right) c_{3}^{4}\right)\right. \\
& \left.\quad+c_{2}\left(c_{2}-c_{3}^{2}\right)^{2}\left(-2 c_{2}\left(-1+T c_{2}\right)+\left(-2+T c_{2}\right) c_{3}^{2}\right)+2 c_{2}^{4} e^{T\left(c_{3}^{2}-2 c_{2}\right)}\right] .
\end{aligned}
$$

- Calculation of

$$
\frac{2}{T^{2}} \int_{0}^{T} d r_{2} \int_{0}^{r_{2}} d r_{1} \mathbb{E}[C]
$$

Simply notice that

$$
\int_{0}^{T} d r_{2} \int_{0}^{T} d r_{1} \mathbb{E}[B]=\int_{0}^{T} d r_{2} \int_{0}^{T} d r_{1} \mathbb{E}[C]
$$


- Calculation of

$$
\begin{aligned}
& \frac{2}{T^{2}} \int_{0}^{T} d r_{2} \int_{0}^{r_{2}} d r_{1} \mathbb{E}[D]= \\
& \quad \frac{2 c_{1}^{2}}{T^{2}} \int_{0}^{T} d r_{2} \int_{0}^{r_{2}} d r_{1} \int_{0}^{r_{2}} d s_{2} \int_{0}^{r_{1}} d s_{1}\left(e^{-\left(c_{2}+\frac{1}{2} c_{3}^{2}\right)\left(r_{2}+r_{1}-s_{2}-s_{1}\right)} \mathbb{E}\left[e^{c_{3}\left(W_{r_{2}}+W_{r_{1}}-W_{s_{1}}-W_{s_{2}}\right)}\right]\right) .
\end{aligned}
$$

We divide the integration domain of $s_{2}$ and $s_{1}$ as follows

$$
\begin{aligned}
& \frac{2}{T^{2}} \int_{0}^{T} d r_{2} \int_{0}^{r_{2}} d r_{1} \mathbb{E}[D]= \\
& \quad \frac{2 c_{1}^{2}}{T^{2}} \int_{0}^{T} d r_{2} \int_{0}^{r_{2}} d r_{1} \int_{0}^{r_{1}} d s_{2} \int_{0}^{s_{2}} d s_{1}(\ldots)+ \\
& \quad+\frac{2 c_{1}^{2}}{T^{2}} \int_{0}^{T} d r_{2} \int_{0}^{r_{2}} d r_{1} \int_{0}^{r_{1}} d s_{2} \int_{s_{2}}^{r_{1}} d s_{1}(\ldots)+ \\
& \quad+\frac{2 c_{1}^{2}}{T^{2}} \int_{0}^{T} d r_{2} \int_{0}^{r_{2}} d r_{1} \int_{r_{1}}^{r_{2}} d s_{2} \int_{0}^{r_{1}} d s_{1}(\ldots) .
\end{aligned}
$$

The previous partition allows us to use

formula (A.6) with $x=r_{2}, y=r_{1}, \alpha=s_{2}, \beta=s_{1}$ in (A.12) as $T>r_{2}>r_{1}>s_{2}>s_{1}>0$;

formula (A.6) with $x=r_{2}, y=r_{1}, \alpha=s_{1}, \beta=s_{2}$ in (A.13) as $T>r_{2}>r_{1}>s_{1}>s_{2}>0$;

formula (A.7) with $x=r_{2}, y=r_{1}, \alpha=s_{2}, \beta=s_{1}$ in (A.14) as $T>r_{2}>s_{2}>r_{1}>s_{1}>0$; then

$$
\begin{aligned}
& \frac{2}{T^{2}} \int_{0}^{T} d r_{2} \int_{0}^{r_{2}} d r_{1} \mathbb{E}[D]= \\
& \quad=\frac{2 c_{1}^{2}}{T^{2}} \int_{0}^{T} d r_{2} \int_{0}^{r_{2}} d r_{1} \int_{0}^{r_{1}} d s_{2} \int_{0}^{s_{2}} d s_{1} L\left(r_{2}, r_{1}, s_{2}, s_{1}\right) \\
& \quad+\frac{2 c_{1}^{2}}{T^{2}} \int_{0}^{T} d r_{2} \int_{0}^{r_{2}} d r_{1} \int_{0}^{r_{1}} d s_{2} \int_{s_{2}}^{r_{1}} d s_{1} L\left(r_{2}, r_{1}, s_{1}, s_{2}\right) \\
& \quad+\frac{2 c_{1}^{2}}{T^{2}} \int_{0}^{T} d r_{2} \int_{0}^{r_{2}} d r_{1} \int_{r_{1}}^{r_{2}} d s_{2} \int_{0}^{r_{1}} d s_{1} M\left(r_{2}, r_{1}, s_{2}, s_{1}\right)
\end{aligned}
$$


and iterating integrations

$$
\begin{aligned}
& \frac{2}{T^{2}} \int_{0}^{T} \int_{0}^{r_{2}} \mathbb{E}[D] d r_{1} d r_{2}=\frac{c_{1}^{2}}{T^{2} c_{2}^{4}\left(c_{2}-c_{3}^{2}\right)^{2}\left(-2 c_{2}+c_{3}^{2}\right)^{2}} \times \\
& \quad\left[-2 e^{-T c_{2}}\left(-2 c_{2}+c_{3}^{2}\right)^{2}\left(c_{2}^{2}-T c_{2}^{3}-2 c_{2} c_{3}^{2}+\left(3+T c_{2}\right) c_{3}^{4}\right)\right. \\
& \quad+\left(c_{2}-c_{3}^{2}\right)^{2}\left(\left(4 c_{2}^{2}\left(-1+T c_{2}\right)^{2}-4 c_{2}\left(4+T c_{2}\left(-3+T c_{2}\right)\right) c_{3}^{2}+\left(6+T c_{2}\left(-4+T c_{2}\right)\right) c_{3}^{4}\right)+\right. \\
& \left.\quad+4 e^{T\left(c_{3}^{2}-2 c_{2}\right)} c_{2}^{4}\right] .
\end{aligned}
$$

Summing (A.9), (A.10), (A.11) and (A.15) we get the second conditional moment of $\bar{V}_{T}$ :

$$
\begin{aligned}
M_{2}^{g d}:=\mathbb{E}\left[\bar{V}_{T}^{2} \mid V_{0}\right]= & \frac{1}{T^{2} c_{2}^{4}\left(c_{2}-c_{3}^{2}\right)^{2}\left(-2 c_{2}+c_{3}^{2}\right)^{2}} \\
& {\left[e ^ { - 2 T c _ { 2 } } \left(-2 e^{T c_{2}}\left(-2 c_{2}+c_{3}^{2}\right)^{2}\right.\right.} \\
& \left(c_{1}^{2}\left(c_{2}^{2}-T c_{2}^{3}-2 c_{2} c_{3}^{2}+\left(3+T c_{2}\right) c_{3}^{4}\right)\right. \\
& +c_{1} c_{2}\left(c_{2}^{2}\left(-2+T c_{2}\right)+2 c_{2} c_{3}^{2}-\left(2+T c_{2}\right) c_{3}^{4}\right) V_{0}+ \\
& \left.c_{2}^{3}\left(c_{2}-c_{3}^{2}\right) V_{0}^{2}\right)+e^{2 T c_{2}}\left(c_{2}-c_{3}^{2}\right)^{2}\left(c _ { 1 } ^ { 2 } \left(4 c_{2}^{2}\left(-1+T c_{2}\right)^{2}\right.\right. \\
& \left.-4 c_{2}\left(4+T c_{2}\left(-3+T c_{2}\right)\right) c_{3}^{2}+\left(6+T c_{2}\left(-4+T c_{2}\right)\right) c_{3}^{4}\right)+ \\
& 2 c_{1} c_{2}\left(2 c_{2}-c_{3}^{2}\right)\left(2 c_{2}\left(-1+T c_{2}\right)-\left(-2+T c_{2}\right) c_{3}^{2}\right) \\
& \left.V_{0}+2 c_{2}^{3}\left(2 c_{2}-c_{3}^{2}\right) V_{0}^{2}\right)+2 e^{T} c_{3}^{2} c_{2}^{4}\left(2 c_{1}^{2}-2 c_{1}\left(2 c_{2}-c_{3}^{2}\right) V_{0}+\right. \\
& \left.\left.\left.\left(2 c_{2}^{2}-3 c_{2} c_{3}^{2}+c_{3}^{4}\right) V_{0}^{2}\right)\right)\right]
\end{aligned}
$$

The second central conditional moment of $\bar{V}_{T}, M_{2 c}^{g d}$, stated in (2.9) Proposition 2.1 is given by $M_{2 c}^{g d}=$ $M_{2}^{g d}-\left(M_{1}^{g d}\right)^{2}$.

\section{A.2 Conditional Moments of $\bar{V}_{T}$ under Log-Normal Variance}

The Hull and White approximation formula for European option prices, based on equation (2.6), involves the conditional moments of the integrated variance $\bar{V}_{T}$. In the following, we show that such approximation formula does not hold for long maturity options when the variance of the asset price is 
driven by a log-normal process

$$
d V_{t}=c_{2} V_{t} d t+c_{3} V_{t} d W_{t}
$$

such as in the Hull and White (1987) option pricing model.

We consider the case $c_{2}=0$ (log-normal process without drift) and the case $c_{2} \neq 0$ (log-normal process with drift).

1. Case $c_{2}=0$ and $c_{3} \neq 0$. Hull and White show that

$$
\begin{aligned}
& M_{1}^{l n}:=\mathbb{E}\left[\bar{V}_{T} \mid V_{0}\right]=V_{0}, \\
& M_{2 c}^{l n}:=\mathbb{E}\left[\left(\bar{V}_{T}-M_{1}^{l n}\right)^{2} \mid V_{0}\right]=2 V_{0}^{2}\left[\frac{e^{c_{3}^{2} T}-c_{3}^{2} T-1}{c_{3}^{4} T^{2}}-\frac{1}{2}\right] .
\end{aligned}
$$

When $T \longrightarrow \infty, M_{2 c}^{l n} \longrightarrow \infty$ and

$$
M_{2 c}^{l n} \approx 2 V_{0}^{2}\left[\frac{e^{c_{3}^{2} T}}{c_{3}^{4} T^{2}}\right] \text { for } c_{3}^{2} T \gg 1 .
$$

Hence, in the case $c_{3}^{2} T \gg 1$, the Hull and White approximation series does not hold.

2. Case $c_{2} \neq 0$ and $c_{3} \in \mathbb{R}^{+} \backslash\left\{c_{2},-2 c_{2}\right\}$. Hull and White show that

$$
\begin{aligned}
M_{1 c}^{l n}=V_{0} & {\left[\frac{e^{c_{2} T}-1}{c_{2} T}\right], } \\
M_{2 c}^{l n}=V_{0}^{2} & {\left[\frac{2 e^{\left(2 c_{2}+c_{3}^{2}\right) T}}{\left(c_{3}^{2}+c_{2}\right)\left(c_{3}^{2}+2 c_{2}\right) T^{2}}-\frac{e^{2 c_{2} T}}{c_{2}^{2} T^{2}}\right.} \\
& \left.+\frac{e^{c_{2} T}}{c_{2}^{2} T^{2}}\left(\frac{2 c_{3}^{2}}{c_{3}^{2}+c_{2}}\right)-\frac{1}{c_{2}^{2} T^{2}}\left(\frac{c_{3}^{2}}{c_{3}^{2}+2 c_{2}}\right)\right] .
\end{aligned}
$$

When $c_{2}<0$ and $T \longrightarrow \infty$,

$$
M_{1 c}^{l n} \approx \frac{V_{0}}{-c_{2} T} \longrightarrow 0
$$

and if $c_{3}^{2}<-2 c_{2}$,

$$
M_{2 c}^{l n} \approx \frac{-c_{3}^{2}}{c_{2}^{2} T^{2}\left(c_{3}^{2}+2 c_{2}\right)} \longrightarrow 0
$$


otherwise if $c_{3}^{2}>-2 c_{2}$,

$$
M_{2 c}^{l n} \approx \frac{2 e^{\left(2 c_{2}+c_{3}^{2}\right) T}}{\left(c_{3}^{2}+c_{2}\right)\left(c_{3}^{2}+2 c_{2}\right) T^{2}} \longrightarrow \infty .
$$

When $c_{2}>0$ and $T \longrightarrow \infty$,

$$
M_{1 c}^{l n} \approx V_{0}, \text { and } M_{2 c}^{l n} \approx \frac{2 e^{\left(2 c_{2}+c_{3}^{2}\right) T}}{\left(c_{3}^{2}+c_{2}\right)\left(c_{3}^{2}+2 c_{2}\right) T^{2}} \longrightarrow \infty
$$

Hence, when the time to maturities increases, the series formula based on equation (2.6) degenerates.

A log-normal variance process can be adopted only for short maturity options. Moreover, the solution of the equation (A.17) is

$$
V_{t}=V_{0} e^{\left(c_{2}-c_{3}^{2} / 2\right) t+c_{3} W_{t}},
$$

and for the law of iterated logarithm and the law of large numbers (cf. Karatzas and Shreve 1988),

1. if $c_{2}<c_{3}^{2} / 2$,

$$
\lim _{t \longrightarrow \infty} V_{t}=0, \quad \text { a.s. }
$$

2. if $c_{2}>c_{3}^{2} / 2$,

$$
\lim _{t \longrightarrow \infty} V_{t}=\infty, \quad \text { a.s. }
$$

3. if $c_{2}=c_{3}^{2} / 2$,

$$
\begin{aligned}
& \inf _{0 \leq t \leq \infty} V_{t}=0, \quad \text { a.s. } \\
& \sup _{0 \leq t \leq \infty} V_{t}=\infty, \quad \text { a.s. }
\end{aligned}
$$

Hence, when $t \longrightarrow \infty, V_{t} \longrightarrow 0$ or $V_{t} \longrightarrow \infty$, and this is in contrast with the observed mean reversion of implied and historical volatilities. 


\section{A.3 The Market Price of Risk and the Novikov's Condition}

In this appendix, using the Novikov's condition we show that the change of measure from $\mathbb{P}$ to $\mathbb{P}^{*}$ in Section 2.1 is well defined. Recall that $\mathbb{P}^{*}$ is a martingale measure, i.e. a probability measure, equivalent to the objective probability measure $\mathbb{P}$, under which discounted asset price processes are $\mathcal{F}_{t}$-adapted martingales. Such a measure ensures the absence of arbitrage opportunities and is unique if and only if the market is complete; cf. Harrison and Kreps (1979), Harrison and Pliska (1981) and Delbaen and Schachermayer (1994). In stochastic volatility setting markets are incomplete. Hence, the martingale measure is not unique and any such a measure implies admissible arbitrage free option prices.

The data generating process is given by equations (1.7)-(1.8)

$$
d S_{t}=\mu S_{t} d t+\sqrt{V_{t}} S_{t} d B_{t}, \quad d V_{t}=\left(c_{1}-c_{2} V_{t}\right) d t+c_{3} V_{t} d W_{t}
$$

where $\left(B_{t}, W_{t}\right)$ is a two-dimensional Brownian motion on $\left(\Omega, \mathcal{F}, \mathcal{F}_{t}, \mathbb{P}\right)$.

From the integral form of martingale representation (cf. Karatzas and Shreve (1991), any equivalent martingale measure $\mathbb{P}^{*}$ is characterized by the density process with respect to $\mathbb{P}$. This density can be written as

$$
M_{t}\left(\lambda_{t}^{(1)}, \lambda_{t}^{(2)}\right):=\left.\frac{d \mathbb{P}^{*}}{d \mathbb{P}}\right|_{\mathcal{F}_{t}}=\exp \left(\int_{0}^{t} \lambda_{s}^{(1)} d B_{s}-\frac{1}{2} \int_{0}^{t} \lambda_{s}^{(1)^{2}} d s+\int_{0}^{t} \lambda_{s}^{(2)} d W_{s}-\frac{1}{2} \int_{0}^{t} \lambda_{s}^{(2)^{2}} d s\right)
$$

where $\left(\lambda^{(1)}, \lambda^{(2)}\right)$ is an $\mathcal{F}_{t}$-adapted process and satisfies the integrability conditions $\int_{0}^{T} \lambda_{s}^{(1)^{2}} d s<\infty$ and $\int_{0}^{T} \lambda_{s}^{(2)^{2}} d s<\infty, a . s$. By the Girsanov's theorem, the process $\left(B_{t}^{*}, W_{t}^{*}\right)$ defined by

$$
B_{t}^{*}=B_{t}+\int_{0}^{t} \lambda_{s}^{(1)} d s \text { and } W_{t}^{*}=W_{t}+\int_{0}^{t} \lambda_{s}^{(2)} d s
$$

is a two-dimensional Brownian motion under $\mathbb{P}^{*}$. Hence, (1.7)-(1.8) and (A.23) imply the dynamic of the asset price under $\mathbb{P}^{*}$

$$
d S_{t}=\left(\mu-\sqrt{V_{t}} \lambda_{t}^{(1)}\right) S_{t} d t+\sqrt{V_{t}} S_{t} d B_{t}^{*},
$$




$$
d V_{t}=\left(c_{1}-c_{2} V_{t}-c_{3} V_{t} \lambda_{t}^{(2)}\right) d t+c_{3} V_{t} d W_{t}^{*}
$$

By Itô's lemma, the stochastic differential equation driving the discounted process $S_{t}^{(d)}:=e^{-r t} S_{t}$ under $\mathbb{P}^{*}$ is

$$
d S_{t}^{(d)}=\left(\mu-r-\sqrt{V_{t}} \lambda_{t}^{(1)}\right) S_{t}^{(d)} d t+\sqrt{V_{t}} S_{t}^{(d)} d B_{t}^{*},
$$

where $r:=r_{d}-r_{f}$. To ensure no arbitrage opportunity in the economy, $S^{(d)}$ has to be a $\mathbb{P}^{*}$-martingale then the stochastic differential equation (A.24) has to be driftless

$$
\mu-r=\sqrt{V_{t}} \lambda_{t}^{(1)}, \mathbb{P}^{*}-a . s .
$$

The process $\left(\lambda^{(1)}, \lambda^{(2)}\right)$ is interpreted as the risk premia associated to the two sources of uncertainty in the economy, $B$ and $W$. As $S$ is the only traded asset, the no arbitrage condition (A.25) determines only $\lambda_{t}^{(1)}$. Any 'proper' choice of the volatility risk premium process $\lambda_{t}^{(2)}, 0 \leq t \leq T$, gives a martingale measure $\mathbb{P}^{*}$ characterized by the density process $M_{t}\left((\mu-r) V_{t}^{-1 / 2}, \lambda_{t}^{(2)}\right)$ with respect to $\mathbb{P}$. Hence, $\mathbb{P}^{*}$ is not unique; cf. Harrison and Pliska $(1981,1983)$.

We assume

$$
\lambda_{t}^{(2)}=\left(c_{2}^{*}-c_{2}\right) / c_{3}, \forall t,
$$

which implies the risk neutral dynamic of $V$ in $(2.3)$

$$
d V_{t}=\left(c_{1}-c_{2}^{*} V_{t}\right) d t+c_{3} V_{t} d W_{t}^{*}
$$

Given the risk premia (A.25)-(A.26), the Radon Nikodym derivative is

$$
M_{t}:=\left.\frac{d \mathbb{P}^{*}}{d \mathbb{P}}\right|_{\mathcal{F}_{t}}=\exp \left(\int_{0}^{t} \frac{\mu-r}{\sqrt{V_{s}}} d B_{s}-\frac{1}{2} \int_{0}^{t} \frac{(\mu-r)^{2}}{V_{s}} d s+\frac{c_{2}^{*}-c_{2}}{c_{3}} W_{t}-\frac{1}{2} \frac{\left(c_{2}^{*}-c_{2}\right)^{2}}{c_{3}^{2}} t\right)
$$

is 'well-defined' when the (sufficient) Novikov's condition is satisfied,

$$
\mathbb{E}_{\mathbb{P}}\left[\exp \int_{0}^{T} \frac{\lambda_{s}^{(1)^{2}}+\lambda_{s}^{(2)^{2}}}{2} d s\right]=\exp \left(\frac{\left(c_{2}^{*}-c_{2}\right)^{2} T}{2 c_{3}^{2}}\right) \mathbb{E}_{\mathbb{P}}\left[\exp \int_{0}^{T} \frac{(\mu-r)^{2}}{2 V_{s}} d s\right]<\infty .
$$


Jones (2003) provide an upper bound for the expectation in (A.28) using Jensen's inequality and Fubini's theorem. Since the exponential is a convex function,

$$
\mathbb{E}_{\mathbb{P}}\left[\exp \left(\frac{1}{2} \int_{0}^{T} \frac{(\mu-r)^{2}}{V_{s}} d s\right)\right] \leq \int_{0}^{T} \mathbb{E}_{\mathbb{P}}\left[\exp \frac{(\mu-r)^{2}}{2 V_{s}}\right] d s,
$$

and we have to show the finiteness of $\mathbb{E}\left[\exp \left(\frac{1}{2}(\mu-r)^{2} V_{s}^{-1}\right)\right]$. As the stationary distribution of $V$ is the Inverse Gamma distribution with parameters $r=1+2 c_{2} / c_{3}^{2}$ and $s=2 c_{1} / c_{3}^{2}$,

$$
\mathbb{E}_{\mathbb{P}}\left[\exp \left(\frac{(\mu-r)^{2}}{2 V_{s}}\right)\right]=\int_{0}^{+\infty} \exp \left(\frac{1}{2}\left((\mu-r)^{2}-s\right) g\right) \frac{s^{r}}{\Gamma(r)} g^{r-1} d g<\infty .
$$

This expectation is finite when

$$
(\mu-r)^{2}<2 s
$$

otherwise the integral in (A.30) does not converge on $(0, \infty)$. For most reasonable continuous time parameters of the GARCH diffusion model $(\mu-r)^{2}$ is smaller than 0.04 and $2 s$ is always greater than 0.2 , then condition (A.31) should be satisfied.

\section{A.4 Nelson's Moment Matching Procedure}

In this appendix, using the moment matching procedure in Nelson (1990) we characterize the discrete time GARCH(1,1)-M sequence which converges to the continuous time GARCH diffusion model.

Recall, the sequence of discrete time $\operatorname{GARCH}(1,1)-\mathrm{M}$ model in (3.1), depending on $h$, is

$$
\begin{aligned}
Y_{k h} & =Y_{(k-1) h}+\left[\mu-\frac{\sigma_{k h}^{2}}{2}\right] h+\sigma_{k h} \sqrt{h} Z_{k h}, \\
\sigma_{(k+1) h}^{2} & =w^{(h)}+\beta^{(h)} \sigma_{k h}^{2}+\alpha^{(h)} \sigma_{k h}^{2} Z_{k h}^{2},
\end{aligned}
$$

where $Y_{k h}:=\log \left(S_{k h}\right), k \in \mathbb{N}, Z_{k h} \sim$ i.i.d.N $\mathcal{N}(0,1)$ and $\sigma_{k h}^{2}$ is the conditional variance of $\left(Y_{k h}-Y_{(k-1) h}\right)$ given the $\sigma$-algebra $M_{k h}$ generated by $\left\{Y_{0}, \ldots, Y_{(k-1) h}\right\}$ and $\left\{\sigma_{0}^{2}, \ldots, \sigma_{k h}^{2}\right\}$. Assume that

$$
\mathbb{P}\left[\left(Y_{0}, \sigma_{0}^{2}\right) \in \Gamma\right]=\nu_{h}(\Gamma), \quad \forall \Gamma \in \mathcal{B}\left(\mathbb{R}^{2}\right),
$$


such that $\left\{\nu_{h}\right\}_{h \downarrow 0}=\nu_{0}$, where $\nu_{0}$ is the probability measure of the GARCH diffusion model $\left(\log S_{t}, V_{t}\right)$ at time $t=0$, and assume that for each $h \geq 0, \nu_{h}\left(\left(Y_{0}, \sigma_{0}^{2}\right): \sigma_{0}^{2}>0\right)=1$. Define the sequence of continuous time process $\left(Y_{t}, \sigma_{t}^{2}\right)$ as step functions from the sequence of the discrete time $\operatorname{GARCH}(1,1)$ M model in (3.1)

$$
Y_{t}=Y_{k h} \text { and } \sigma_{t}^{2}=\sigma_{k h}^{2}, \text { for } k h \leq t<(k+1) h
$$

where $\sigma_{t}^{2}$ represents the variance per unit of time $h$. Nelson show that when the drift and the second moment of $\left(Y_{k h}, \sigma_{(k+1) h}^{2}\right)$ per unit of time converge to well-behaved limits and the first difference of $\left(Y_{k h}, \sigma_{(k+1) h}^{2}\right)$ have the fourth moment that converges to zero at a proper rate when $h$ goes to zero, $\left(Y_{t}, \sigma_{t}^{2}\right)$ converges in distribution to the GARCH diffusion model (1.13).

In the following, we look for the sequence $\left\{w^{(h)}, \beta^{(h)}, \alpha^{(h)}\right\} \in\left(\mathbb{R}^{+}\right)^{3}$ which implies the previous convergence ${ }^{1}$.

The discrete time process (3.1) is Markovian and the drift per unit of time of $\left(Y_{k h}, \sigma_{(k+1) h}^{2}\right)$ is

$$
\begin{aligned}
\mathbb{E}\left[\frac{Y_{k h}-Y_{(k-1) h}}{h} \mid M_{k h}\right] & =\mu-\frac{\sigma_{k h}^{2}}{2}, \\
\mathbb{E}\left[\frac{\sigma_{(k+1) h}^{2}-\sigma_{k h}^{2}}{h} \mid M_{k h}\right] & =\frac{w^{(h)}}{h}+\sigma_{k h}^{2} \frac{\left(\beta^{(h)}+\alpha^{(h)} \mathbb{E}\left[Z_{k h}^{2} \mid M_{k h}\right]-1\right)}{h} \\
& =\frac{w^{(h)}}{h}+\sigma_{k h}^{2} \frac{\left(\beta^{(h)}+\alpha^{(h)}-1\right)}{h} .
\end{aligned}
$$

The drift per unit of time has to converge to the drift per unit of time of the GARCH diffusion model, $\left(c_{1}-c_{2} V\right)$, and this implies

$$
\begin{aligned}
& \lim _{h \downarrow 0} \frac{w^{(h)}}{h}=c_{1}, \\
& \lim _{h \downarrow 0} \frac{\left(\beta^{(h)}+\alpha^{(h)}-1\right)}{h}=c_{2} .
\end{aligned}
$$

Equations (A.36)-(A.37) give the relation between $\beta^{(h)}, \alpha^{(h)}, w^{(h)}$ and $c_{1}, c_{2}$.

\footnotetext{
${ }^{1}$ Notice that $\left\{w^{(h)}, \beta^{(h)}, \alpha^{(h)}\right\} \in\left(\mathbb{R}^{+}\right)^{3}$ ensures a positive $\sigma_{t}^{2}$ process.
} 
The second moments per unit of time of $\left(Y_{k h}, \sigma_{(k+1) h}^{2}\right)$ is

$$
\begin{aligned}
& \mathbb{E}\left[\frac{\left(\sigma_{(k+1) h}^{2}-\sigma_{k h}^{2}\right)^{2}}{h} \mid M_{k h}\right] \\
& =\frac{w^{(h)^{2}}}{h}+\sigma_{k h}^{2} \frac{2 w^{(h)}}{h}\left(\beta^{(h)}+\alpha^{(h)} \mathbb{E}\left[Z_{k h}^{2} \mid M_{k h}\right]-1\right)+\frac{\sigma_{k h}^{4}}{h} \mathbb{E}\left[\left(\beta^{(h)}+\alpha^{(h)} Z_{k h}^{2}-1\right)^{2} \mid M_{k h}\right] \\
& =\frac{w^{(h)^{2}}}{h}+\sigma_{k h}^{2} \frac{2 w^{(h)}}{h}\left(\beta^{(h)}+\alpha^{(h)}-1\right)+\frac{\sigma_{k h}^{4}}{h}\left(\left(\alpha^{(h)}+\beta^{(h)}-1\right)^{2}+\alpha^{(h)^{2}}\left(\mathbb{E}\left[Z_{k h}^{4} \mid M_{k h}\right]-1\right)\right) \\
& =\frac{w^{(h)^{2}}}{h}+\sigma_{k h}^{2} \frac{2 w^{(h)}}{h}\left(\beta^{(h)}+\alpha^{(h)}-1\right)+\frac{\sigma_{k h}^{4}}{h}\left(\alpha^{(h)}+\beta^{(h)}-1\right)^{2}+\frac{\sigma_{k h}^{4}}{h} 2 \alpha^{(h)^{2}}, \\
& \mathbb{E}\left[\frac{\left(Y_{k h}-Y_{(k-1) h}\right)^{2}}{h} \mid M_{k h}\right] \\
& =\left(\mu-\frac{\sigma_{k h}^{2}}{2}\right)^{2} h+\sigma_{k h}^{2} \mathbb{E}\left[Z_{k h}^{2} \mid M_{k h}\right]=\left(\mu-\frac{\sigma_{k h}^{2}}{2}\right)^{2} h+\sigma_{k h}^{2}, \\
& \mathbb{E}\left[\frac{\left(Y_{k h}-Y_{(k-1) h}\right)\left(\sigma_{(k+1) h}^{2}-\sigma_{k h}^{2}\right)}{h} \mid M_{k h}\right] \\
& =\mathbb{E}\left[\frac{\left(\left(\mu-\frac{\sigma_{k h}^{2}}{2}\right) h+\sigma_{k h} \sqrt{h} Z_{k h}\right)\left(w^{(h)}+\sigma_{k h}^{2}\left(\beta^{(h)}+\alpha^{(h)} Z_{k h}^{2}-1\right)\right)}{h} \mid M_{k h}\right] \\
& =\left(\mu-\frac{\sigma_{k h}^{2}}{2}\right) w^{(h)}+\sigma_{k h}^{2}\left(\mu-\frac{\sigma_{k h}^{2}}{2}\right)\left(\beta^{(h)}+\alpha^{(h)} \mathbb{E}\left[Z_{k h}^{2} \mid M_{k h}\right]-1\right) \\
& \left.+\frac{\sigma_{k h}}{\sqrt{h}} \mathbb{E}\left[Z_{k h} w^{(h)} \sigma_{k h}^{2}\left(\beta^{(h)} Z_{k h}+\alpha^{(h)} Z_{k h}^{3}-Z_{k h}\right)\right) \mid M_{k h}\right] \\
& =\left(\mu-\frac{\sigma_{k h}^{2}}{2}\right) w^{(h)}+\sigma_{k h}^{2}\left(\mu-\frac{\sigma_{k h}^{2}}{2}\right)\left(\beta^{(h)}+\alpha^{(h)}-1\right) .
\end{aligned}
$$

Such a second moment has to converge to the variance-covariance matrix per unit of time of the

GARCH diffusion model,

$$
\frac{\operatorname{Var}\left(\left(\log S_{t+d t}, V_{t+d t}\right) \mid \mathcal{F}_{t}\right)}{d t}=\left[\begin{array}{cc}
V_{t} & 0 \\
0 & c_{3}^{2} V_{t}^{2}
\end{array}\right] .
$$

This implies that

$$
\lim _{h \downarrow 0} \frac{2 \alpha^{(h)^{2}}}{h}=c_{3}^{2},
$$


giving the relationship between $\alpha^{(h)}$ and $c_{3}$. Substituting (A.36), ( A.37) and (A.41) into (A.38), (A.39) and(A.40), one has

$$
\begin{aligned}
& \mathbb{E}\left[\frac{\left(\sigma_{(k+1) h}^{2}-\sigma_{k h}^{2}\right)^{2}}{h} \mid M_{k h}\right]=\alpha^{(h)^{2}} \sigma_{k h}^{4}+o(1) \\
& \mathbb{E}\left[\frac{\left(Y_{k h}-Y_{(k-1) h}\right)^{2}}{h} \mid M_{k h}\right]=\sigma_{k h}^{2}+o(1) \\
& \mathbb{E}\left[\frac{\left(Y_{k h}-Y_{(k-1) h}\right)\left(\sigma_{(k+1) h}^{2}-\sigma_{k h}^{2}\right)}{h} \mid M_{k h}\right]=o(1)
\end{aligned}
$$

where the $o(1)$ term vanish uniformly on compact sets as $h$ goes to zero. Up to order $o(h)$, the sequence $\left\{w^{(h)}, \beta^{(h)}, \alpha^{(h)}\right\}$ is

$$
c_{1}=w^{(h)} / h, \quad c_{2}=1-\beta^{(h)}-\alpha^{(h)}, \quad c_{3}=\alpha^{(h)} \sqrt{2 / h} .
$$

Under such a sequence, the fourth moments of $\left(Y_{k h}-Y_{(k-1) h}\right)$ and $\left(\sigma_{(k+1) h}^{2}-\sigma_{k h}^{2}\right)$ converge to zero and the convergence result holds. 


\section{Bibliography}

[1] Andersen T.G., L. Benzoni and J. Lund (2002), "An empirical investigation of continuous-time equity retur models", Journal of Finance, 57, 1239-1284.

[2] Ball C. A. and A. Roma (1994), "Stochastic Volatility Option Pricing", Journal of Financial and Quantitative Analysis, 29, 589-607.

[3] Barone-Adesi G., H. Rasmussen and C. Ravanelli (2003), "An Option Pricing Formula for the GARCH Diffusion Model", working paper, University of Lugano and NCCR FINRSIK.

[4] Barone-Adesi G. and R. E. Whaley (1987), "Efficient analytic approximation of American option values", Journal of Finance, 42, 1-20.

[5] Beckers S.(1981), "Standard Deviations Implied in Option Process as Predictors of Future Stock Price Variability", Journal of Banking and Finance, 5, 363-382.

[6] Black F. and M. Scholes (1973), "The Pricing of Options and Corporate Liabilities", Journal of Political Economy, 81, 637-659.

[7] Bhattacharya R. N. and E. C. Waymire (1990), Stochastic Processes with Application, John Wiley and son, New York, N.Y. 
[8] Bodurtha J. and G. Courtadon (1987), "Tests of the American Option Pricing Model in the Foreign Currency Option Market", Journal of Financial and Quantitative Analysis, 22, 153-167.

[9] Bollerslev T. and H. Zhou (2002), "Estimating Stochastic Volatility Diffusion using Conditional Moments of Integrated Volatility", Journal of Econometrics, 109, 33-65.

[10] Boyle P., M. Broadie and P. Glasserman (1997), "Monte Carlo Methods for Security Pricing", Journal of Economic Dynamics and Control, 21, 1267-1321.

[11] Breeden D. T. (1979), "An Intertemporal Asset Pricing Model with Stochastic Consumption and Investment Opportunities", Journal of Financial Economics, 7, 265-296.

[12] Canina L. and Figlewskki(1993), "The Informational Content of Implied Volatility", Rewiev of Financial Studies, 6, 659-681.

[13] Chernov M. and E. Ghysels (2000), "A Study Towards a Unified Approach to the Joint Estimation of Objective and Risk-Neutral Measures for the Purpose of Options Valuation", Journal of Financial Economics, 56, 407-458.

[14] Chesney M. and L.O. Scott (1989), "Pricing European Currency Options: a Comparison of the Modified Black-Scholes Model and a Random Variance Model", Journal of Financial and Quantitative Analysis, 24, 267-284.

[15] Cont R. (2001), "Empirical properties of asset returns: stylized facts and statistical issues", Quantitative Finance, 1, 223-236.

[16] Cox J. C., J. E. Ingersoll and S. A. Ross (1995), "A Theory of the Term Structure of Interest Rates", Econometrica, 53, 385-408.

[17] Cox D. R. and H. D. Miller (1972), The Theory of Stochastic Processes, Chapman and Hall, London. 
[18] Dacorogna M. M, R. Genay, U. A. Mller, R. B. Olsen and O.V. Pictet (2001), TAn Introduction to High-Frequency Finance, Academic Press, San Diego, CA.

[19] Delbaen, F., and W. Schachermayer (1994), "A General Version of the fundamental Theorem of Asset pricing", Mathematische Annalen, 300, 463-520.

[20] Engle R.F. and T. Bollerslev (1986), "Modelling the Persistence of Conditional Variances", Econometric Reviews, 5, 1-50.

[21] Engle R.F. and G.G.J. Lee (1996), "Estimating Diffusion Models of Stochastic Volatility", in Rossi P.E. (Ed.), Modeling Stock Market Volatility: Bridging the Gap to Continuous Time, New York: Academic Press, 333-384.

[22] Fama E. (1965), "The Behaviour of Stock Prices", Journal of Business, 38, 34-105.

[23] Fouque J., G. Papanicolaou and K.R. Sircar (2000), Derivatives in Financial Markets with Stochastic Volatility, Cambridge University Press, Cambridge.

[24] Garcia R., M.A. Lewis and E. Renault (2001), "Estimation of Objective and Risk-Neutral Distributions Based on Moments of the Integrated Volatility ", working paper, Cirano.

[25] Genon-Catalot V., T. Jeantheau and C. Laredo (2000), "Stochastic Volatility Models as Hidden Markov Models and Statistical Applications", Bernoulli, 6, 1051-1079.

[26] Gesser V. and P. Poncet (1997), "Volatility Patterns: Theory and Some Evidence from the DollarMark Option Market", The Journal of Derivatives, 5, 46-65.

[27] Guo D. (1996), "The Predictive Power of Implied Stochastic Variance from Currency Options", Journal of Futures Markets, 16, 915-942. 
[28] Guo D. (1998), "The Risk Premium of Volatility Implicit in Currency Options", Journal of Business and Economic Statistics, 16, 498-507.

[29] Gourieroux C., A. Monfort and E. Renault(1993), "Inderect Inference", Journal of Applied Econometrics, 8, Suppl. and S85-S118.

[30] Harrison J.M. and S. Pliska (1981), "Martingales and Stochastic Integrals in the Theory of Continuous trading", Stochastic Processes and their Applications, 11, 215-260.

[31] Harrison J.M. and S. Pliska (1933), "A Stochastic Calculus Model of Continuous Trading: Complete Markets", Stochastic Processes and their Applications, 15, 313-316.

[32] Heston S. (1993), "A Closed-Form Solution for Options with Stochastic Volatility with Applications to Bond and Currency Options", Review of Financial Studies, 6, 327-343.

[33] Hull J. and A. White (1987), "The Pricing of Options on Assets with Stochastic Volatilities", Journal of Finance, 42, 281-300.

[34] Hull J. and A. White (1987a), "Hedging the Risks from Writing Foreign Currency Options", Journal of International money and Finance, 42, 131-152.

[35] Hull J. and A. White (1988), "An Analysis of the Bias in Option Pricing Caused by a Stochastic Volatility", Journal of International Economics, 24, 129-145.

[36] Jiang G. (1998), "Stochastic Volatility and Option Pricing", in J.L. Knight and S. E. Satchell (Eds.) Forecasting Volatility in the Financial Market, London: Butterworth Hernemann, pp. 4596.

[37] Karatzas I. and S. Shreve (1991), Brownian Motion and Stochastic Calculus, Springer-Verlag, New York. 
[38] Kloeden P.E. and E. Platen (1999), Numerical Solution of Stochastic Differential Equations, Springer-Verlag, New York.

[39] Jorion P. (1995), "Predicting Volatility in the Foreign Exchange Market", Journal of Finance, 50, 507-528.

[40] Jones C.S. (2003), "The Dynamics of Stochastic Volatility: Evidence from Underlying and Options Markets", Journal of Econometrics, 116, 181-224.

[41] Lantane H. and R. Rendleman (1976), "Standard Deviation of Stock Price Ratios Implied in Option Prices" Journal of Finance, 31, 369-381.

[42] Leippold M., Trojani F. and Vanini P. "A Geometric Approach to Multiperiod Mean-Variance Optimization of Assets and Liabilities" Journal of Economic Dynamics and Control, forthcoming.

[43] Lewis A.L. (2000), Option Valuation under Stochastic Volatility, Finance Press, California, USA.

[44] Lewis A.L. (2000a), "Analytical Expressions for the Moments of the Integrated Volatility in Affine Stochastic Volatility Models", working paper, Cirano.

[45] Mandelbrot B. (1963), "The Variation of Certain Speculative Prices", Journal of Business, 36, 394-419.

[46] Melenberg B. and B.J.M. Werker (2001), "The Pricing of Volatility Risk: An Empirical Example", working paper, Tilburg University.

[47] Melino A. and S.M. Turnbull (1990), "Pricing Foreign Currency Options with Stochastic Volatility", Journal of Econometrics, 45, 239-265.

[48] Merton R.C. (1973), "Theory of Rational Option Pricing", Bell Journal of Economics and Management Science, 4, 141-183. 
[49] Nelson D.B. (1990), "ARCH Models as Diffusion Approximations", Journal of Econometrics, 45, 7-38.

[50] Renault E. and N. Touzi (1996), "Option Hedging and Implied Volatilities in a Stochastic Volatility Model", Mathematical Finance, 6, 279-302.

[51] Sircar K.R. and G.C. Papanicolaou (1999), "Stochastic Volatility, Smile and Asymptotics", Applied Mathematical Finance, 6, 107-145.

[52] Scott L.O. (1987), "Option Pricing when the Variance Change Randomly: Theory, Estimation and an Application", Journal of Financial and Quantitative Analysis, 22, 419-438.

[53] Taylor S.J., (1994), "Modeling Stochastic Volatility: a Review and Comparative Study", Mathematical Finance 4, 183-204.

[54] Taylor S.J. and X. Xu (1994), "The Magnitude of Implied Volatility Smiles: Theory and Empirical Evidence for Exchange Rates", The Review of Futures Markets, 13, 355-380.

[55] Wiggins J. (1987), "Option Values under Stochastic Volatility", Journal of Financial Economics, 19, 351-372.

[56] Wong E. (1964), "The Construction of a Class of Stationary Markoff Processes", in Belleman R. (Ed.), Sixteenth Symposium in Applied Mathematics, Stochastic Processes in Mathematical Physics and Engineering, American Mathematical Society, Providence, 264-276.

[57] Xu X. and S.J. Taylor (1994), "Term Structure of Volatility Implied by Foreign Exchange Options", Journal of Finance and Quantitative Analysis, 29, 57-74. 